\title{
Synthesis of Dihydrophenanthridines and Oxoimidazolidines from Anilines and Ethylglyoxylate via Aza Diels-Alder Reaction of Arynes and KF-Induced Annulation
}

R. Santhosh Reddy, ${ }^{\dagger}$ Chandraiah Lagishetti, ${ }^{\dagger}$ Shuo Chen, I. N. Chaithanya Kiran and Yun $\mathrm{He}^{*}$

$\underline{\text { S.No }}$ Table of Contents $\quad \underline{\text { Page }}$

1 General information $\quad$ S2

2 Literature reports for aryne ADAR and annulation of ethyl (arylimino) acetates

3 General procedure for the optimization of the reaction conditions: ADAR S4

4 General procedure for the ADAR of MCR involving anilines, ethylglyoxylate and arynes

$5 \quad$ Procedure for ADAR with isolated imine $6 \mathbf{a}$

6 General procedure for the optimization of the reaction conditions for oxoimidazolidine derivatives 7

$7 \quad$ General procedure for the synthesis of oxoimidazolidine derivatives 7

8 References 


\section{General Information}

Unless otherwise specified, all reactions were carried out under an atmosphere of argon in flame-dried reaction vessels with Teflon screw caps. Reaction temperatures are reported as the temperature of the bath surrounding the reaction vessel. $25{ }^{\circ} \mathrm{C}$ corresponds to the room temperature of the lab when the experiments were carried out. THF and MeCN were freshly purified by distillation over Na-benzophenone and calcium hydride respectively. 18-Crown-6 was recrystallized from dry $\mathrm{MeCN}$ and $\mathrm{KF}$ was dried by heating at $110{ }^{\circ} \mathrm{C}$ for $12 \mathrm{~h}$ and left to cool under argon. Reactions were monitored by analytical thin-layer chromatography (TLC) on Merck silica gel $60 \mathrm{~F}_{254}$ plates $(0.25 \mathrm{~mm})$, visualized by ultraviolet light. ${ }^{1} \mathrm{H}$ NMR spectra were obtained on an Agilent 400MR or 600MR DD2 spectrometer at ambient temperature.

Data were reported as follows: chemical shift on the $\delta$ scale using residual proton solvent as internal standard [ठ 0.00 (TMS)], multiplicity $(\mathrm{br}=$ broad, $\mathrm{s}=$ singlet, $\mathrm{d}=$ doublet, $\mathrm{t}=$ triplet, $\mathrm{q}=$ quartet, $\mathrm{m}=$ multiplet), integration, and coupling constant(s) in hertz. ${ }^{13} \mathrm{C} \mathrm{NMR}$ spectra were obtained with proton decoupling on an Agilent 400MR DD2 (101 MHz) spectrometer and were reported in ppm with residual solvent for internal standard $[\delta 77.0$ $\left.\left(\mathrm{CDCl}_{3}\right)\right]$. High resolution mass spectra were obtained on a Bruker SolariX 7.0T spectrometer. Melting point was determined by WRS-2A Digital Melting Point Apparatus. IR spectra were recorded on a Bruker $100 \mathrm{FT}-\mathrm{IR}$ spectrometer and are reported in terms of frequency of absorption $\left(\mathrm{cm}^{-1}\right)$. The 2-(trimethylsilyl)phenyl trifluoromethane sulfonate $\mathbf{3 a}$ and the other symmetric and unsymmetric aryne precursors were synthesized following literature procedure. ${ }^{1}$ 


\section{Literature reports for aryne ADAR and annulation of ethyl (arylimino)acetates}

Previous work

(a) Wang, et. al. ${ }^{2 a} \mathrm{~J}$. Org. Chem. 2006, 71, 9241.<smiles>Oc1ccc(N=CBr)cc1</smiles>

electrin-rich anilines \&

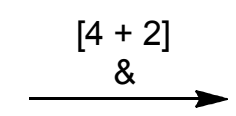

aromatization

normal electron-demand

benzaldehyde derivatives

(b) Coquerel/ Rodriguez, et. al. ${ }^{2 b, c}$ Org. Lett. 2015, 17, 3374

and J. Org. Chem. 2015, 80, 9767.<smiles>Cc1cc(N=C[Al-])n(C)n1</smiles>

electron-rich $\mathrm{N}$-heteroaryl amines

\& benzaldehyde derivatives

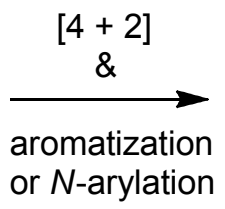

normal electron-demand

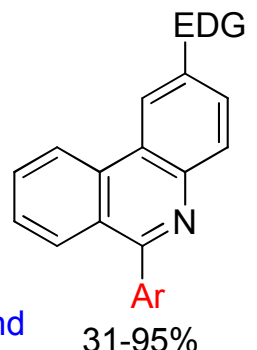

$31-95 \%$

(c) Shi, et. al. ${ }^{3}$ Chem. Commun. 2008, 4998.

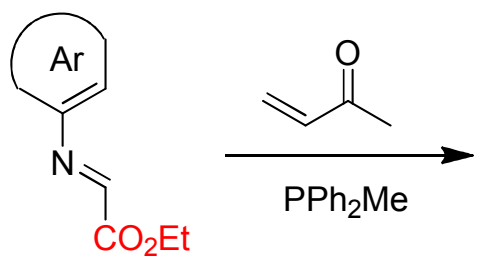<smiles>CCOCC1N([Al])C(=O)C(N)N1[Al]</smiles>

This work<smiles>CCOC(=O)C1c2ccccc2-c2ccccc2N1c1ccccc1</smiles>
inverse electron-demand<smiles>CCOC(=O)/C=N/c1ccccc1</smiles>
electron-poor imines<smiles></smiles>

$\mathrm{R}=$ : or $\mathrm{H}$ or $\mathrm{Ph}$ $30-93 \%$ 


\section{General procedure for the optimization of the reaction conditions: ADAR}

Table S1: Optimization of Reaction Conditions ${ }^{a}$

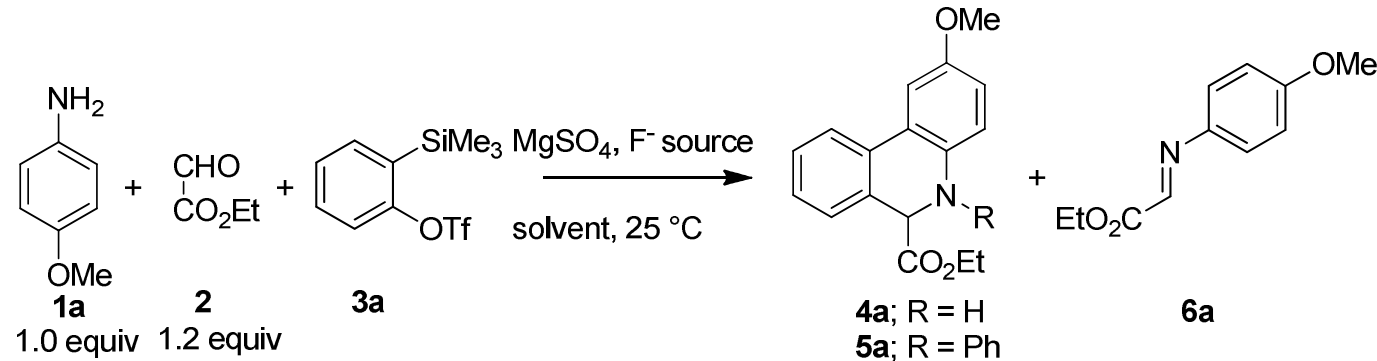

\begin{tabular}{|c|c|c|c|c|c|c|}
\hline \multirow{2}{*}{ entry } & \multirow{2}{*}{$\begin{array}{c}3 \mathbf{a} \\
{[\text { equiv] }}\end{array}$} & \multirow{2}{*}{$\begin{array}{c}\mathrm{F}^{-} \text {source } \\
\text { [equiv] }\end{array}$} & \multirow{2}{*}{$\begin{array}{c}\text { additive } \\
\text { [equiv] }\end{array}$} & \multirow{2}{*}{ solvent } & \multicolumn{2}{|c|}{ yield [\%] } \\
\hline & & & & & $5 a$ & $6 a$ \\
\hline 1 & 1.2 & $\mathrm{KF}(2.5)$ & $18-c-6(2.5)$ & $\mathrm{MeCN}$ & 42 & $38(9)^{c}$ \\
\hline 2 & 1.2 & $\operatorname{CsF}(2.5)$ & - & $\mathrm{MeCN}$ & 36 & $40(9)^{\mathrm{c}}$ \\
\hline 3 & 1.2 & $K F(2.5)^{d}$ & $18-c-6(2.5)$ & THF & 38 & $40(12)^{c}$ \\
\hline 4 & 1.2 & $\mathrm{KF}(2.5)^{\mathrm{d}, \mathrm{e}}$ & $18-c-6(2.5)$ & $\mathrm{MeCN}$ & 38 & $36(9)^{c}$ \\
\hline 5 & 1.2 & $\mathrm{KF}(2.5)^{f}$ & $18-c-6(2.5)$ & $\mathrm{MeCN}$ & 40 & $41(12)^{\mathrm{C}}$ \\
\hline 6 & 2.0 & $\mathrm{KF}(4.0)^{\mathrm{e}}$ & $18-c-6(4.0)$ & THF & 48 & $32(12)^{c}$ \\
\hline 7 & 3.0 & $\mathrm{KF}(5.0)^{\mathrm{e}}$ & $18-c-6(5.0)$ & THF & 57 & $28(12)^{\mathrm{C}}$ \\
\hline 8 & 3.0 & $\mathrm{KF}(5.0)$ & $18-c-6(5.0)$ & $\mathrm{MeCN}$ & 63 & $24(10)^{\mathrm{C}}$ \\
\hline 9 & 3.0 & $\mathrm{KF}(5.0)$ & $18-c-6(5.0)$ & $\begin{array}{l}\text { MeCN: } \\
\text { PhMe }^{\mathrm{g}}\end{array}$ & 88 & $4(4)^{\mathrm{c}}$ \\
\hline 10 & 3.0 & TBAT & - & $\begin{array}{l}\text { MeCN: } \\
\text { PhMe }^{g}\end{array}$ & $35^{\mathrm{h}}$ & $22(18)^{c}$ \\
\hline 11 & 3.0 & $\mathrm{CsF}$ & - & $\begin{array}{l}\text { MeCN: } \\
\text { PhMe }^{g}\end{array}$ & $38^{h}$ & $25(20)^{\mathrm{C}}$ \\
\hline 12 & 3.0 & TBAF & - & $\begin{array}{l}\text { MeCN: } \\
\text { PhMe }^{\mathrm{g}}\end{array}$ & $<8$ & $49(18)^{c}$ \\
\hline
\end{tabular}

${ }^{\mathrm{a}}$ Standard conditions: $1 \mathrm{a}(0.20 \mathrm{mmol}), 2$ (0.24 mmol), $\mathrm{MgSO}_{4}(3.0$ equiv), solvent $(2.0 \mathrm{~mL}), 1 \mathrm{~h}$ then fluoride source, $3 \mathrm{a}$, rt, $2 \mathrm{~h}$. ${ }^{\mathrm{b}}$ Isolated yields. ${ }^{\mathrm{c}}\left(4-\mathrm{OMeC}_{6} \mathrm{H}_{4}\right) \mathrm{PhNH}$. ${ }^{\mathrm{d}} \mathrm{MnO}_{2}$ (10.0 equiv). ${ }^{\mathrm{e}}$ Reaction temperature $70{ }^{\circ} \mathrm{C} .{ }^{f} \mathrm{O}_{2}$ (balloon). ${ }^{9} \mathrm{MeCN}$ :PhMe (1:1). ${ }^{\mathrm{h}}$ Reaction time $24 \mathrm{~h}$. 
General procedure: To a flame-dried screw-capped test tube equipped with a magnetic stir bar was added the aniline (1a, $0.2 \mathrm{mmol})$, ethylglyoxylate (2, 50\% in $\mathrm{PhMe}, 0.24 \mathrm{mmol})$ and $\mathrm{MgSO}_{4}$ (3 equiv) under argon atmosphere. The mixture was dissolved in $2.0 \mathrm{~mL}$ of solvent under argon atmosphere. The resultant reaction mixture was kept in stirring for $1 \mathrm{~h}$ for the imine formation. To the resultant solution was then added fluoride source and the aryne precursor $3 \mathbf{a}$. Then the reaction mixture was stirred at $25{ }^{\circ} \mathrm{C}$ till the completion of reaction (monitored by TLC). The reaction mixture was diluted with EtOAc $(2.0 \mathrm{~mL})$ and filtered through short pad of silica gel and eluted with EtOAc $(10 \mathrm{~mL})$. The solvent was evaporated to obtain the crude product, which was purified by flash column chromatography on silica gel.

\section{General procedure for the ADAR of MCR involving anilines, ethylglyoxylate and arynes}

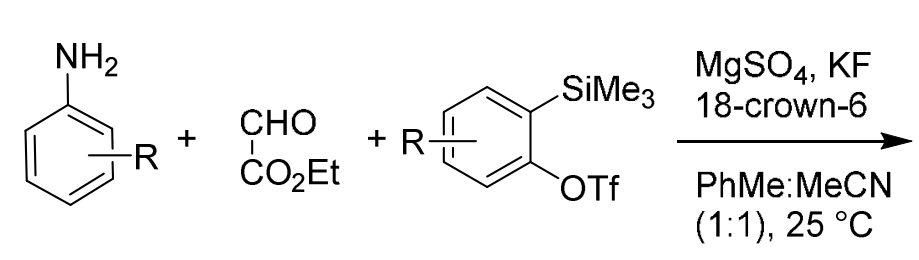

1
2
3

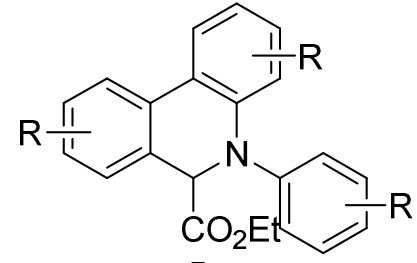

5

To a flame-dried screw-capped test tube equipped with a magnetic stir bar was added the aniline (1a, $0.2 \mathrm{mmol}$ ), ethylglyoxylate (2, 50\% in $\mathrm{PhMe}, 0.24 \mathrm{mmol}$ ) and $\mathrm{MgSO}_{4}$ (3 equiv) under argon atmosphere. The mixture was dissolved in $2.0 \mathrm{~mL}$ of PhMe:MeCN (1:1) under argon atmosphere. The resultant reaction mixture was kept in stirring for $1 \mathrm{~h}$ for the imine formation. To the resultant solution was then added 18-crown-6 $(1.0 \mathrm{mmol}), \mathrm{KF}(1.0 \mathrm{mmol})$ and the aryne precursor $3 a(0.6 \mathrm{mmol})$. Then the reaction mixture was stirred at $25^{\circ} \mathrm{C}$ till the completion of reaction (monitored by TLC; typically after $2 \mathrm{~h}$ ). Then the reaction mixture was diluted with EtOAc $(2.0 \mathrm{~mL})$ and filtered through short pad of silica gel and eluted with EtOAc $(10 \mathrm{~mL})$. The solvent was evaporated to obtain the crude product, which was purified by flash column chromatography on silica gel to afford the corresponding $\mathrm{N}$-aryl dihydrophenanthridine carboxylate derivatives 5 a-s in moderate to high yields. 


\section{Ethyl 2-methoxy-5-phenyl-5,6-dihydrophenanthridine-6-carboxylate (5a)}

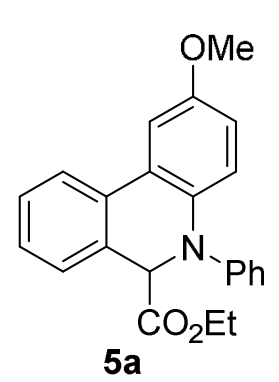

Pale yellow oil $\left(0.063 \mathrm{~g}, 88 \%\right.$ yield); $\mathrm{R}_{f}=0.4$ (silica, EtOAc:hexanes, 1:9); IR

(film) $\mathrm{v}_{\max } 2927,1739,1496,1223,1021,764 \mathrm{~cm}^{-1} ;{ }^{1} \mathrm{H}$ NMR $(400 \mathrm{MHz}$, $\left.\mathrm{CDCl}_{3}\right) \delta=7.80(\mathrm{~d}, J=7.8 \mathrm{~Hz}, 1 \mathrm{H}), 7.42(\mathrm{td}, J=7.5,1.6 \mathrm{~Hz}, 1 \mathrm{H}), 7.38-7.30$ $(\mathrm{m}, 5 \mathrm{H}), 7.25-7.17(\mathrm{~m}, 3 \mathrm{H}), 7.03(\mathrm{td}, J=7.3,1.3 \mathrm{~Hz}, 1 \mathrm{H}), 6.81(\mathrm{dd}, J=9.0$, $3.0 \mathrm{~Hz}, 1 \mathrm{H}), 5.43(\mathrm{~s}, 1 \mathrm{H}), 4.20-4.02(\mathrm{~m}, 2 \mathrm{H}), 3.87(\mathrm{~s}, 3 \mathrm{H}), 1.15(\mathrm{t}, J=7.3$ $\mathrm{Hz}, 3 \mathrm{H}) ;{ }^{13} \mathrm{C}$ NMR $\left(101 \mathrm{MHz}, \mathrm{CDCl}_{3}\right) \delta=171.4,154.1,146.4,135.3,131.5,130.7,129.1,128.4$, $127.6,127.3,125.4,122.8,122.1,120.9,120.0,114.8,108.6,64.3,61.2,55.5,14.0$ ppm; HRMS (m/z): [M + Na] $]^{+}$calcd for $\mathrm{C}_{23} \mathrm{H}_{21} \mathrm{NO}_{3} \mathrm{Na}^{+}$382.1413, Found 382.1414.

\section{Ethyl 5-phenyl-5,6-dihydrophenanthridine-6-carboxylate (5b)}

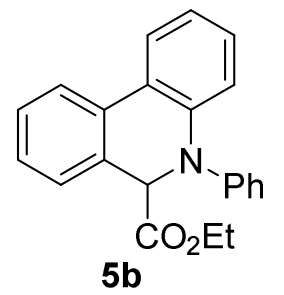

Pale yellow oil $\left(0.054 \mathrm{~g}, 82 \%\right.$ yield); $\mathrm{R}_{f}=0.5$ (silica, EtOAc:hexanes, 1:9); IR (film) $V_{\max } 3012,1738,1502,1380,1012,765 \mathrm{~cm}^{-1} ;{ }^{1} \mathrm{H}$ NMR $(400 \mathrm{MHz}$, $\left.\mathrm{CDCl}_{3}\right) \delta=7.80(\mathrm{dd}, J=16.5,7.8 \mathrm{~Hz}, 2 \mathrm{H}), 7.41(\mathrm{t}, J=7.6 \mathrm{~Hz}, 1 \mathrm{H}), 7.38-$ $7.29(\mathrm{~m}, 4 \mathrm{H}), 7.28-7.24(\mathrm{~m}, 2 \mathrm{H}), 7.17-7.14(\mathrm{~m}, 2 \mathrm{H}), 7.12-7.06(\mathrm{~m}, 1 \mathrm{H})$, $6.99-6.96(\mathrm{~m}, 1 \mathrm{H}), 5.40(\mathrm{~s}, 1 \mathrm{H}), 4.19-4.00(\mathrm{~m}, 2 \mathrm{H}), 1.12(\mathrm{t}, J=7.1 \mathrm{~Hz}, 3 \mathrm{H}) ;{ }^{13} \mathrm{C}$ NMR $(101$ $\left.\mathrm{MHz}, \mathrm{CDCl}_{3}\right) \delta=171.4,145.9,142.1,130.9,130.9,129.2,128.6,128.5,127.5,127.1,123.9$ 123.7, 123.2, 122.7, 122.2, 120.5, 118.0, 64.6, 61.3, 13.9 ppm; HRMS (m/z): $[M+H]^{+}$calcd for $\mathrm{C}_{22} \mathrm{H}_{20} \mathrm{NO}_{2} 330.1488$, Found 330.1486.

\section{Ethyl 2-ethoxy-5-phenyl-5,6-dihydrophenanthridine-6-carboxylate (5c)}<smiles>CCOc1ccc2c(c1)-c1ccccc1C(C(F)(F)F)N2c1ccccc1</smiles>

Pale yellow oil (0.066 g, 88\% yield); $\mathrm{R}_{f}=0.4$ (silica, EtOAc:hexanes, 1:9); IR (film) $v_{\max } 2980,1741,1496,1200,1048,764 \mathrm{~cm}^{-1} ;{ }^{1} \mathrm{H}$ NMR $(400 \mathrm{MHz}$, $\left.\mathrm{CDCl}_{3}\right) \delta=7.80(\mathrm{~d}, J=7.8 \mathrm{~Hz}, 1 \mathrm{H}), 7.42(\mathrm{t}, J=7.5 \mathrm{~Hz}, 1 \mathrm{H}), 7.39-7.28(\mathrm{~m}$, $5 \mathrm{H}), 7.25-7.16(\mathrm{~m}, 3 \mathrm{H}), 7.03(\mathrm{t}, J=7.4 \mathrm{~Hz}, 1 \mathrm{H}), 6.80(\mathrm{dd}, J=8.9,2.8 \mathrm{~Hz}$, 
1H), $5.43(\mathrm{~s}, 1 \mathrm{H}), 4.20-4.02(\mathrm{~m}, 4 \mathrm{H}), 1.46(\mathrm{t}, J=7.0 \mathrm{~Hz}, 3 \mathrm{H}), 1.15(\mathrm{t}, J=7.1 \mathrm{~Hz}, 3 \mathrm{H}) ;{ }^{13} \mathrm{C} \mathrm{NMR}$ $\left(101 \mathrm{MHz}, \mathrm{CDCl}_{3}\right) \delta=171.4,153.4,146.4,135.2,131.5,130.8,129.1,128.4,127.6,127.2$, $125.4,122.8,122.1,120.8,119.9,115.4,109.4,64.4,63.8,61.2,14.9,13.9$ ppm; HRMS (m/z): $[\mathrm{M}+\mathrm{Na}]^{+}$calcd for $\mathrm{C}_{24} \mathrm{H}_{23} \mathrm{NO}_{3} \mathrm{Na}^{+} 396.1570$, Found 396.1566.

\section{Ethyl 2-methyl-5-phenyl-5,6-dihydrophenanthridine-6-carboxylate (5d)}

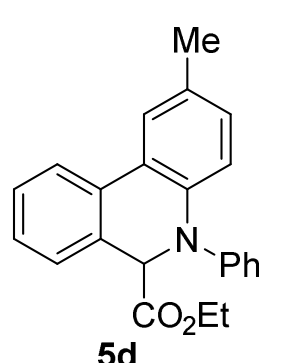

$5 d$

Pale yellow oil $\left(0.058 \mathrm{~g}, 86 \%\right.$ yield); $\mathrm{R}_{f}=0.5$ (silica, EtOAc:hexanes, $\left.1: 9\right)$; IR (film) $V_{\max } 2924,1744,1497,1023,695 \mathrm{~cm}^{-1} ;{ }^{1} \mathrm{H}$ NMR $\left(400 \mathrm{MHz}, \mathrm{CDCl}_{3}\right)$ $\delta=7.83(\mathrm{~d}, J=7.8 \mathrm{~Hz}, 1 \mathrm{H}), 7.61(\mathrm{~d}, J=2.1 \mathrm{~Hz}, 1 \mathrm{H}), 7.41(\mathrm{t}, J=7.5 \mathrm{~Hz}$,

$1 \mathrm{H}), 7.38-7.29(\mathrm{~m}, 4 \mathrm{H}), 7.27-7.23(\mathrm{~m}, 2 \mathrm{H}), 7.13(\mathrm{dd}, J=8.3,1.4 \mathrm{~Hz}$, 1H), $7.06(\mathrm{t}, J=7.3 \mathrm{~Hz}, 1 \mathrm{H}), 7.00(\mathrm{dd}, J=8.4,2.0 \mathrm{~Hz}, 1 \mathrm{H}), 5.41(\mathrm{~s}, 1 \mathrm{H})$, $4.20-4.01(\mathrm{~m}, 2 \mathrm{H}), 2.38(\mathrm{~s}, 3 \mathrm{H}), 1.15(\mathrm{t}, \mathrm{J}=7.1 \mathrm{~Hz}, 3 \mathrm{H}) ;{ }^{13} \mathrm{C}$ NMR $\left(101 \mathrm{MHz}, \mathrm{CDCl}_{3}\right) \delta=$ $171.4,146.1,139.4,131.1,130.9,129.8,129.4,129.1,128.4,127.5,127.0,124.2,124.0,122.7$ 122.6, 121.6, 118.2, 64.4, 61.2, 20.9, 14.0 ppm; HRMS (m/z): $[\mathrm{M}+\mathrm{H}]^{+}$calcd for $\mathrm{C}_{23} \mathrm{H}_{22} \mathrm{NO}_{2}$ 344.1645, Found 344.1642.

\section{Ethyl 2-ethyl-5-phenyl-5,6-dihydrophenanthridine-6-carboxylate (5e)}<smiles>CCOC(=O)C1c2ccccc2-c2cc(CC)ccc2N1c1ccccc1</smiles>

$\mathrm{Hz}, 1 \mathrm{H}), 7.10-7.01(\mathrm{~m}, 2 \mathrm{H}), 5.42(\mathrm{~s}, 1 \mathrm{H}), 4.20-4.02(\mathrm{~m}, 2 \mathrm{H}), 2.69(\mathrm{q}, J=$

$7.6 \mathrm{~Hz}, 2 \mathrm{H}), 1.31(\mathrm{t}, J=7.6 \mathrm{~Hz}, 3 \mathrm{H}), 1.15(\mathrm{t}, J=7.1 \mathrm{~Hz}, 3 \mathrm{H}) ;{ }^{13} \mathrm{C} \operatorname{NMR}\left(101 \mathrm{MHz}, \mathrm{CDCl}_{3}\right) \delta=$ $171.5,146.1,139.6,136.3,131.1,131.0,129.1,128.4,128.2,127.5,126.9,123.9,122.9,122.7$, 122.6, 121.6, 118.3, 64.5, 61.2, 28.3, 15.6, 13.9 ppm; HRMS (m/z): $\left[\mathrm{M}+\mathrm{Na}^{+}\right.$calcd for $\mathrm{C}_{24} \mathrm{H}_{23} \mathrm{NO}_{2} \mathrm{Na}^{+} 380.1621$, Found 380.1619. 
Ethyl 2-isopropyl-5-phenyl-5,6-dihydrophenanthridine-6-carboxylate (5f)<smiles>CCOC(=O)C1c2ccccc2-c2cc(C(C)C)ccc2N1c1ccccc1</smiles>

$(\mathrm{m}, 1 \mathrm{H}), 1.30(\mathrm{~s}, 3 \mathrm{H}), 1.29(\mathrm{~s}, 3 \mathrm{H}), 1.12(\mathrm{t}, J=7.1 \mathrm{~Hz}, 3 \mathrm{H}) ;{ }^{13} \mathrm{C}$ NMR $\left(101 \mathrm{MHz}, \mathrm{CDCl}_{3}\right) \delta=$ $171.5,146.1,140.9,139.7,131.2,131.1,129.1,128.4,127.6,126.9,126.8,123.8,122.7,122.6$ 121.6, 121.5, 118.2, 64.5, 61.2, 33.6, 24.1, 24.0, 13.9 ppm; HRMS (m/z): $[\mathrm{M}+\mathrm{Na}]^{+}$calcd for $\mathrm{C}_{25} \mathrm{H}_{25} \mathrm{NO}_{2} \mathrm{Na}^{+}$394.1777, Found 394.1776.

\section{Ethyl 4-methyl-5-phenyl-5,6-dihydrophenanthridine-6-carboxylate (5g)}

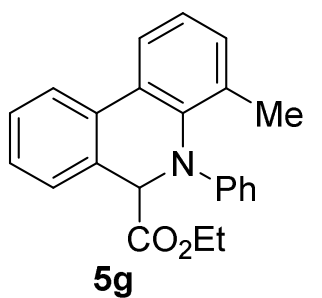

Pale yellow oil $\left(0.057 \mathrm{~g}, 83 \%\right.$ yield); $\mathrm{R}_{f}=0.5$ (silica, EtOAc:hexanes, 1:9); IR (film) $v_{\max } 2963,1744,1597,1495,1024,764 \mathrm{~cm}^{-1} ;{ }^{1} \mathrm{H}$ NMR $\left(400 \mathrm{MHz}, \mathrm{CDCl}_{3}\right) \delta=7.85(\mathrm{~d}, J=7.9 \mathrm{~Hz}, 1 \mathrm{H}), 7.74-7.69(\mathrm{~m}, 1 \mathrm{H}), 7.40$ $-7.36(\mathrm{~m}, 1 \mathrm{H}), 7.31-7.27(\mathrm{~m}, 2 \mathrm{H}), 7.23-7.17(\mathrm{~m}, 2 \mathrm{H}), 7.15(\mathrm{~d}, J=4.7$

$\mathrm{Hz}, 2 \mathrm{H}), 6.92-6.84(\mathrm{~m}, 3 \mathrm{H}), 5.60(\mathrm{~s}, 1 \mathrm{H}), 4.17-3.97(\mathrm{~m}, 2 \mathrm{H}), 2.02(\mathrm{~s}, 3 \mathrm{H}), 1.12(\mathrm{t}, J=7.1 \mathrm{~Hz}$, $3 \mathrm{H}) ;{ }^{13} \mathrm{C}$ NMR $\left(101 \mathrm{MHz}, \mathrm{CDCl}_{3}\right) \delta=171.2,148.5,138.4,133.5,131.9,131.3,130.9,129.1$, 128.7, 128.4, 127.9, 127.2, 124.1, 123.2, 121.7, 121.3, 119.7, 66.9, 61.3, 19.1, 13.9 ppm; HRMS (m/z): [M + Na] $]^{+}$calcd for $\mathrm{C}_{23} \mathrm{H}_{21} \mathrm{NO}_{2} \mathrm{Na}^{+} 366.1464$, Found 366.1463.

\section{Ethyl 2,4-dimethyl-5-phenyl-5,6-dihydrophenanthridine-6-carboxylate (5h)}<smiles>CCOC(=O)C1c2ccccc2-c2cc(C)cc(C)c2N1c1ccccc1</smiles>

Pale yellow oil $\left(0.06 \mathrm{~g}, 84 \%\right.$ yield); $\mathrm{R}_{f}=0.6$ (silica, EtOAc:hexanes, 1:9); IR (film) $V_{\max } 2920,1742,1597,1494,1025,764 \mathrm{~cm}^{-1} ;{ }^{1} \mathrm{H}$ NMR $(400 \mathrm{MHz}$, $\left.\mathrm{CDCl}_{3}\right) \delta=7.85(\mathrm{~d}, J=7.8 \mathrm{~Hz}, 1 \mathrm{H}), 7.53(\mathrm{~s}, 1 \mathrm{H}), 7.38$ (ddd, $J=8.2,6.8,2.1$ 
$\mathrm{Hz}, 1 \mathrm{H}), 7.28(\mathrm{td}, J=7.8,3.5 \mathrm{~Hz}, 2 \mathrm{H}), 7.22-7.15(\mathrm{~m}, 2 \mathrm{H}), 6.99(\mathrm{~s}, 1 \mathrm{H}), 6.90-6.86(\mathrm{~m}, 3 \mathrm{H})$, $5.60(\mathrm{~s}, 1 \mathrm{H}), 4.17-3.99(\mathrm{~m}, 2 \mathrm{H}), 2.40(\mathrm{~s}, 3 \mathrm{H}), 2.01(\mathrm{~s}, 3 \mathrm{H}), 1.14(\mathrm{t}, J=7.1 \mathrm{~Hz}, 3 \mathrm{H}) ;{ }^{13} \mathrm{C} \mathrm{NMR}$ $\left(101 \mathrm{MHz}, \mathrm{CDCl}_{3}\right) \delta=171.2,148.6,135.9,133.4,133.3,131.9,131.8,131.4,129.0,128.5$, 128.3, 127.9, 127.0, 123.1, 122.2, 121.0, 119.4, 66.8, 61.2, 21.1, 18.8, 13.9 ppm; HRMS (m/z): $[\mathrm{M}+\mathrm{Na}]^{+}$calcd for $\mathrm{C}_{24} \mathrm{H}_{23} \mathrm{NO}_{2} \mathrm{Na}^{+} 380.1621$, Found 380.1619.

\section{Ethyl 2-fluoro-5-phenyl-5,6-dihydrophenanthridine-6-carboxylate (5i)}

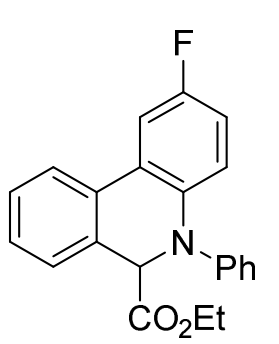

$5 \mathbf{i}$

Yellow oil (0.036 g, $52 \%$ yield); $R_{f}=0.5$ (silica, EtOAc:hexanes, 1:9); IR (film) $v_{\max } 2992,1742,1494,1115,764 \mathrm{~cm}^{-1} ;{ }^{1} \mathrm{H}$ NMR $\left(400 \mathrm{MHz}, \mathrm{CDCl}_{3}\right) \delta$ $=7.74(\mathrm{~d}, J=7.8 \mathrm{~Hz}, 1 \mathrm{H}), 7.49-7.40(\mathrm{~m}, 2 \mathrm{H}), 7.37-7.30(\mathrm{~m}, 4 \mathrm{H}), 7.24-$ $7.18(\mathrm{~m}, 2 \mathrm{H}), 7.15-7.07(\mathrm{~m}, 2 \mathrm{H}), 6.89(\mathrm{td}, J=8.5,2.9 \mathrm{~Hz}, 1 \mathrm{H}), 5.41(\mathrm{~s}$, $1 \mathrm{H}), 4.19-4.02(\mathrm{~m}, 2 \mathrm{H}), 1.14(\mathrm{t}, J=7.1 \mathrm{~Hz}, 3 \mathrm{H}) ;{ }^{13} \mathrm{C}$ NMR $(101 \mathrm{MHz}$, $\left.\mathrm{CDCl}_{3}\right) \delta=171.3,158.7,156.3,146.0,138.1,131.3,130.2,130.1,129.3,128.6,127.8,127.6$, $125.5,125.4,123.1,122.9,121.7,120.4,119.7,119.6,115.5,115.3,110.1,109.9,64.5,61.4$, 14.0 ppm; HRMS (m/z): $[\mathrm{M}+\mathrm{Na}]^{+}$calcd for $\mathrm{C}_{22} \mathrm{H}_{18} \mathrm{FNO}_{2} \mathrm{Na}^{+} 370.1213$, Found 370.1213 .

\section{Ethyl 2-chloro-5-phenyl-5,6-dihydrophenanthridine-6-carboxylate (5j)}

$5 \mathbf{j}$<smiles>CCOC(=O)C1c2ccccc2-c2cc(Cl)ccc2N1c1ccccc1</smiles>

Pale yellow oil ( $0.035 \mathrm{~g}, 48 \%$ yield); $\mathrm{R}_{f}=0.5$ (silica, EtOAc:hexanes, 1:9); IR (film) $V_{\max } 2946,1741,1564,1027,765 \mathrm{~cm}^{-1} ;{ }^{1} \mathrm{H}$ NMR $\left(400 \mathrm{MHz}, \mathrm{CDCl}_{3}\right)$ $\delta=7.76(\mathrm{~d}, J=7.8 \mathrm{~Hz}, 1 \mathrm{H}), 7.72(\mathrm{~d}, J=2.3 \mathrm{~Hz}, 1 \mathrm{H}), 7.42(\mathrm{ddd}, J=8.0$, 6.6, $2.2 \mathrm{~Hz}, 1 \mathrm{H}), 7.34(\mathrm{td}, J=7.8,1.9 \mathrm{~Hz}, 4 \mathrm{H}), 7.21(\mathrm{~d}, J=8.0 \mathrm{~Hz}, 2 \mathrm{H})$, $7.14-7.02(\mathrm{~m}, 3 \mathrm{H}), 5.36(\mathrm{~s}, 1 \mathrm{H}), 4.16-4.03(\mathrm{~m}, 2 \mathrm{H}), 1.13(\mathrm{t}, J=7.1 \mathrm{~Hz}$ $3 \mathrm{H}) ;{ }^{13} \mathrm{C}$ NMR $\left(101 \mathrm{MHz}, \mathrm{CDCl}_{3}\right) \delta=171.2,145.5,140.8,138.1,133.4,130.9,130.2,129.8$, $129.4,128.7,128.4,127.8,127.6,125.5,125.3,123.7,123.5,122.8,122.7,122.4,120.4,119.2$ 118.0, 64.6, 61.5, 14.0 ppm; HRMS (m/z): [M + Na] calcd for $\mathrm{C}_{22} \mathrm{H}_{18} \mathrm{CINO}_{2} \mathrm{Na}^{+}$386.0918, Found 386.0918. 
Ethyl 2,3-dimethoxy-5-phenyl-5,6-dihydrophenanthridine-6-carboxylate (5k)

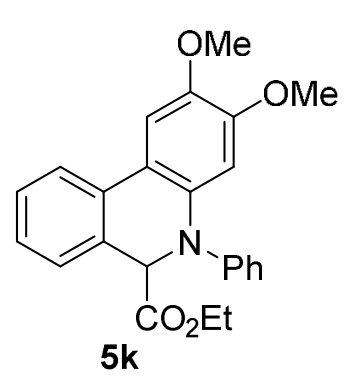

Yellow oil (0.065 g, 84\% yield); $\mathrm{R}_{f}=0.6$ (silica, EtOAc:hexanes, 1:4);

IR (film) $v_{\max } 2936,1738,1564,1099,738 \mathrm{~cm}^{-1} ;{ }^{1} \mathrm{H}$ NMR $(400 \mathrm{MHz}$, $\left.\mathrm{CDCl}_{3}\right) \delta=7.73-7.65(\mathrm{~m}, 1 \mathrm{H}), 7.38(\mathrm{t}, J=7.6 \mathrm{~Hz}, 1 \mathrm{H}), 7.35-7.29$ (m, 3H), $7.26(\mathrm{~s}, 1 \mathrm{H}), 7.25-7.19(\mathrm{~m}, 3 \mathrm{H}), 7.05-7.00(\mathrm{~m}, 1 \mathrm{H}), 6.79(\mathrm{~s}$, 1H), $5.41(\mathrm{~s}, 1 \mathrm{H}), 4.21-3.99(\mathrm{~m}, 2 \mathrm{H}), 3.95(\mathrm{~s}, 3 \mathrm{H}), 3.75(\mathrm{~s}, 3 \mathrm{H}), 1.15$ $(\mathrm{t}, J=7.1 \mathrm{~Hz}, 3 \mathrm{H}) ;{ }^{13} \mathrm{C} \operatorname{NMR}\left(101 \mathrm{MHz}, \mathrm{CDCl}_{3}\right) \delta=171.5,149.8,146.3,144.0,135.6,130.8$, $130.1,129.1,128.4,127.6,126.3,122.3,121.9,121.0,116.9,106.9,102.9,64.6,61.3,56.3$, 55.8, 14.0 ppm; HRMS (m/z): [M + Na] ${ }^{+}$calcd for $\mathrm{C}_{24} \mathrm{H}_{23} \mathrm{NO}_{4} \mathrm{Na}^{+}$412.1519, Found 412.1517.

Ethyl 2,3-dimethyl-5-phenyl-5,6-dihydrophenanthridine-6-carboxylate (5I)

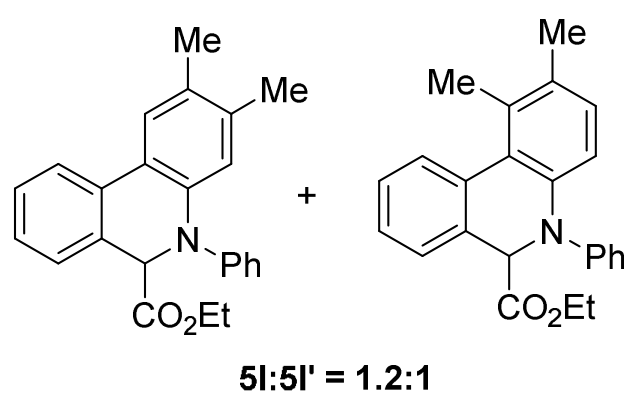

Pale yellow oil $(0.056 \mathrm{~g}, 78 \%$ yield (inseparable mixture with a ratio of $1.2: 1$, determined by ${ }^{1} \mathrm{H}$ NMR)); $\mathrm{R}_{f}=0.4$ (silica, EtOAc:hexanes, 1:9); IR (film) $\mathrm{v}_{\max }$ 2928, 1742, 1495, 1114, $739 \mathrm{~cm}^{-1} ;{ }^{1} \mathrm{H}$ NMR $(400$ $\left.\mathrm{MHz}, \mathrm{CDCl}_{3}\right) \delta=7.85-7.77(\mathrm{~m}), 7.57(\mathrm{~d}), 7.45-$

$7.21(\mathrm{~m}), 7.13-6.98(\mathrm{~m}), 5.40(\mathrm{~m}), 4.16(\mathrm{~m}), 4.06(\mathrm{~m}), 2.59(\mathrm{~s}), 2.33(\mathrm{~s}), 2.31(\mathrm{~s}), 2.21(\mathrm{~s}), 1.18$ (t), $1.13(\mathrm{t}) ;{ }^{13} \mathrm{C}$ NMR $\left(101 \mathrm{MHz}, \mathrm{CDCl}_{3}\right) \delta=171.5,171.2,146.2,146.1,141.0,139.6,137.3$, $134.1,133.5,131.2,131.0,130.6,130.3,129.5,129.1,129.0,128.8,128.3,127.7,127.5,127.2$, $127.0,126.5,126.2,125.6,124.6,122.5,122.3,122.2,121.8,121.7,121.4,119.2,115.9,64.7$ 64.6, 61.2, 61.1, 20.4, 19.9, 19.4, 19.2, 14.0, 13.9 ppm; HRMS (m/z): $[\mathrm{M}+\mathrm{Na}]^{+}$calcd for $\mathrm{C}_{24} \mathrm{H}_{23} \mathrm{NO}_{2} \mathrm{Na}^{+} 380.1621$, Found 380.1619.

Ethyl 5-(benzo[d][1,3]dioxol-5-yl)-2-methoxy-5,6-dihydro-[1,3]dioxolo[4,5j]phenanthridine-6-carboxylate $(5 \mathrm{~m})$ 


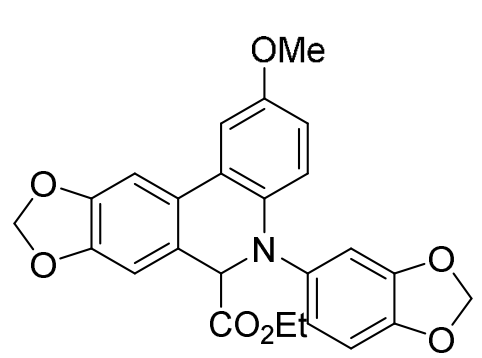

$5 \mathrm{~m}$

Pale yellow oil $\left(0.064 \mathrm{~g}, 72 \%\right.$ yield); $\mathrm{R}_{f}=0.6$ (silica, EtOAc:hexanes, 1:4); IR (film) $v_{\max }$ 2998, 1742, 1592, 1266, 1001, $749 \mathrm{~cm}^{-1} ;{ }^{1} \mathrm{H}$ NMR $\left(400 \mathrm{MHz}, \mathrm{CDCl}_{3}\right) \delta=7.21(\mathrm{~s}, 1 \mathrm{H})$, $7.13(\mathrm{~d}, J=2.8 \mathrm{~Hz}, 1 \mathrm{H}), 6.93(\mathrm{~d}, J=8.9 \mathrm{~Hz}, 1 \mathrm{H}), 6.79(\mathrm{~s}, 1 \mathrm{H})$, $6.77-6.74(\mathrm{~m}, 2 \mathrm{H}), 6.71(\mathrm{dd}, J=8.9,2.8 \mathrm{~Hz}, 1 \mathrm{H}), 6.62(\mathrm{dd}, J$ = 8.4, 2.2 Hz, 1H), $5.99(\mathrm{~s}, 2 \mathrm{H}), 5.94(\mathrm{~d}, \mathrm{~J}=12.1 \mathrm{~Hz}, 2 \mathrm{H}), 5.15(\mathrm{~s}, 1 \mathrm{H}), 4.16-3.98(\mathrm{~m}, 2 \mathrm{H})$, $3.82(\mathrm{~s}, 3 \mathrm{H}), 1.14(\mathrm{t}, J=7.1 \mathrm{~Hz}, 3 \mathrm{H}) ;{ }^{13} \mathrm{C} \operatorname{NMR}\left(101 \mathrm{MHz}, \mathrm{CDCl}_{3}\right) \delta=171.5,153.7,148.2$, 148.0, 147.1, 143.6, 141.3, 135.7, 125.3, 125.1, 124.7, 119.1, 115.3, 114.1, 108.3, 107.7, 104.8, 103.1, 101.2, 101.1, 65.3, 61.2, 55.6, 14.0 ppm; HRMS (m/z): $[\mathrm{M}+\mathrm{Na}]^{+}$calcd for $\mathrm{C}_{25} \mathrm{H}_{21} \mathrm{NO}_{7} \mathrm{Na}^{+}$ 470.1210, Found 470.1206.

Ethyl 5-(3,4-dimethoxyphenyl)-2,8,9-trimethoxy-5,6-dihydrophenanthridine-6-carboxylate $(5 n)$<smiles>CCOC(=O)c1ccc(OC)c(OC)c1</smiles>
Pale yellow oil $\left(0.07 \mathrm{~g}, 74 \%\right.$ yield); $R_{f}=0.5$ (silica, EtOAc:hexanes, 1:4); IR (film) $\mathrm{V}_{\max } 2994,1745,1508,1346$, 1128, $765 \mathrm{~cm}^{-1} ;{ }^{1} \mathrm{H}$ NMR $\left(400 \mathrm{MHz}, \mathrm{CDCl}_{3}\right) \delta=7.23(\mathrm{~s}, 1 \mathrm{H})$, $7.21(\mathrm{~d}, J=2.9 \mathrm{~Hz}, 1 \mathrm{H}), 6.92(\mathrm{~d}, J=8.8 \mathrm{~Hz}, 1 \mathrm{H}), 6.86-6.80$ $(\mathrm{m}, 3 \mathrm{H}), 6.71$ (ddd, $J=8.9,4.9,2.7 \mathrm{~Hz}, 2 \mathrm{H}), 5.23(\mathrm{~s}, 1 \mathrm{H})$, 4.08 (q, J = 7.1 Hz, 2H), 3.97 (s, 3H), 3.90 (s, 3H), 3.87 (s, 3H), $3.84(\mathrm{~s}, 3 \mathrm{H}), 3.79(\mathrm{~s}, 3 \mathrm{H}), 1.13$ $(\mathrm{t}, J=7.1 \mathrm{~Hz}, 3 \mathrm{H}) ;{ }^{13} \mathrm{C} \operatorname{NMR}\left(101 \mathrm{MHz}, \mathrm{CDCl}_{3}\right) \delta=171.8,153.4,149.3,148.8,145.4,140.2$, 136.0, 124.5, 123.8, 123.7, 118.5, 114.4, 113.3, 111.7, 110.3, 108.8, 107.6, 105.7, 64.9, 61.1, 56.1, 56.0, 55.8, 55.7, 14.1 ppm; HRMS (m/z): $[\mathrm{M}+\mathrm{Na}]^{+}$calcd for $\mathrm{C}_{27} \mathrm{H}_{29} \mathrm{NO}_{7} \mathrm{Na}^{+}$502.1836, Found 502.1847.

Ethyl 5-(2,3-dihydro-1H-inden-5-yl)-2-methoxy-6,8,9,10-tetrahydro-5Hcyclopenta[j]phenanthridine-6-carboxylate (5o) 


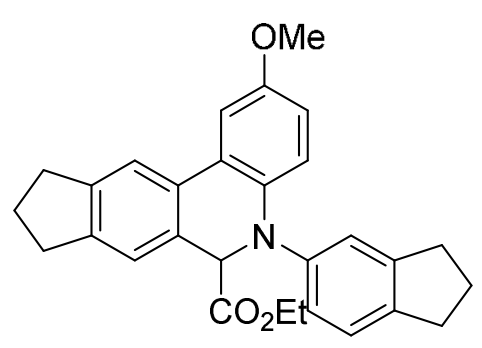

50

Yellow oil $\left(0.074 \mathrm{~g}, \quad 84 \%\right.$ yield); $\mathrm{R}_{f}=0.3 \quad$ (silica, EtOAc:hexanes, 1:9); IR (film) $v_{\max } 3012,1745$, 1592, 1496, 1028, $728 \mathrm{~cm}^{-1} ;{ }^{1} \mathrm{H}$ NMR $\left(400 \mathrm{MHz}, \mathrm{CDCl}_{3}\right) \delta=7.66(\mathrm{~s}, 1 \mathrm{H})$, $7.31(\mathrm{~d}, J=2.8 \mathrm{~Hz}, 1 \mathrm{H}), 7.20(\mathrm{~s}, 1 \mathrm{H}), 7.16(\mathrm{~d}, J=8.1 \mathrm{~Hz}, 1 \mathrm{H})$, $7.12-7.04(\mathrm{~m}, 2 \mathrm{H}), 6.99(\mathrm{dd}, J=8.1,2.2 \mathrm{~Hz}, 1 \mathrm{H}), 6.76(\mathrm{dd}, J$

$=8.9,2.8 \mathrm{~Hz}, 1 \mathrm{H}), 5.34(\mathrm{~s}, 1 \mathrm{H}), 4.19-4.02(\mathrm{~m}, 2 \mathrm{H}), 3.87(\mathrm{~s}, 3 \mathrm{H}), 3.07-2.92(\mathrm{~m}, 4 \mathrm{H}), 2.89(\mathrm{t}, J$ $=7.4 \mathrm{~Hz}, 4 \mathrm{H}), 2.16-2.06(\mathrm{~m}, 4 \mathrm{H}), 1.15(\mathrm{t}, J=7.1 \mathrm{~Hz}, 3 \mathrm{H}) ;{ }^{13} \mathrm{C} \mathrm{NMR}\left(101 \mathrm{MHz}, \mathrm{CDCl}_{3}\right) \delta=$ 172.0, 153.6, 145.2, 145.2, 144.6, 143.7, 138.2, 135.9, 129.7, 129.0, 125.6, 124.5, 123.3, 119.7, 118.6, 117.6, 114.2, 108.2, 65.2, 61.0, 55.5, 33.0, 32.7, 32.6, 32.1, 25.6, 25.4, 14.0 ppm; HRMS (m/z): $[\mathrm{M}+\mathrm{Na}]^{+}$calcd for $\mathrm{C}_{29} \mathrm{H}_{29} \mathrm{NO}_{3} \mathrm{Na}^{+}$462.2039, Found 462.2036.

Ethyl 2-methoxy-5-(naphthalen-2-yl)-5,6-dihydrobenzo[j]phenanthridine-6-carboxylate $(5 p)$

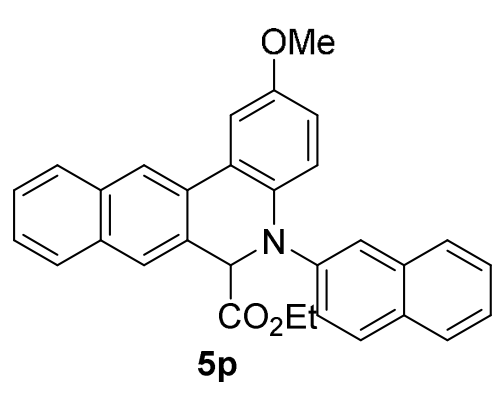

Pale yellow oil $\left(0.07 \mathrm{~g}, \quad 76 \%\right.$ yield); $\mathrm{R}_{f}=0.3$ (silica, EtOAc:hexanes, 1:9); IR (film) $v_{\max } 2982,1748,1498,1285$, 1062, $810 \mathrm{~cm}^{-1} ;{ }^{1} \mathrm{H}$ NMR $\left(400 \mathrm{MHz}, \mathrm{CDCl}_{3}\right) \delta=8.26(\mathrm{~s}, 1 \mathrm{H})$, $7.95-7.90(m, 1 H), 7.84-7.82(m, 2 H), 7.79-7.70(m, 3 H)$, $7.53(\mathrm{~d}, J=3.0 \mathrm{~Hz}, 1 \mathrm{H}), 7.51-7.42(\mathrm{~m}, 5 \mathrm{H}), 7.38-7.34(\mathrm{~m}$, 1H), $7.21(\mathrm{~d}, J=8.9 \mathrm{~Hz}, 1 \mathrm{H}), 6.84(\mathrm{dd}, J=8.9,2.9 \mathrm{~Hz}, 1 \mathrm{H}), 5.73(\mathrm{~s}, 1 \mathrm{H}), 4.22-4.04(\mathrm{~m}, 2 \mathrm{H})$, $3.92(\mathrm{~s}, 3 \mathrm{H}), 1.14(\mathrm{t}, J=7.1 \mathrm{~Hz}, 3 \mathrm{H}) ;{ }^{13} \mathrm{C} \operatorname{NMR}\left(101 \mathrm{MHz}, \mathrm{CDCl}_{3}\right) \delta=171.4,154.6,144.1$, $135.6,134.5,133.5,132.6,130.6,129.7,128.9,128.6,128.0,127.6,127.5,126.8,126.4,126.1$, $125.9,124.2,122.2,121.7,120.5,116.0,115.2,109.0,65.0,61.5,55.7,14.0$ ppm; HRMS (m/z): $[\mathrm{M}+\mathrm{Na}]^{+}$calcd for $\mathrm{C}_{31} \mathrm{H}_{25} \mathrm{NO}_{3} \mathrm{Na}^{+} 482.1726$, Found 482.1727.

Ethyl 5-(2,5-dimethylphenyl)-2-methoxy-7,10-dimethyl-5,6-dihydrophenanthridine-6carboxylate $(5 q)$ 


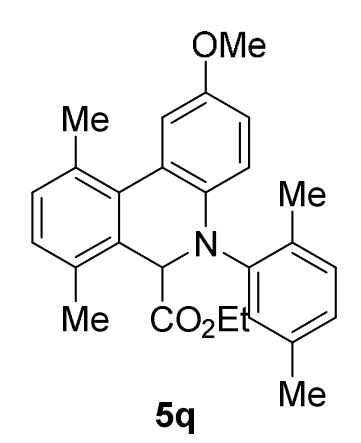

Yellow oil $\left(0.068 \mathrm{~g}, 82 \%\right.$ yield); $\mathrm{R}_{f}=0.4$ (silica, EtOAc:hexanes, 1:9); IR (film) $\mathrm{V}_{\max } 2925,1741,1502,1471,1039,865 \mathrm{~cm}^{-1} ;{ }^{1} \mathrm{H}$ NMR $(400 \mathrm{MHz}$, $\left.\mathrm{CDCl}_{3}\right) \delta=7.32(\mathrm{~d}, J=2.9 \mathrm{~Hz}, 1 \mathrm{H}), 7.19-7.12(\mathrm{~m}, 2 \mathrm{H}), 7.07(\mathrm{~d}, J=7.6$ $\mathrm{Hz}, 2 \mathrm{H}), 6.93(\mathrm{~d}, J=7.6 \mathrm{~Hz}, 1 \mathrm{H}), 6.73(\mathrm{dd}, J=8.9,2.8 \mathrm{~Hz}, 1 \mathrm{H}), 6.52(\mathrm{~d}, J$ $=8.8 \mathrm{~Hz}, 1 \mathrm{H}), 5.37(\mathrm{~s}, 1 \mathrm{H}), 4.19-3.94(\mathrm{~m}, 2 \mathrm{H}), 3.81(\mathrm{~s}, 3 \mathrm{H}), 2.68(\mathrm{~s}$, $3 \mathrm{H}), 2.45(\mathrm{~s}, 3 \mathrm{H}), 2.37(\mathrm{~s}, 3 \mathrm{H}), 1.87(\mathrm{~s}, 3 \mathrm{H}), 1.09(\mathrm{t}, J=7.1 \mathrm{~Hz}, 3 \mathrm{H}) ;{ }^{13} \mathrm{C}$ NMR $\left(101 \mathrm{MHz}, \mathrm{CDCl}_{3}\right) \delta=172.0,152.1,144.1,138.6,136.6,135.1,131.8,131.6,131.4$ 131.0, 130.5, 128.8, 125.8, 125.2, 124.7, 117.9, 114.2, 113.5, 62.5, 60.9, 55.6, 23.4, 21.2, 19.6, 18.6, 13.9 ppm; HRMS (m/z): [M + Na] calcd for $\mathrm{C}_{27} \mathrm{H}_{29} \mathrm{NO}_{3} \mathrm{Na}^{+}$438.2039, Found 438.2038.

Ethyl 2,10-dimethoxy-5-(3-methoxyphenyl)-5,6-dihydrophenanthridine-6-carboxylate (5r) and Ethyl 2,7-dimethoxy-5-(3-methoxyphenyl)-5,6-dihydrophenanthridine-6-carboxylate $\left(5 r^{\prime}\right)$<smiles>CCOC(=O)C1c2c(OC)cccc2-c2cc(OC)ccc2N1c1cccc(OC)c1N1c2cccc(OC)c2-c2c(OC)cccc2C1C(=O)OC</smiles>

Pale yellow oil $(0.058 \mathrm{~g}, 69 \%$ yield (inseparable mixture of isomers with a ratio of $3: 1$, determined by ${ }^{1} \mathrm{H}$ NMR)) ${ }^{4} ; \mathrm{R}_{f}=0.4$ (silica, EtOAc:hexanes, 1:4); IR (film) $v_{\max } 3056,1737,1507,1493,1226$, $750 \mathrm{~cm}^{-1} ;{ }^{1} \mathrm{H} \mathrm{NMR}\left(400 \mathrm{MHz}, \mathrm{CDCl}_{3}\right) \delta=8.03(\mathrm{~d}, J$ $=3.0 \mathrm{~Hz}), 7.38-7.33(\mathrm{~m}), 7.30(\mathrm{~d}, J=3.0 \mathrm{~Hz})$,

$7.27-7.22(\mathrm{~m}), 7.17(\mathrm{t}, J=8.1 \mathrm{~Hz}), 6.97-6.95(\mathrm{~m}), 6.87-6.82(\mathrm{~m}), 6.80-6.72(\mathrm{~m}), 6.54-$ $6.50(\mathrm{~m}), 5.95(\mathrm{~s}), 5.39(\mathrm{~s}), 4.13-3.99(\mathrm{~m}), 3.95(\mathrm{~s}), 3.86(\mathrm{~s}), 3.85(\mathrm{~s}), 3.83(\mathrm{~s}), 3.76(\mathrm{~s}), 3.75$ (s), $1.11-1.07(\mathrm{~m}) ;{ }^{13} \mathrm{C}$ NMR $\left(101 \mathrm{MHz}, \mathrm{CDCl}_{3}\right) \delta=171.4,171.3,160.3,156.5,154.4,153.6$, 148.5, 147.7, 135.2, 135.1, 129.7, 129.6, 128.8, 127.9, 126.6, 124.9, 121.5, 120.5, 120.4, 119.8, 115.3, 114.9, 114.0, 113.9, 112.9, 112.5, 111.2, 109.4, 108.9, 107.0, 106.9, 106.5, 106.1, 64.4, $61.3,61.1,58.0,55.7,55.67,55.62,55.5,55.2,14.8,14.0 \mathrm{ppm} ; \mathrm{HRMS}(\mathrm{m} / \mathrm{z}):[\mathrm{M}+\mathrm{Na}]^{+}$calcd for $\mathrm{C}_{25} \mathrm{H}_{25} \mathrm{NO}_{5} \mathrm{Na}^{+} 442.1624$, Found 442.1627 . 
Ethyl 2-methoxy-8-methyl-5-(p-tolyl)-5,6-dihydrophenanthridine-6-carboxylate and three other possible isomers (5s)

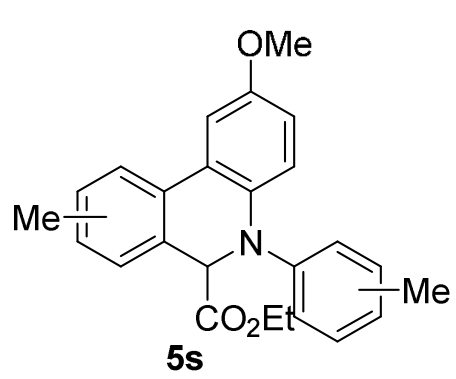

Mixture of 4 Isomers

$(1: 1: 1: 1)$
Pale yellow oil $(0.068 \mathrm{~g}, 82 \%$ yield (inseparable mixture with a ratio of $1: 1: 1: 1$, determined by ${ }^{1} \mathrm{H}$ NMR)); $\mathrm{R}_{f}=0.4$ (silica, EtOAc:hexanes, 1:9); IR (film) $v_{\max } 2996,1742,1596,1495,761$ $\mathrm{cm}^{-1} ;{ }^{1} \mathrm{H} \mathrm{NMR}\left(400 \mathrm{MHz}, \mathrm{CDCl}_{3}\right) \delta=7.68(\mathrm{~d}, \mathrm{~J}=8.0 \mathrm{~Hz}), 7.60$ (s), $7.31(\mathrm{dd}, J=7.3,3.0 \mathrm{~Hz}), 7.28-7.19(\mathrm{~m}), 7.19-7.13(\mathrm{~m}), 7.13-$ $7.04(\mathrm{~m}), 7.01(\mathrm{~s}), 6.99(\mathrm{~s}), 6.84(\mathrm{~d}, J=7.5 \mathrm{~Hz}), 6.81-6.73(\mathrm{~m})$,

$5.37(\mathrm{~d}, J=4.5 \mathrm{~Hz}), 5.32(\mathrm{~d}, J=5.1 \mathrm{~Hz}), 4.17-4.00(\mathrm{~m}), 3.87$ (s), $3.86(\mathrm{~s}, 1 \mathrm{H}), 3.85(\mathrm{~s}), 2.44$ (s), 2.39 (s), 2.33 (s), $1.16-1.12(\mathrm{~m}) ;{ }^{13} \mathrm{C}$ NMR (101 MHz, $\left.\mathrm{CDCl}_{3}\right) \delta=171.7,171.6,154.1$, 154.0, 153.8, 153.7, 146.7, 146.6, 144.1, 144.0, 138.9, 138.1, 138.0, 137.3, 137.2, 136.0, 135.7, $135.5,135.2,132.0,131.9,131.5,131.4,130.62,130.60,130.59,129.7,129.3,128.9,128.8$, $128.7,128.2,128.16,128.1,127.5,127.4,125.7,125.5,125.2,125.1,123.4,123.0,122.9$, $122.8,122.7,121.5,121.5,121.46,121.41,120.2,119.5,118.0,117.9,114.7,114.3,108.5$, 108.4, 64.7, 64.5, 64.4, 64.3, 61.2, 55.6, 21.5, 21.4, 21.1, 20.7, 14.0 ppm; HRMS (m/z): [M + $\mathrm{Na}]^{+}$calcd for $\mathrm{C}_{25} \mathrm{H}_{25} \mathrm{NO}_{3} \mathrm{Na}^{+}$410.1726, Found 410.1725.

\section{Procedure for ADAR with isolated imine 6a}

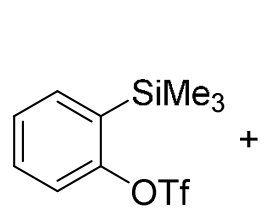

$3 a$

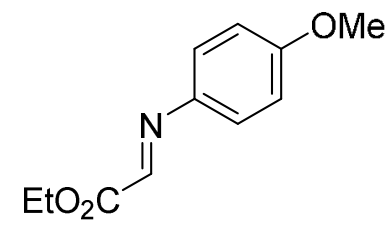

$6 a$
KF, 18-crown-6

$\mathrm{CH}_{3} \mathrm{CN}, 25^{\circ} \mathrm{C}$<smiles>CCOC(=O)C1c2ccccc2-c2cc(OC)ccc2N1c1ccccc1</smiles>

$5 a$

To a flame-dried screw-capped test tube equipped with a magnetic stir bar was charged with the imine $6 \mathbf{a}(0.25 \mathrm{mmol}), \mathrm{KF}(1.2 \mathrm{mmol})$, and 18-crown-6 $(1.2 \mathrm{mmol})$ and subjected to vacuum for 
$30 \mathrm{~min}$. The mixture was then placed under an argon atmosphere, and anhydrous $\mathrm{MeCN}(2.0$ $\mathrm{mL}$ ) was added. To this stirred solution kept at room temperature was added the aryne precursor $3 \mathrm{a}(0.75 \mathrm{mmol})$ in one portion, and the resulting reaction mixture was stirred at rt for 2 h. Then, the reaction mixture was diluted with EtOAc and hydrolyzed with water. The organic layer was separated, and the aqueous layer was extracted twice with EtOAc. The combined organic layers were washed with brine, dried over anhydrous sodium sulfate, filtered, and concentrated under vacuum to give the crude product. Flash chromatography of this material $\mathbf{a} \square$ orded pure product $\mathbf{5 a}$ in $89 \%$ yield.

\section{General procedure for the optimization of the reaction conditions for oxoimidazolidine derivatives 7 .}

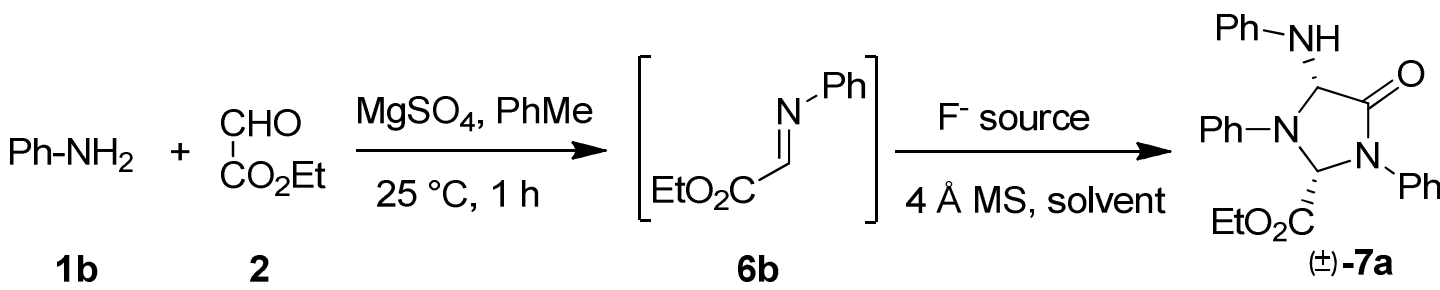

To a flame-dried screw-capped test tube equipped with a magnetic stir bar containing the aniline (1b, $0.2 \mathrm{mmol})$ in toluene $(2.0 \mathrm{~mL})$ was added $\mathrm{MgSO}_{4}$ (5 equiv) under argon atmosphere. The mixture was stirred, and then ethyl glyoxalate (2, 50\% in toluene) was added slowly. The resultant reaction mixture was kept in stirring for $1 \mathrm{~h}, \mathrm{MgSO}_{4}$ was removed by filtration, and toluene was distilled under reduced pressure to yield the crude compound. The crude compound was dissolved in $2.0 \mathrm{~mL}$ of solvent under argon atmosphere, $4 \AA \mathrm{MS}(300 \mathrm{mg} / \mathrm{mmol}$ of $1 \mathrm{~b})$ and fluoride source or base was added. Then the reaction mixture was stirred at $25^{\circ} \mathrm{C}$ till the completion of reaction (monitored by TLC). After the reaction solution was filtered and concentrated under reduced pressure, the residue was purified by flash chromatography on silica gel (eluent: EtOAc/petroleum ether $=1 / 10$ ) to afford the corresponding pure product. 


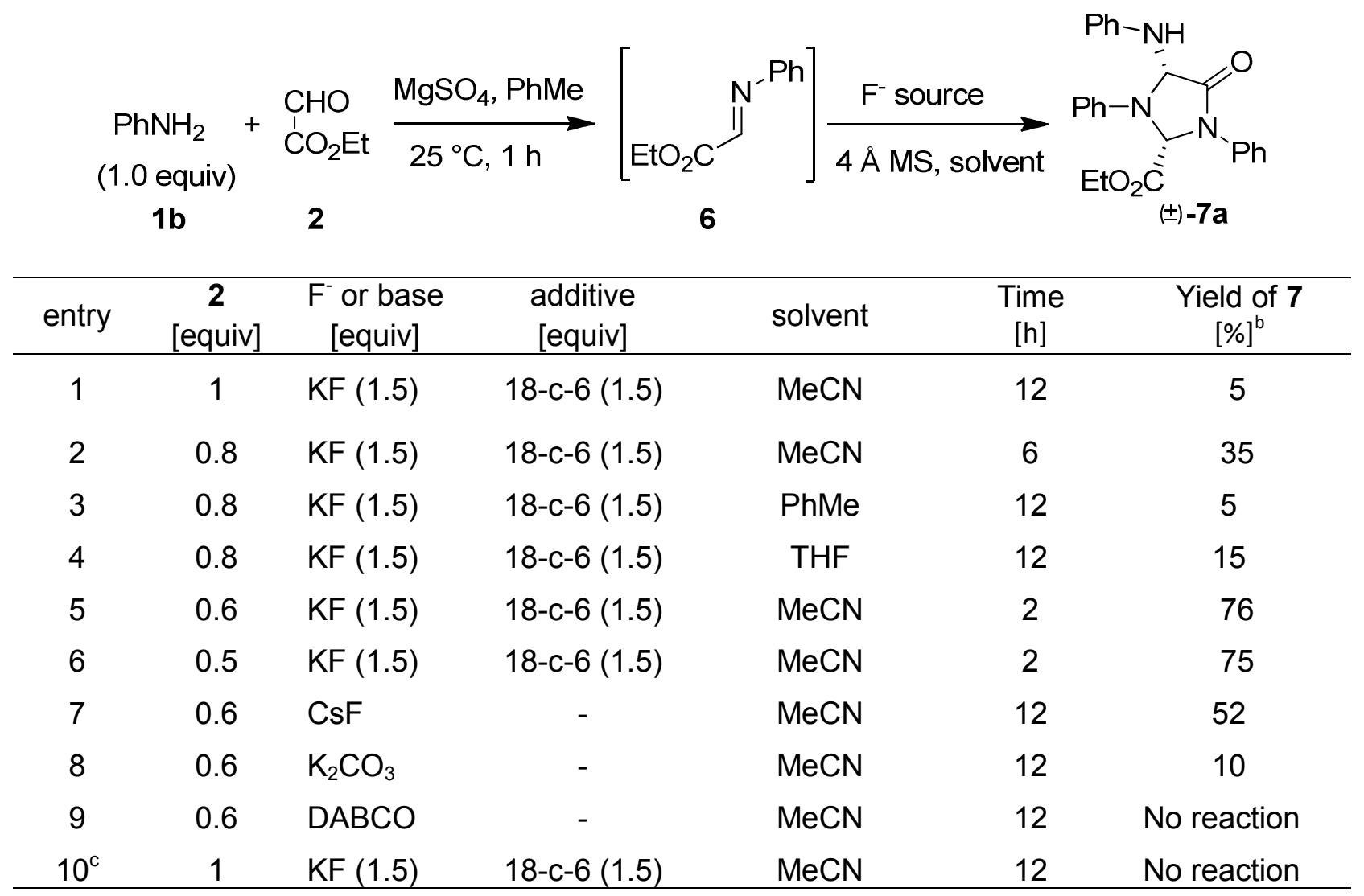

${ }^{a} \mathbf{1 b}(0.20 \mathrm{mmol}), \mathbf{2}, \mathrm{MgSO}_{4}$ (5.0 equiv), $\mathrm{PhMe}(2.0 \mathrm{~mL}), 1 \mathrm{~h}$ then filtered and filtrate was evaporated, followed by $4 \AA \mathrm{MS}(300 \mathrm{mg} / \mathrm{mmol}$ of $1 \mathrm{~b}), \mathrm{KF}, 18$-crown-6, MeCN $(2.0 \mathrm{~mL}), 25^{\circ} \mathrm{C}, 2 \mathrm{~h}$. ${ }^{\mathrm{b}}$ Yields of the isolated products with respect to $\mathbf{1} \mathbf{b}$. ' After formation of imine 0.5 equiv of $\mathbf{1} \mathbf{b}$ was added additionally.

\section{General procedure for the synthesis of oxoimidazolidine derivatives 7.}

To a flame-dried screw-capped test tube equipped with a magnetic stir bar containing the anilines $(1,0.2 \mathrm{mmol})$ in toluene $(2.0 \mathrm{~mL})$ was added $\mathrm{MgSO}_{4}(5$ equiv) under argon atmosphere. The mixture was stirred, and then ethyl glyoxalate $(2,50 \%$ in toluene, $0.12 \mathrm{mmol})$ was added slowly. The resultant reaction mixture was kept in stirring for $1 \mathrm{~h}, \mathrm{MgSO}_{4}$ was removed by filtration, and toluene was distilled under reduced pressure to yield the crude compound. The crude compound was dissolved in $2.0 \mathrm{~mL}$ of solvent under argon atmosphere, $4 \AA$ MS (300 $\mathrm{mg} / \mathrm{mmol}$ of 1$)$, $18-$ crown-6 $(0.3 \mathrm{mmol})$ and $\mathrm{KF}(0.3 \mathrm{mmol})$ was added. Then the reaction mixture was stirred at $25^{\circ} \mathrm{C}$ till the completion of reaction (monitored by TLC; typically after $2 \mathrm{~h}$ ). 
After the reaction solution was filtered and concentrated under reduced pressure, the residue was purified by flash chromatography on silica gel (eluent: EtOAc/petroleum ether $=1 / 10$ ) to afford the corresponding pure products $7 \mathbf{a - k}$ in moderate to good yields.

\section{Ethyl 4-oxo-1,3-diphenyl-5-(phenylamino)imidazolidine-2-carboxylate (7a) ${ }^{3}$}

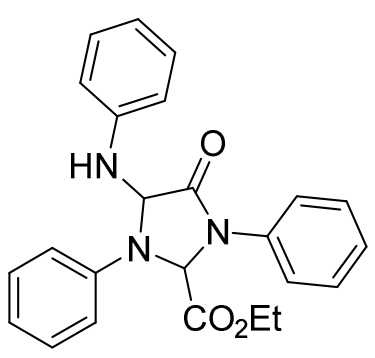

$7 a$
White solid $\left(0.061 \mathrm{~g}, 76 \%\right.$ yield, m.p. $69-71{ }^{\circ} \mathrm{C}$ ); $\mathrm{R}_{f}=0.5$ (silica, EtOAc:hexanes, 1.5:8.5); IR (film) $\mathrm{v}_{\max } 3413,2920,1732,1599,1498$, 1198, $752 \mathrm{~cm}^{-1} ;{ }^{1} \mathrm{H}$ NMR $\left(400 \mathrm{MHz}, \mathrm{CDCl}_{3}\right) \delta=7.59(\mathrm{~d}, J=8.5 \mathrm{~Hz}$, 2H), $7.42(\mathrm{t}, J=7.8 \mathrm{~Hz}, 2 \mathrm{H}), 7.31-7.23(\mathrm{~m}, 5 \mathrm{H}), 6.95(\mathrm{~d}, J=8.5 \mathrm{~Hz}$, 2H), $6.90(\mathrm{t}, J=7.5 \mathrm{~Hz}, 1 \mathrm{H}), 6.85(\mathrm{t}, J=7.4 \mathrm{~Hz}, 1 \mathrm{H}), 6.73(\mathrm{~d}, J=8.1$

$\mathrm{Hz}, 2 \mathrm{H}), 5.93(\mathrm{~s}, 1 \mathrm{H}), 5.67(\mathrm{~d}, J=8.5 \mathrm{~Hz}, 1 \mathrm{H}), 4.56(\mathrm{~d}, J=8.6 \mathrm{~Hz}, 1 \mathrm{H}), 4.18(\mathrm{q}, J=7.1 \mathrm{~Hz}, 2 \mathrm{H})$, $1.12(\mathrm{t}, J=7.1 \mathrm{~Hz}, 3 \mathrm{H}) ;{ }^{13} \mathrm{C} \operatorname{NMR}\left(101 \mathrm{MHz}, \mathrm{CDCl}_{3}\right) \delta=169.5,168.1,145.4,141.5,134.7$, $129.7,129.4,129.3,126.9,122.5,120.1,119.2,114.3,112.5,73.6,68.5,62.7,13.9$ ppm; HRMS (m/z): $[\mathrm{M}+\mathrm{Na}]^{+}$calcd for $\mathrm{C}_{24} \mathrm{H}_{23} \mathrm{~N}_{3} \mathrm{O}_{3} \mathrm{Na}^{+}$424.1631, Found 424.1629.

\section{Ethyl 1,3-bis(4-fluorophenyl)-4-((4-fluorophenyl)amino)-5-oxoimidazolidine-2-carboxylate} $(7 b)^{3}$

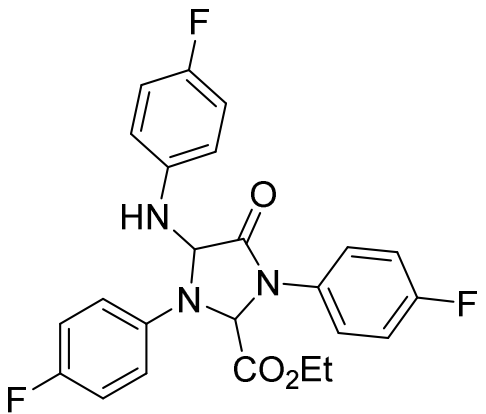

$\mathbf{7 b}$
Pale yellow solid $\left(0.071 \mathrm{~g}, 78 \%\right.$ yield, m.p. $\left.82-84{ }^{\circ} \mathrm{C}\right) ; \mathrm{R}_{f}=0.5$ (silica, EtOAc:hexanes, 1.5:8.5); IR (film) $\mathrm{v}_{\max }$ 3413, 1732, 1614, $1511,1401,1233,1164,820 \mathrm{~cm}^{-1} ;{ }^{1} \mathrm{H}$ NMR $\left(400 \mathrm{MHz}, \mathrm{CDCl}_{3}\right) \delta=$ $7.55-7.48(\mathrm{~m}, 2 \mathrm{H}), 7.12(\mathrm{t}, J=8.7 \mathrm{~Hz}, 2 \mathrm{H}), 7.01-6.94(\mathrm{~m}, 4 \mathrm{H})$, $6.92-6.86(\mathrm{~m}, 2 \mathrm{H}), 6.71-6.61(\mathrm{~m}, 2 \mathrm{H}), 5.81(\mathrm{~s}, 1 \mathrm{H}), 5.49(\mathrm{~d}, J=$ $8.4 \mathrm{~Hz}, 1 \mathrm{H}), 4.47(\mathrm{~d}, J=8.6 \mathrm{~Hz}, 1 \mathrm{H}), 4.24-4.14(\mathrm{~m}, 2 \mathrm{H}), 1.14$ (td, $J=7.1,1.2 \mathrm{~Hz}, 3 \mathrm{H}) ;{ }^{13} \mathrm{C}$ NMR $\left(101 \mathrm{MHz}, \mathrm{CDCl}_{3}\right) \delta=169.3,168.1,162.3,159.8,158.4,158.1$, $156.0,155.8,141.4,141.4,137.9,137.8,130.5,130.5,124.9,124.8,116.5,116.4,116.3,116.2$ 
$116.1,116.0,115.9,115.8,113.7,113.6,74.0,69.6,62.9,13.9$ ppm; HRMS (m/z): [M + Na] ${ }^{+}$ calcd for $\mathrm{C}_{24} \mathrm{H}_{20} \mathrm{~N}_{3} \mathrm{O}_{3} \mathrm{~F}_{3} \mathrm{Na}^{+}$478.1349, Found 478.1348.

Ethyl 1,3-bis(4-bromophenyl)-4-((4-bromophenyl)amino)-5-oxoimidazolidine-2carboxylate $(7 \mathrm{c})^{3}$

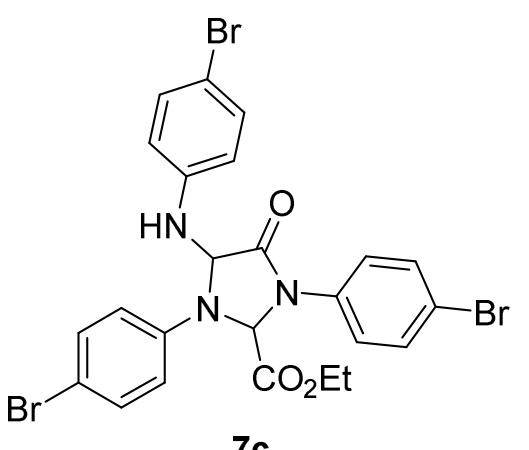

White solid (0.096 g, $76 \%$ yield, m.p. $\left.102-104{ }^{\circ} \mathrm{C}\right) ; \mathrm{R}_{f}=0.5$ (silica, EtOAc:hexanes, 1.5:8.5); IR (film) $v_{\max } 3415,1731,1619$, 1493, 1286, 1010, $809 \mathrm{~cm}^{-1} ;{ }^{1} \mathrm{H}$ NMR $\left(400 \mathrm{MHz} \mathrm{CDCl}_{3}\right) \delta=7.56$ $(\mathrm{d}, J=8.5 \mathrm{~Hz}, 2 \mathrm{H}), 7.49(\mathrm{~d}, J=8.6 \mathrm{~Hz}, 2 \mathrm{H}), 7.36(\mathrm{t}, J=8.3 \mathrm{~Hz}$, $4 \mathrm{H}), 6.82(\mathrm{~d}, J=8.3 \mathrm{~Hz}, 2 \mathrm{H}), 6.57(\mathrm{~d}, J=8.4 \mathrm{~Hz}, 2 \mathrm{H}), 5.87(\mathrm{~s}$, 1H), $5.56(\mathrm{~d}, J=8.8 \mathrm{~Hz}, 1 \mathrm{H}), 4.60(\mathrm{~d}, J=8.8 \mathrm{~Hz}, 1 \mathrm{H}), 4.22(\mathrm{q}, J$ $=7.1 \mathrm{~Hz}, 2 \mathrm{H}), 1.17(\mathrm{t}, J=7.1 \mathrm{~Hz}, 3 \mathrm{H}) ;{ }^{13} \mathrm{C} \operatorname{NMR}\left(101 \mathrm{MHz}, \mathrm{CDCl}_{3}\right) \delta=169.2,167.5,144.1$, $140.1,133.6,132.6,132.5,132.3,123.6,120.4,115.9,114.2,112.8,111.4,73.1,68.2,63.2$, $14.0 \mathrm{ppm}$.

Ethyl 1,3-bis(4-chlorophenyl)-4-((4-chlorophenyl)amino)-5-oxoimidazolidine-2-carboxylate $(7 d)^{3}$

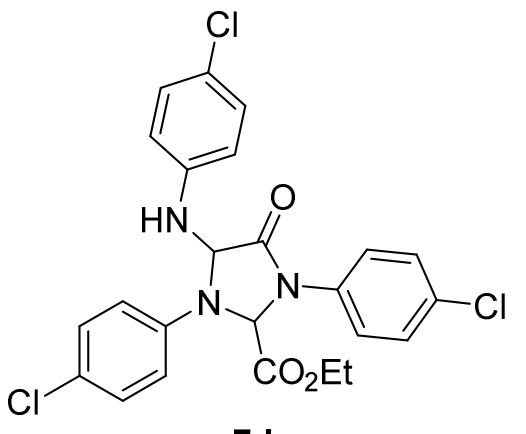

7d

Colorless oil $\left(0.079 \mathrm{~g}, \quad 78 \%\right.$ yield); $R_{f}=0.5$ (silica, EtOAc:hexanes, 1.5:8.5); IR (film) $v_{\max } 3400,1733,1600,1496$, 1287, 1201,1095, 1014, $814 \mathrm{~cm}^{-1} ;{ }^{1} \mathrm{H}$ NMR $\left(400 \mathrm{MHz}, \mathrm{CDCl}_{3}\right) \delta$ $=7.54(\mathrm{~d}, J=8.7 \mathrm{~Hz}, 2 \mathrm{H}), 7.40(\mathrm{dd}, J=8.9,2.6 \mathrm{~Hz}, 2 \mathrm{H}), 7.25-$ $7.20(\mathrm{~m}, 4 \mathrm{H}), 6.86(\mathrm{~d}, J=8.6 \mathrm{~Hz}, 2 \mathrm{H}), 6.62(\mathrm{~d}, J=8.8 \mathrm{~Hz}, 2 \mathrm{H})$, $5.87(\mathrm{~s}, 1 \mathrm{H}), 5.56(\mathrm{~s}, 1 \mathrm{H}), 4.59(\mathrm{~s}, 1 \mathrm{H}), 4.22(\mathrm{q}, J=7.1 \mathrm{~Hz}, 2 \mathrm{H})$, $1.16(\mathrm{t}, J=7.1 \mathrm{~Hz}, 3 \mathrm{H}) ;{ }^{13} \mathrm{C} \operatorname{NMR}\left(101 \mathrm{MHz}, \mathrm{CDCl}_{3}\right) \delta=169.2,167.6,143.6,139.7,133.1$, 132.6, 129.7, 129.5, 129.4, 125.6, 124.2, 123.5, 115.5, 113.7, 73.3, 68.4, 63.2, 14.0 ppm. 
Ethyl 1,3-bis(4-chlorophenyl)-4-((4-chlorophenyl)amino)-5-oxoimidazolidine-2-carboxylate $(7 e)^{3}$

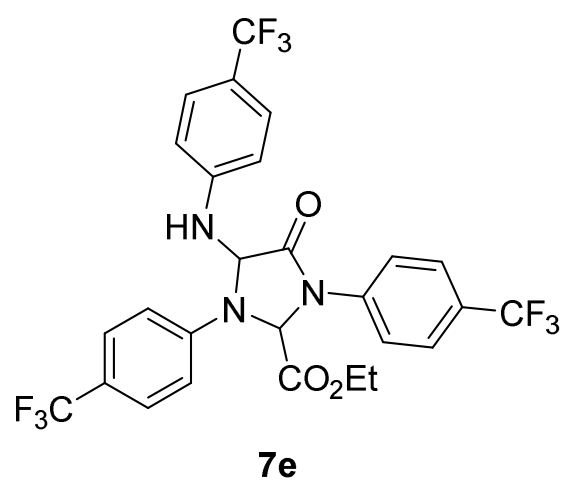

Colorless oil $\left(0.094 \mathrm{~g}, 78 \%\right.$ yield); $\mathrm{R}_{f}=0.5$ (silica, EtOAc:hexanes, 1.5:8.5); IR (film) $\mathrm{v}_{\max } 3414,1741,1618$, 1325, 1204 1170, 1056, $845 \mathrm{~cm}^{-1}$; ${ }^{1} \mathrm{H}$ NMR (400 MHz, $\left.\mathrm{CDCl}_{3}\right) \delta=7.81(\mathrm{~d}, J=8.5 \mathrm{~Hz}, 2 \mathrm{H}), 7.72(\mathrm{~d}, J=8.6 \mathrm{~Hz}, 2 \mathrm{H})$, $7.55(\mathrm{t}, J=8.6 \mathrm{~Hz}, 4 \mathrm{H}), 6.99(\mathrm{~d}, J=8.3 \mathrm{~Hz}, 2 \mathrm{H}), 6.78(\mathrm{~d}, J=$ $8.5 \mathrm{~Hz}, 2 \mathrm{H}), 6.06(\mathrm{~s}, 1 \mathrm{H}), 5.81(\mathrm{~d}, J=9.1 \mathrm{~Hz}, 1 \mathrm{H}), 4.97(\mathrm{~d}, J$ $=9.1 \mathrm{~Hz}, 1 \mathrm{H}), 4.27(\mathrm{q}, J=7.1 \mathrm{~Hz}, 2 \mathrm{H}), 1.20(\mathrm{t}, J=7.1 \mathrm{~Hz}, 3 \mathrm{H}) ;{ }^{13} \mathrm{C} \mathrm{NMR}\left(101 \mathrm{MHz}, \mathrm{CDCl}_{3}\right) \delta=$ $169.0,167.2,147.4,143.3,137.6,131.2,131.00,129.0,128.7,127.3,127.2,127.1,127.0$, $126.9,126.7,126.6,125.9,124.9,123.5,122.8,122.5,121.6,121.4,121.3,113.3,112.2,72.7$ 67.2, 63.6, $14.0 \mathrm{ppm}$.

diEthyl 4,4'-(2-(ethoxycarbonyl)-4-((4-(ethoxycarbonyl)phenyl)amino)-5-oxoimidazolidine1,3-diyl)dibenzoate (7f)

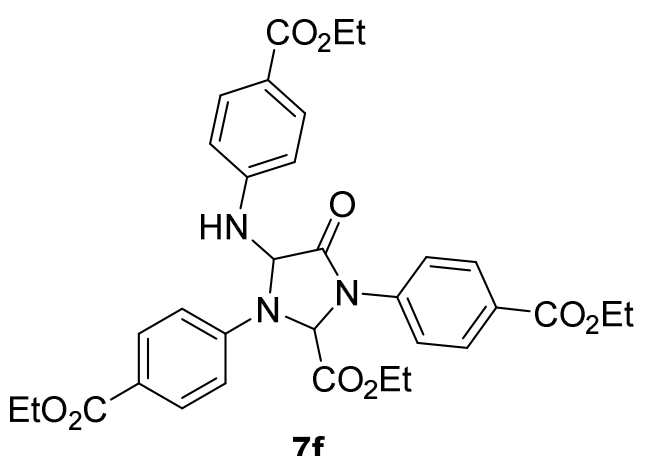

$7 f$
Yellow oil $\left(0.093 \mathrm{~g}, 75 \%\right.$ yield); $\mathrm{R}_{f}=0.5$ (silica, EtOAc:hexanes, 1.5:8.5); IR (film) $v_{\max } 3417,1730,1710$, 1606, 1516, 1273, 1187, 1020, $768 \mathrm{~cm}^{-1} ;{ }^{1} \mathrm{H}$ NMR $(400$ $\left.\mathrm{MHz}, \mathrm{CDCl}_{3}\right) \delta=8.18-8.07(\mathrm{~m}, 2 \mathrm{H}), 8.02-7.92(\mathrm{~m}$, $4 \mathrm{H}), 7.82-7.70(\mathrm{~m}, 2 \mathrm{H}), 6.95-6.89(\mathrm{~m}, 2 \mathrm{H}), 6.77-$ $6.66(\mathrm{~m}, 2 \mathrm{H}), 6.10(\mathrm{~s}, 1 \mathrm{H}), 5.85(\mathrm{~d}, \mathrm{~J}=9.1 \mathrm{~Hz}, 1 \mathrm{H}), 5.07$ $(\mathrm{d}, J=9.0 \mathrm{~Hz}, 1 \mathrm{H}), 4.44-4.20(\mathrm{~m}, 8 \mathrm{H}), 1.42-1.34(\mathrm{~m}, 9 \mathrm{H}), 1.20(\mathrm{t}, J=7.1 \mathrm{~Hz}, 3 \mathrm{H}) ;{ }^{13} \mathrm{C}$ NMR $\left(101 \mathrm{MHz}, \mathrm{CDCl}_{3}\right) \delta=169.2,167.1,166.5,166.0,165.5,148.8,144.2,138.5,131.7,130.9$, 128.6, 122.6, 121.3, 120.7, 112.8, 111.9, 72.6, 67.2, 63.5, 61.2, 60.7, 60.4, 14.4, 14.34, 14.31, 14.0 ppm; HRMS (m/z): [M + Na] ${ }^{+}$calcd for $\mathrm{C}_{33} \mathrm{H}_{35} \mathrm{~N}_{3} \mathrm{O}_{9} \mathrm{Na}^{+}$640.2265, Found 640.2261. 


\section{Ethyl 4-oxo-1,3-di-p-tolyl-5-(p-tolylamino)imidazolidine-2-carboxylate $\mathbf{( 7 g )}$}

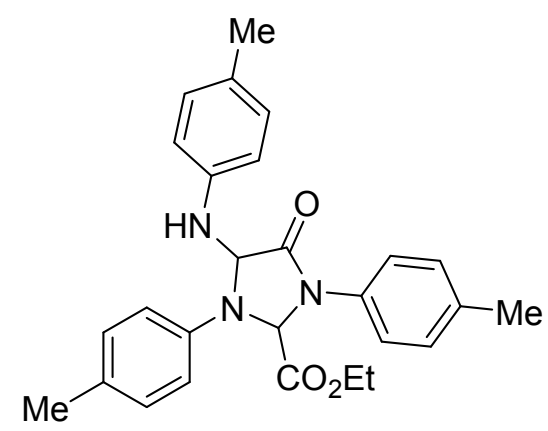

$7 \mathrm{~g}$
Pale yellow solid (0.043 g, $49 \%$ yield, m.p. $\left.94-96{ }^{\circ} \mathrm{C}\right) ; \mathrm{R}_{f}=$ 0.5 (silica, EtOAc:hexanes, 1:9); IR (film) $v_{\max } 3414,1731$, 1619, 1517, 1396, 1093, $806 \mathrm{~cm}^{-1} ;{ }^{1} \mathrm{H}$ NMR $(400 \mathrm{MHz}$, $\left.\mathrm{CDCl}_{3}\right) \delta=7.45(\mathrm{~d}, J=8.2 \mathrm{~Hz}, 2 \mathrm{H}), 7.21(\mathrm{~d}, J=8.2 \mathrm{~Hz}, 2 \mathrm{H})$, $7.07(\mathrm{~d}, J=8.0 \mathrm{~Hz}, 4 \mathrm{H}), 6.87(\mathrm{~d}, J=8.0 \mathrm{~Hz}, 2 \mathrm{H}), 6.65(\mathrm{~d}, J=$ $8.1 \mathrm{~Hz}, 2 \mathrm{H}), 5.86(\mathrm{~s}, 1 \mathrm{H}), 5.58(\mathrm{~d}, J=8.3 \mathrm{~Hz}, 1 \mathrm{H}), 4.41(\mathrm{~d}, J$

$=8.4 \mathrm{~Hz}, 1 \mathrm{H}), 4.18(\mathrm{q}, J=7.2 \mathrm{~Hz}, 2 \mathrm{H}), 2.36(\mathrm{~s}, 3 \mathrm{H}), 2.29(\mathrm{~s}, 3 \mathrm{H}), 2.26(\mathrm{~s}, 3 \mathrm{H}), 1.14(\mathrm{t}, J=7.1$ $\mathrm{Hz}, 3 \mathrm{H}) ;{ }^{13} \mathrm{C}$ NMR $\left(101 \mathrm{MHz}, \mathrm{CDCl}_{3}\right) \delta=169.6,168.3,143.2,139.4,136.8,132.2,130.2,129.8$, 129.3, 128.3, 122.6, 114.6, 112.5, 73.9, 69.2, 62.5, 21.0, 20.5, 20.3, 13.9 ppm; HRMS (m/z): [M $+\mathrm{Na}]^{+}$calcd for $\mathrm{C}_{27} \mathrm{H}_{29} \mathrm{~N}_{3} \mathrm{O}_{3} \mathrm{Na}^{+}$466.2101, Found 466.2098.

Ethyl 1,3-bis(3-fluorophenyl)-4-((3-fluorophenyl)amino)-5-oxoimidazolidine-2-carboxylate $(7 i)^{3}$

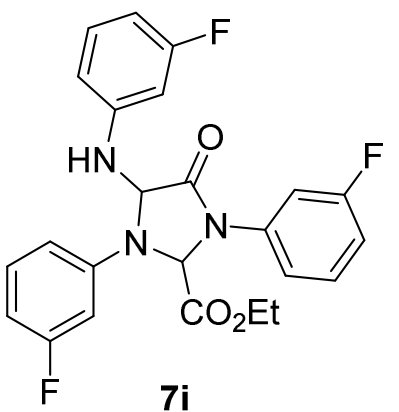

Pale yellow solid $\left(0.076 \mathrm{~g}, 83 \%\right.$ yield, m.p. $\left.101-103{ }^{\circ} \mathrm{C}\right) ; \mathrm{R}_{f}=0.3$ (silica, EtOAc:hexanes, 1.5:8.5); IR (film) $v_{\max } 3403,1746,1617,1495,1261$, 1015, 832, $764 \mathrm{~cm}^{-1} ;{ }^{1} \mathrm{H}$ NMR $\left(400 \mathrm{MHz}, \mathrm{CDCl}_{3}\right) \delta=7.52(\mathrm{~d}, J=10.4$ $\mathrm{Hz}, 1 \mathrm{H}), 7.43-7.33(\mathrm{~m}, 2 \mathrm{H}), 7.27-7.16(\mathrm{~m}, 2 \mathrm{H}), 6.99(\mathrm{t}, J=7.6 \mathrm{~Hz}$, $1 \mathrm{H}), 6.71-6.60(\mathrm{~m}, 3 \mathrm{H}), 6.56(\mathrm{td}, J=8.5,2.4 \mathrm{~Hz}, 1 \mathrm{H}), 6.50-6.46(\mathrm{~m}$, 2H), $5.89(\mathrm{~s}, 1 \mathrm{H}), 5.60(\mathrm{~d}, J=8.9 \mathrm{~Hz}, 1 \mathrm{H}), 4.73(\mathrm{~d}, J=9.0 \mathrm{~Hz}, 1 \mathrm{H}), 4.26(\mathrm{q}, J=7.1 \mathrm{~Hz}, 2 \mathrm{H})$, $1.19(\mathrm{t}, J=7.1 \mathrm{~Hz}, 3 \mathrm{H}) ;{ }^{13} \mathrm{C}$ NMR $\left(101 \mathrm{MHz}, \mathrm{CDCl}_{3}\right) \delta=169.1,167.4,165.1,165.0,164.1$, 162.7, 162.6, 161.6, 146.7, 146.6, 142.8, 142.7, 136.1, 136.0, 131.1, 131.0, 130.7, 130.6, 130.5, 116.8, 116.8, 113.9, 113.7, 109.8, 109.8, 109.5, 109.3, 108.2, 108.2, 107.2, 107.0, 106.1, 105.9, 101.4, 101.1, 100.3, 100.0, 73.0, 68.1, 63.2, 13.9 ppm; HRMS (m/z): $\left[\mathrm{M}+\mathrm{Na}^{+}\right.$calcd for $\mathrm{C}_{24} \mathrm{H}_{20} \mathrm{~F}_{3} \mathrm{~N}_{3} \mathrm{O}_{3} \mathrm{Na}^{+}$478.1349, Found 478.1345. 
carboxylate $(7 \mathrm{j})^{3}$

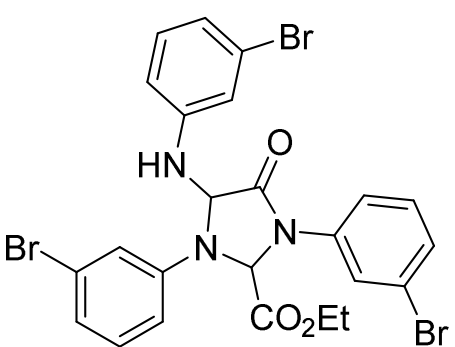

7j

Colorless oil $\left(0.101 \mathrm{~g}, 80 \%\right.$ yield); $\mathrm{R}_{f}=0.3$ (silica, EtOAc:hexanes, 1.5:8.5); IR (film) $v_{\max } 3418,1738,1619,1481,1201,1015,766 \mathrm{~cm}^{-}$ 1; ${ }^{1} \mathrm{H}$ NMR $\left(400 \mathrm{MHz}, \mathrm{CDCl}_{3}\right) \delta=7.83(\mathrm{t}, J=2.0 \mathrm{~Hz}, 1 \mathrm{H}), 7.61-$ $7.52(\mathrm{~m}, 1 \mathrm{H}), 7.43(\mathrm{dt}, J=8.2,1.2 \mathrm{~Hz}, 1 \mathrm{H}), 7.30(\mathrm{t}, J=8.1 \mathrm{~Hz}, 1 \mathrm{H})$, $7.16-7.08(\mathrm{~m}, 3 \mathrm{H}), 7.07-7.03(\mathrm{~m}, 1 \mathrm{H}), 6.99(\mathrm{dd}, J=7.9,1.8 \mathrm{~Hz}$, $1 \mathrm{H}), 6.92-6.83(\mathrm{~m}, 2 \mathrm{H}), 6.61(\mathrm{dd}, J=8.2,2.4 \mathrm{~Hz}, 1 \mathrm{H}), 5.89(\mathrm{~s}, 1 \mathrm{H}), 5.62(\mathrm{~d}, J=9.1 \mathrm{~Hz}, 1 \mathrm{H})$, $4.70(\mathrm{~d}, J=9.1 \mathrm{~Hz}, 1 \mathrm{H}), 4.32-4.22(\mathrm{~m}, 2 \mathrm{H}), 1.22(\mathrm{t}, J=7.1 \mathrm{~Hz}, 3 \mathrm{H}) ;{ }^{13} \mathrm{C}$ NMR $(101 \mathrm{MHz}$, $\left.\mathrm{CDCl}_{3}\right) \delta=169.2,167.3,146.3,142.2,135.8,131.1,130.8,130.6,130.1,125.0,123.7,123.4$ $123.4,123.0,122.5,120.3,117.2,115.5,112.8,111.5,72.9,67.8,63.4,14.0$ ppm.

Ethyl

4-oxo-1,3-bis(3-(trifluoromethyl)phenyl)-5-((3(trifluoromethyl)phenyl)amino)imidazolidine-2-carboxylate $(7 \mathrm{k})^{3}$

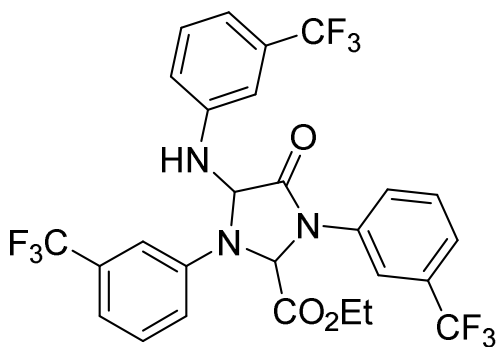

$7 k$
Pale yellow solid (0.098 g, $81 \%$ yield, m.p. $\left.119-121^{\circ} \mathrm{C}\right) ; \mathrm{R}_{f}=0.4$ (silica, EtOAc:hexanes, 1.5:8.5); IR (film) $\mathrm{V}_{\max }$ 3413, 1744, 1618, $1377,1126,861,788 \mathrm{~cm}^{-1} ;{ }^{1} \mathrm{H} \operatorname{NMR}\left(400 \mathrm{MHz} \mathrm{CDCl}_{3}\right) \delta=7.96-$ $7.89(\mathrm{~m}, 2 \mathrm{H}), 7.62-7.54(\mathrm{~m}, 2 \mathrm{H}), 7.39(\mathrm{td}, J=7.9,4.7 \mathrm{~Hz}, 2 \mathrm{H})$, $7.20(\mathrm{~d}, J=7.8 \mathrm{~Hz}, 1 \mathrm{H}), 7.17-7.11(\mathrm{~m}, 3 \mathrm{H}), 6.97(\mathrm{t}, J=2.0 \mathrm{~Hz}$,

1H), 6.87 (dd, J = 8.4, $2.5 \mathrm{~Hz}, 1 \mathrm{H}), 6.04(\mathrm{~s}, 1 \mathrm{H}), 5.77$ (d, J = 9.2 Hz, 1H), 4.91 (d, J = 9.2 Hz, $1 \mathrm{H}), 4.32-4.22(\mathrm{~m}, 2 \mathrm{H}), 1.21(\mathrm{t}, J=7.1 \mathrm{~Hz}, 3 \mathrm{H}) ;{ }^{13} \mathrm{C} \operatorname{NMR}\left(101 \mathrm{MHz}, \mathrm{CDCl}_{3}\right) \delta=169.4,167.4$, $145.2,141.1,135.3,132.3,132.1,132.0,131.8,131.7,131.6,130.4,130.2,130.1,125.4,125.1$, $125.0,124.8,123.6,123.5,123.5,122.7,122.4,122.1,121.8,118.5,118.4,117.2,117.1,117.0$ 116.3, 116.2, 116.0, 111.0, 110.9, 109.1, 109.0, 72.8, 67.8, 63.6, 13.8 ppm. 


\section{References}

1 (a) Sato, Y.; Tamura, T.; Kinbara, A.; Morib, M. Adv. Synth. Catal. 2007, 349, 647. (b) Pena, D.; Cobas, A.; Pérez, D.; Guitián, E. Synthesis 2002, 1454.

2 (a) Shou, W.-G.; Yang, Y.-Y.; Wang, Y.-G. J. Org. Chem. 2006, 71, 9241. (b) Castillo, J.-C.; Quiroga, J.; Abonia, R.; Rodriguez, J.; Coquerel, Y. Org. Lett. 2015, 17, 3374. (c) Castillo, J.-C.; Quiroga, J.; Abonia, R.; Rodriguez, J.; Coquerel, Y. J. Org. Chem. 2015, 80, 9767.

3 Ma, G.-N.; Wang, F.-J.; Gao, J.; Shi, M. Chem. Commun. 2008, 4998.

4 Bhojgude, S. S.; Bhunia, A.; Gonnade, R. G.; Biju, A. T. Org. Lett. 2014, 16, 676. 


\section{Spectra}

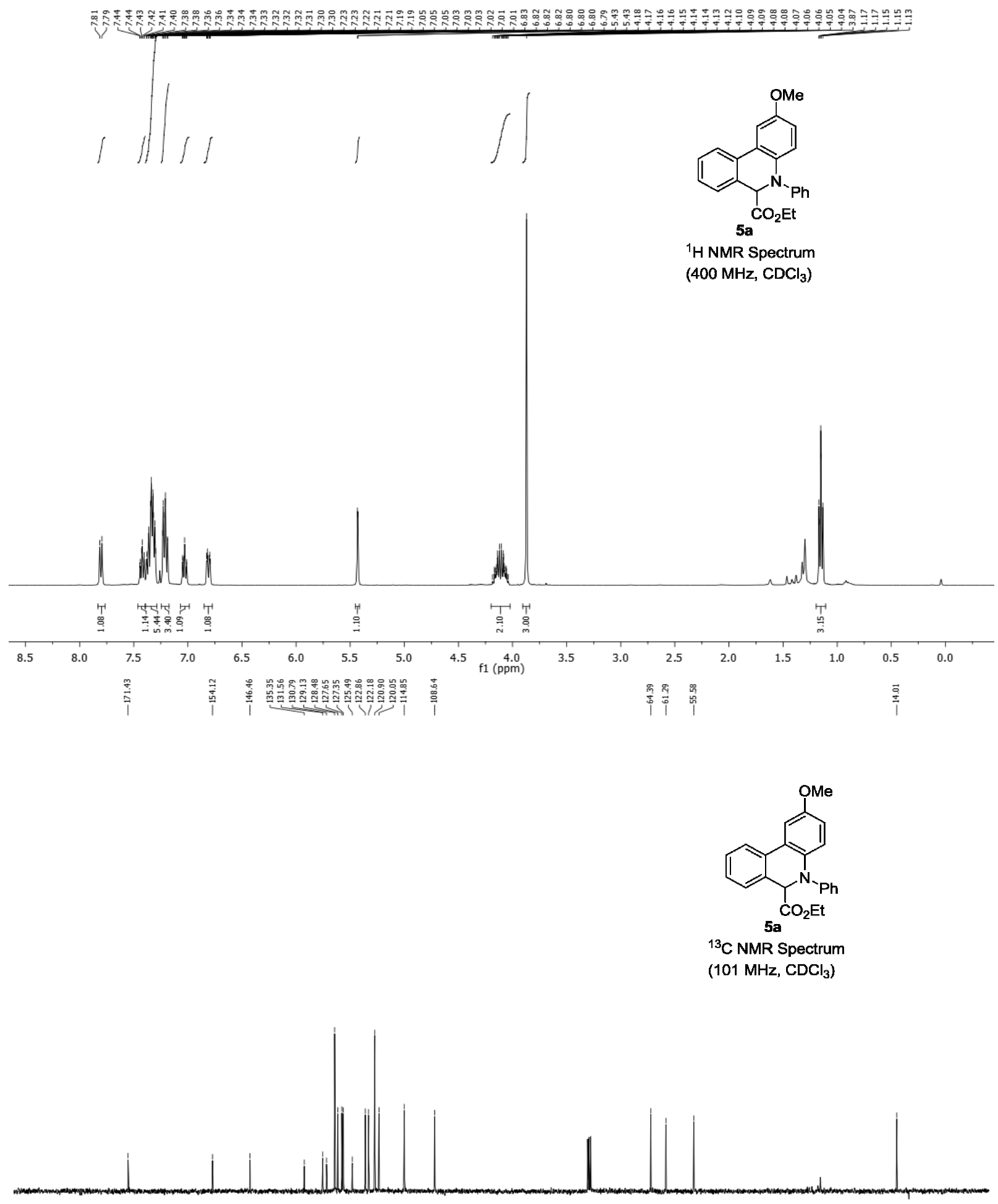



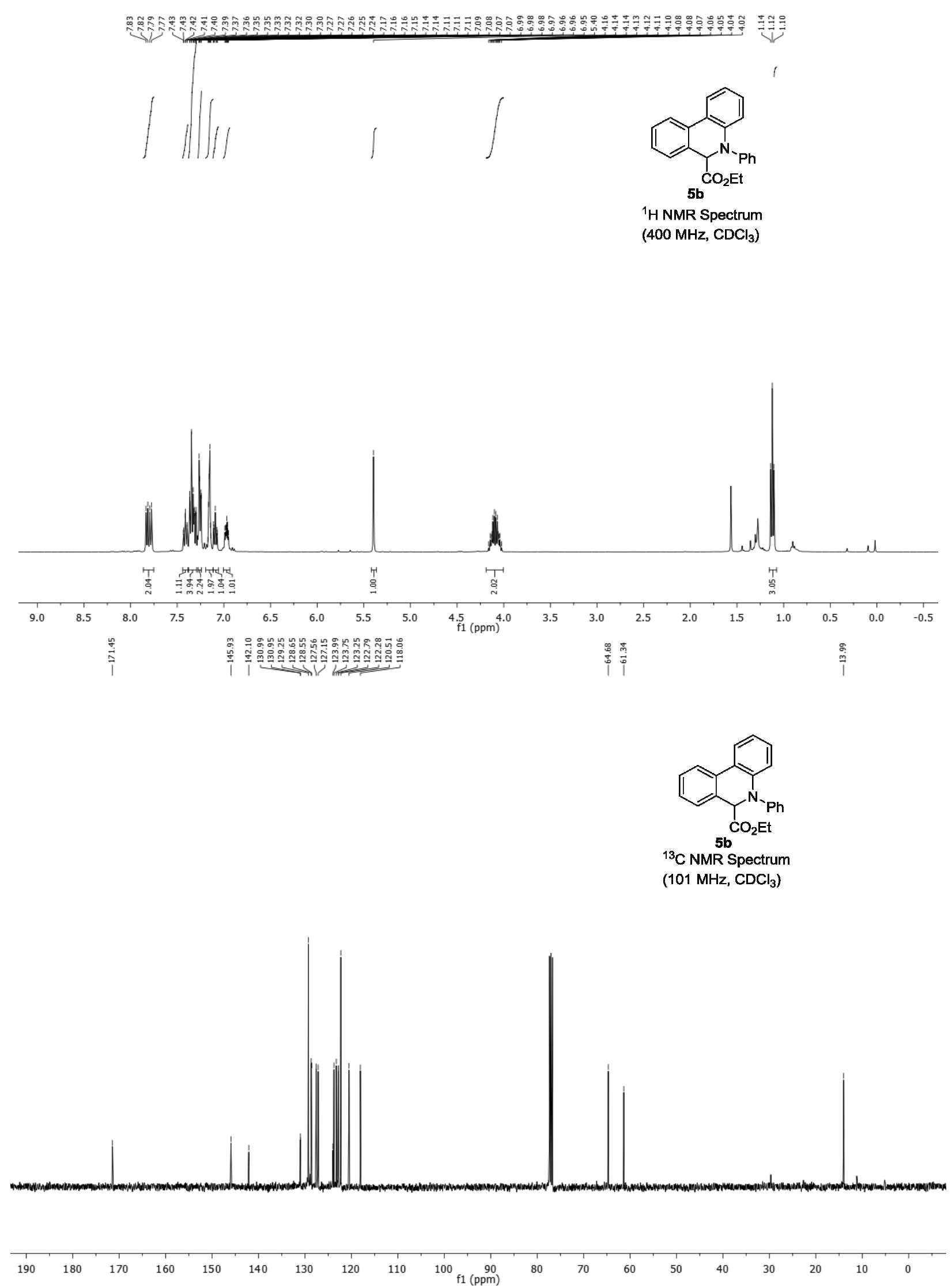

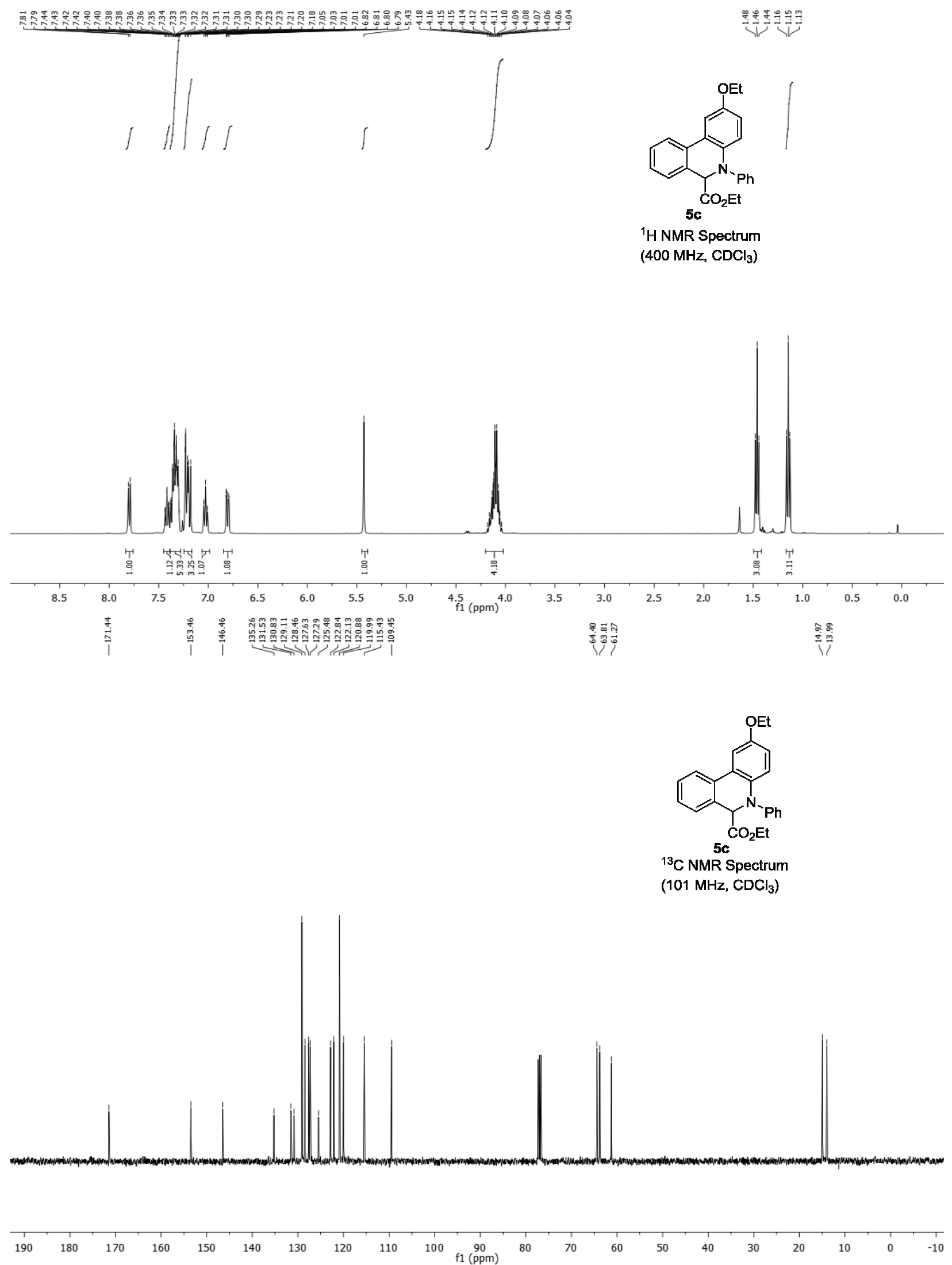


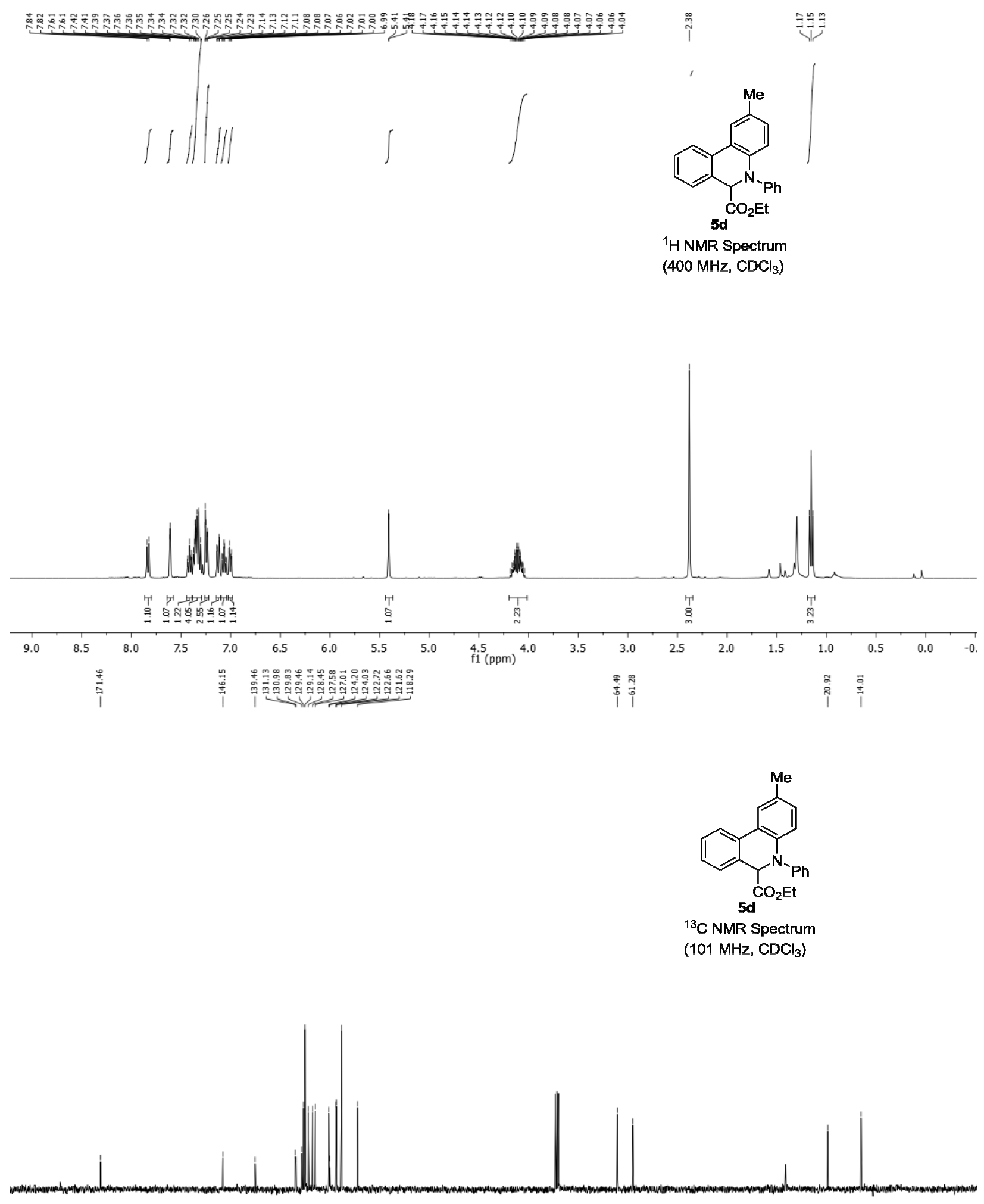

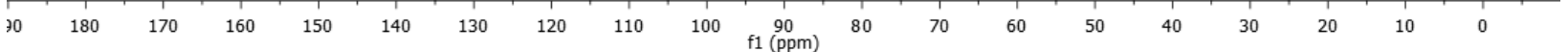



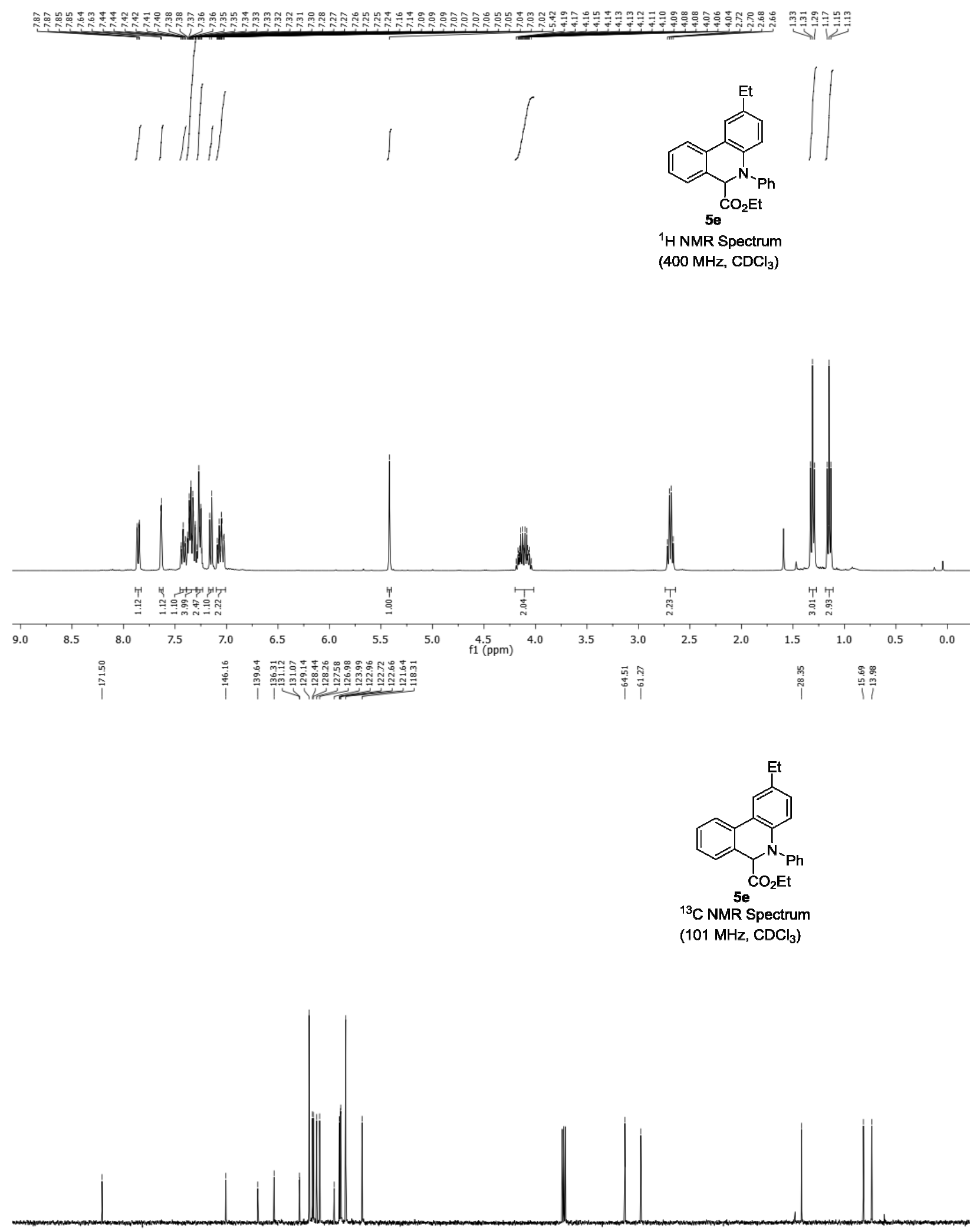

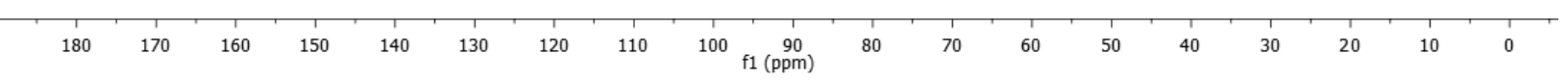



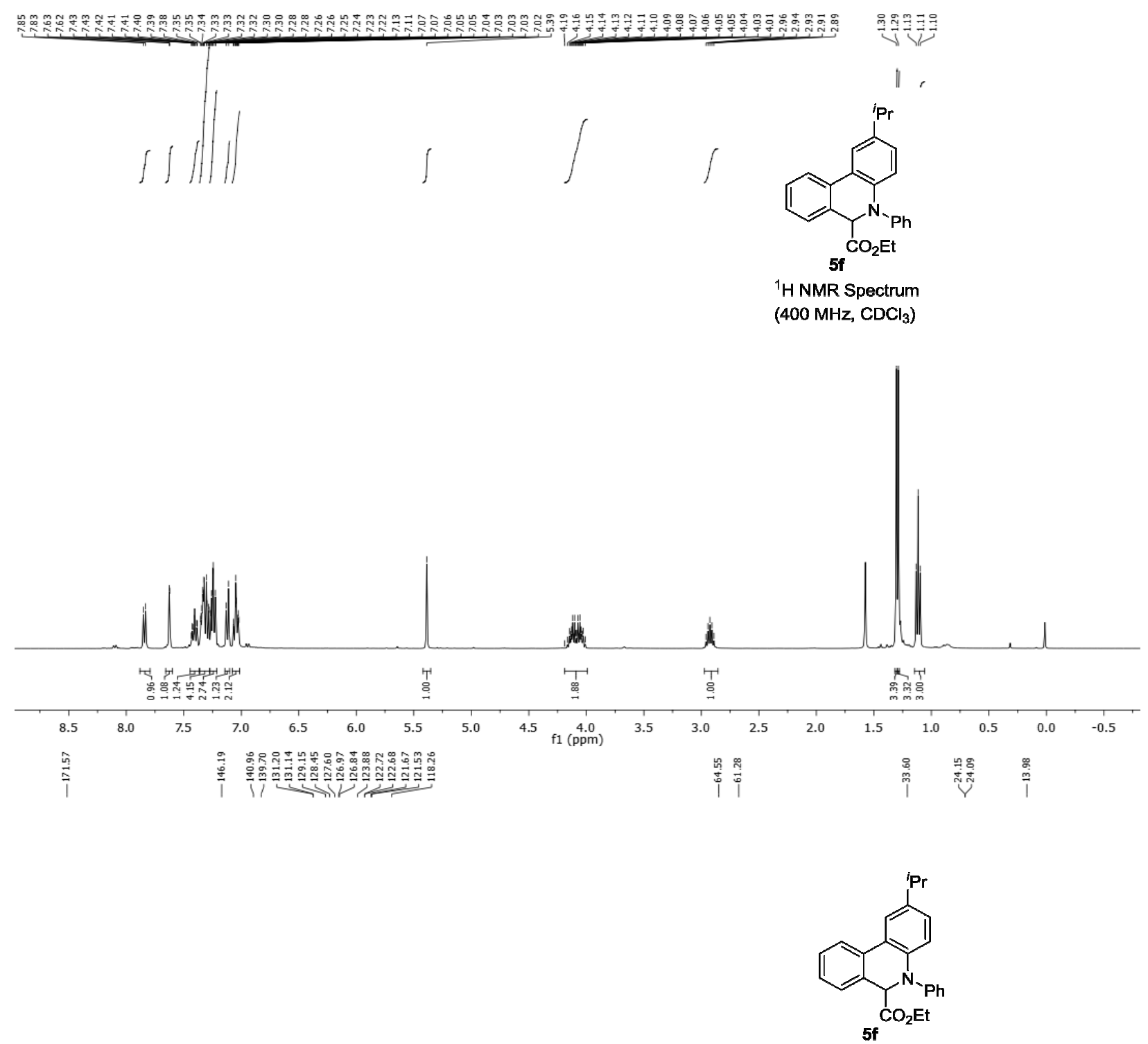

${ }^{13} \mathrm{C}$ NMR Spectrum (101 MHz, $\mathrm{CDCl}_{3}$ )
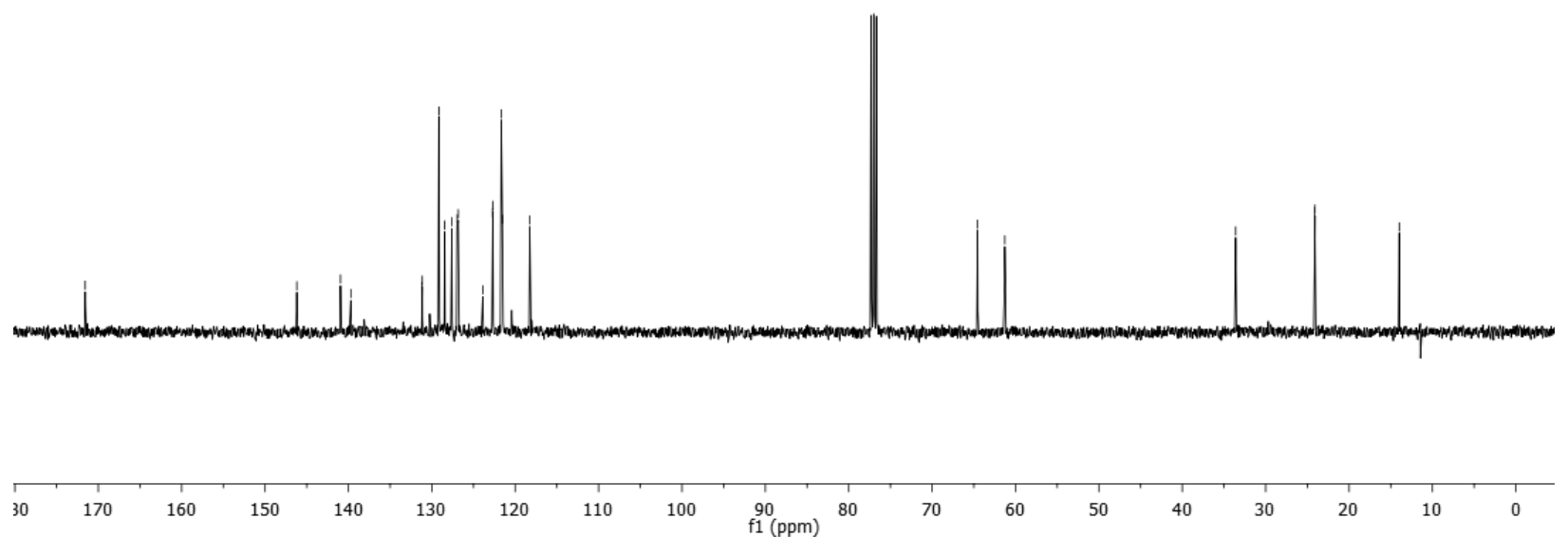

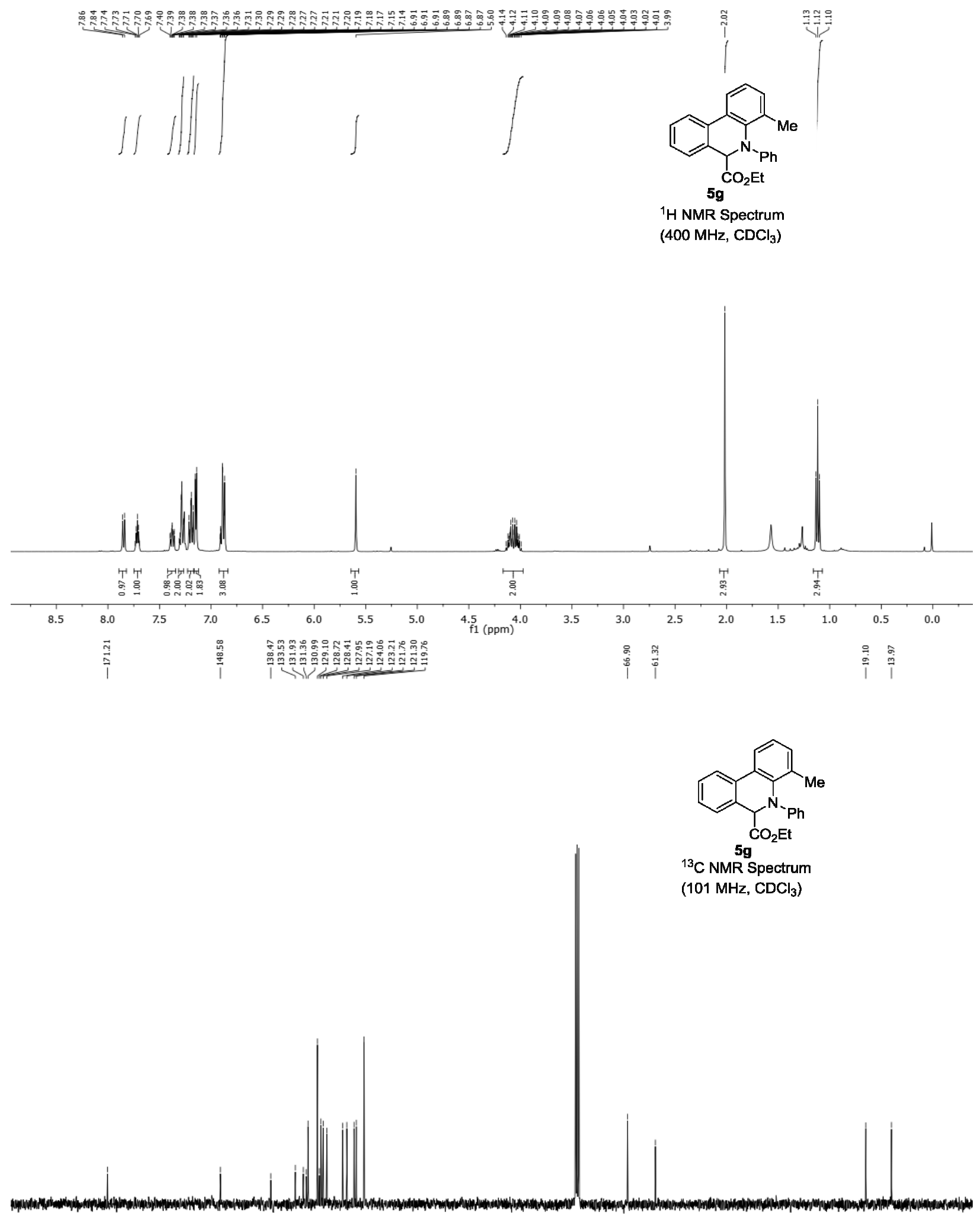

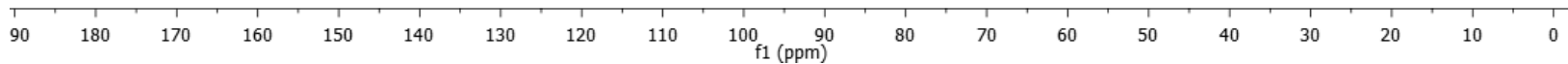




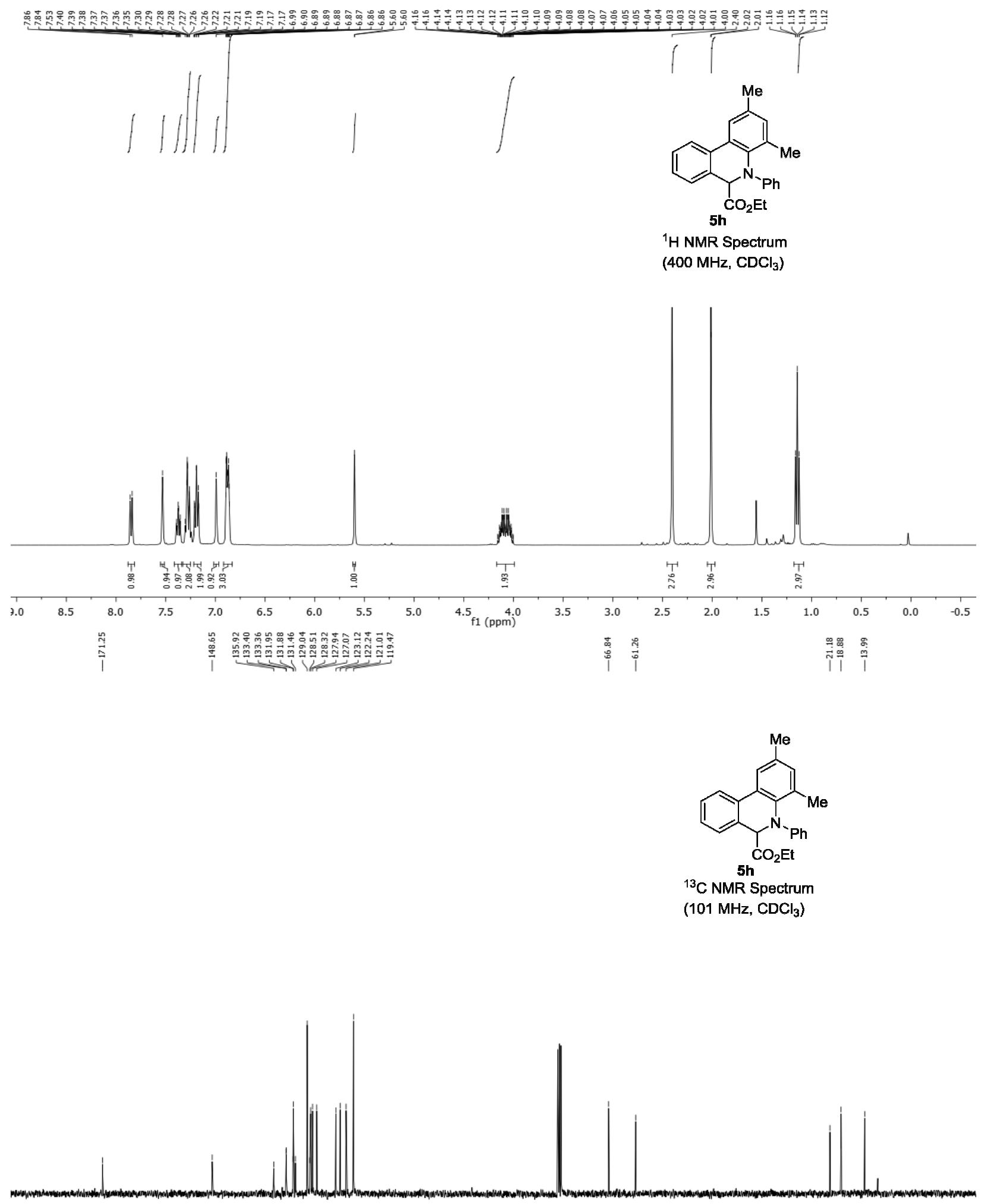

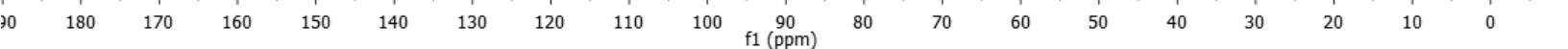



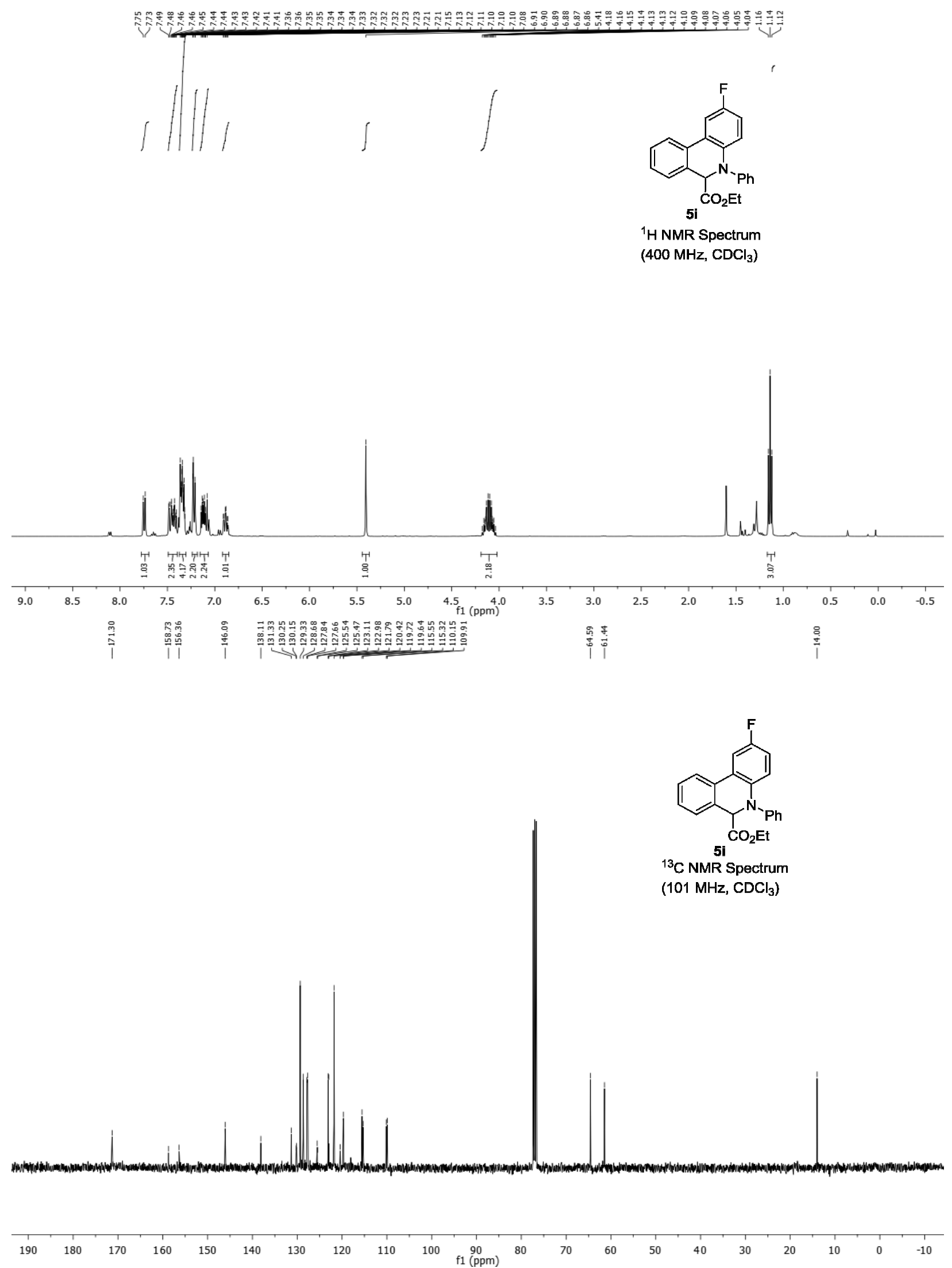

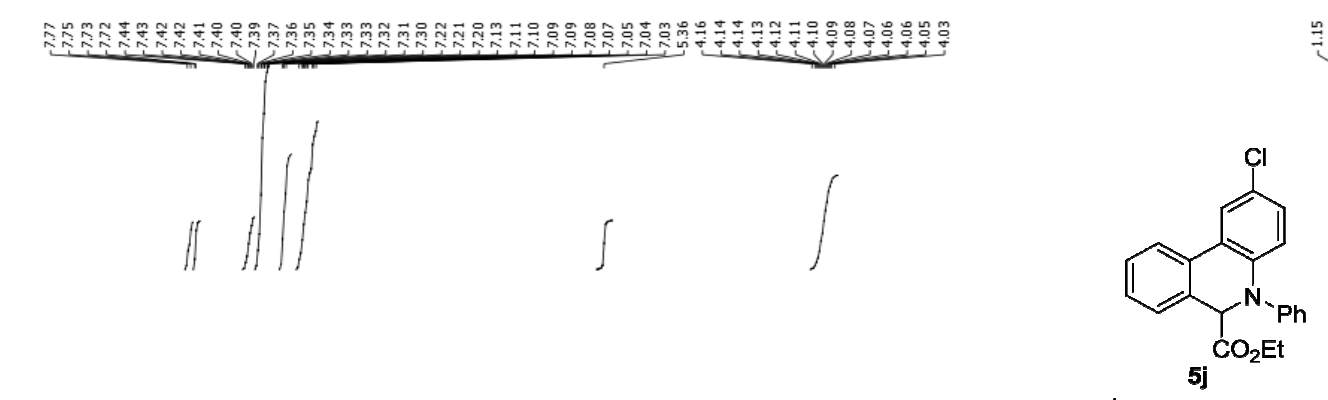

${ }^{1} \mathrm{H}$ NMR Spectrum

(400 MHz, $\mathrm{CDCl}_{3}$ )
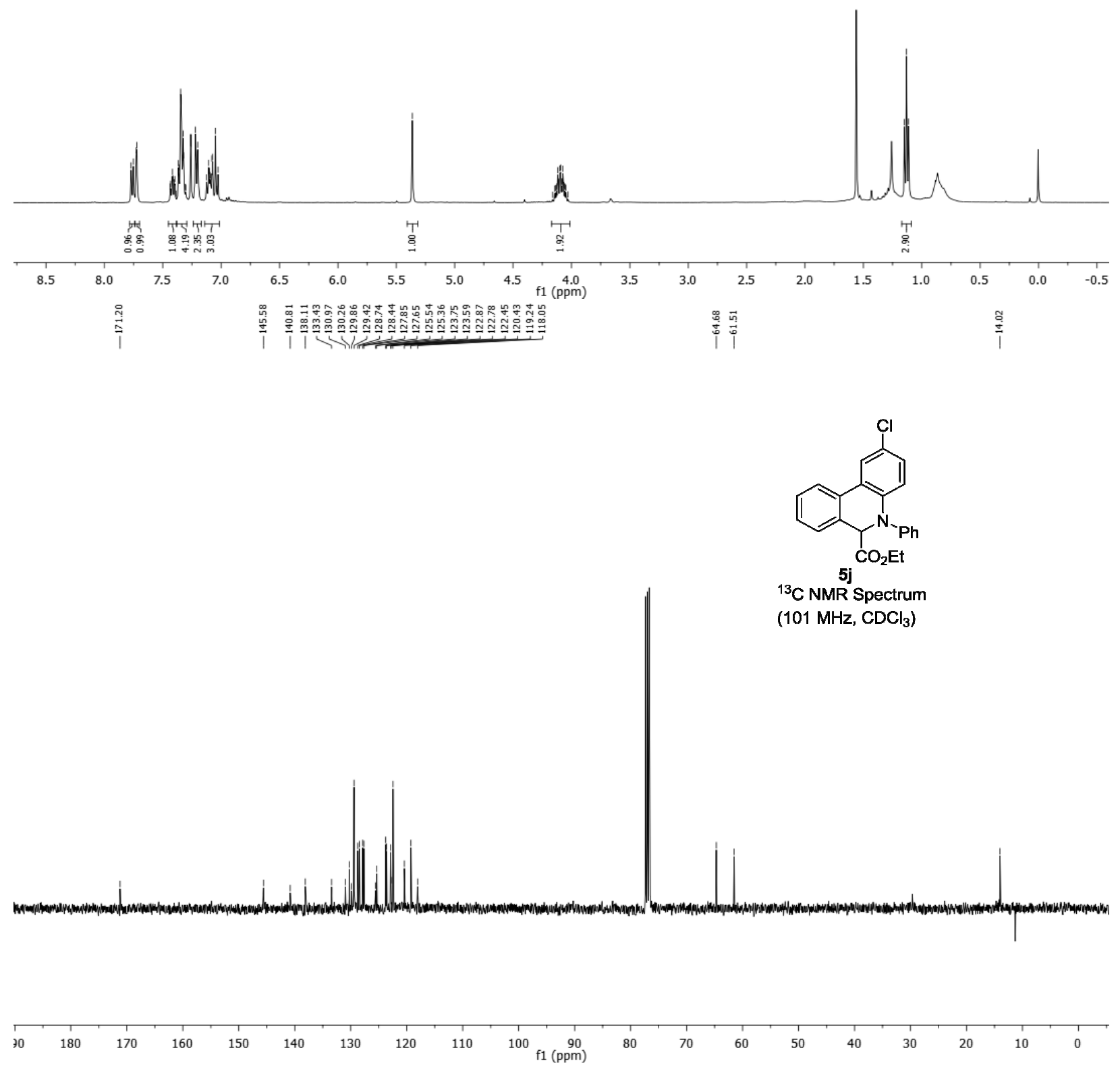

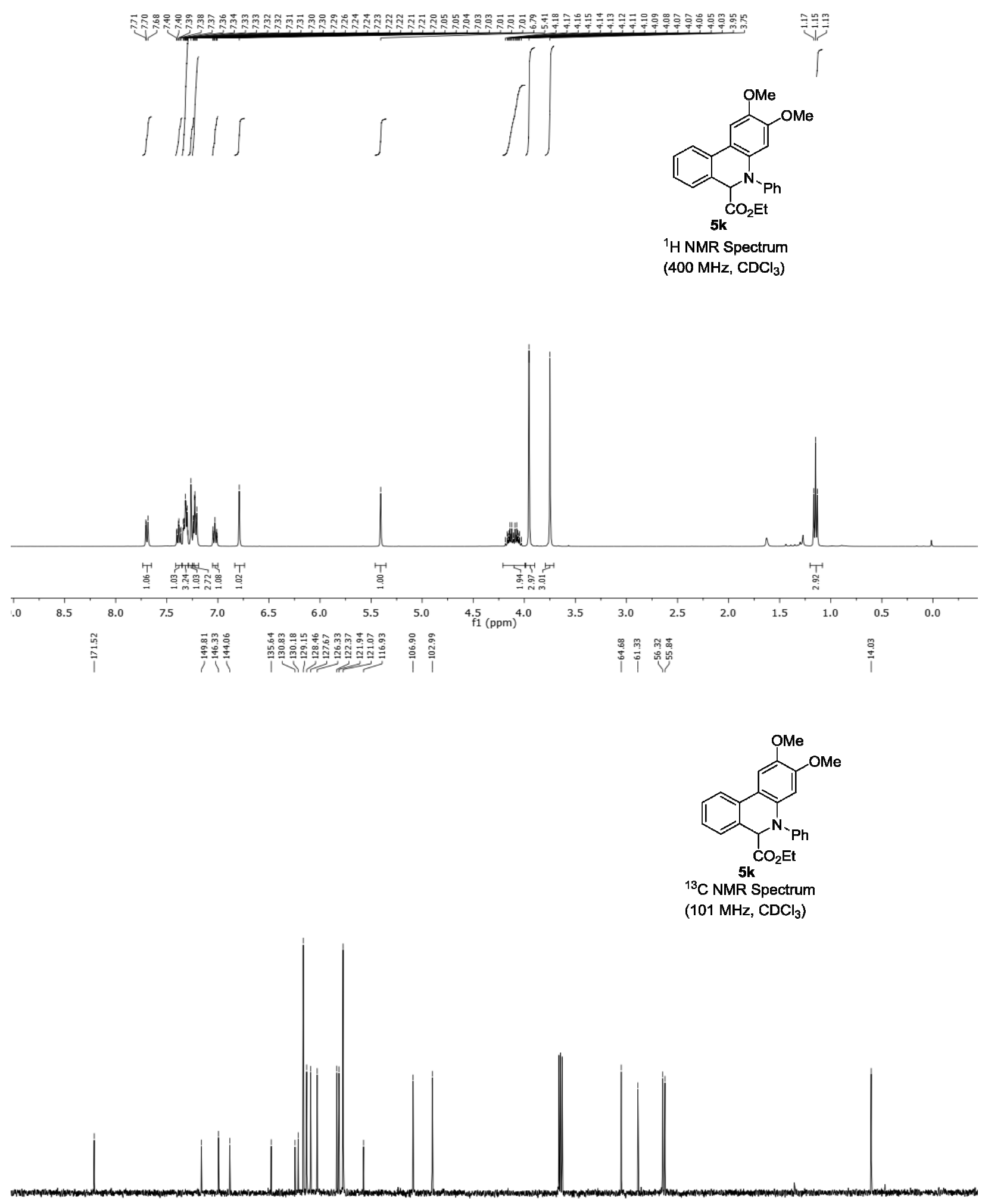
<smiles>CCOC(=O)C1c2ccccc2-c2cc(C)c(C)cc2N1c1ccccc1</smiles>

$5 \mathrm{I}: 5 I^{\prime}=1.2: 1$

${ }^{1} \mathrm{H}$ NMR Spectrum ( $400 \mathrm{MHz}, \mathrm{CDCl}_{3}$ )

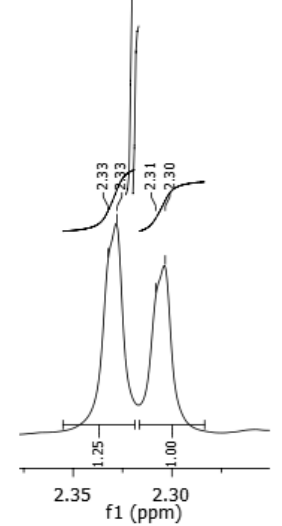

$M$

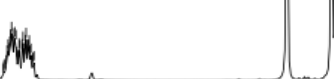

萑

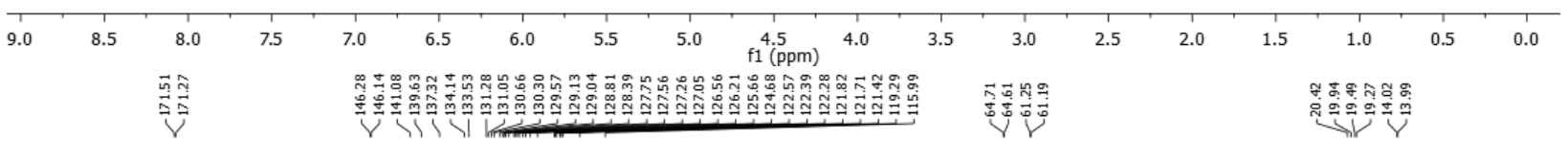<smiles>CCOC(=O)C1c2ccccc2-c2cc(C)c(C)cc2N1c1ccccc1</smiles>

$51: 5 l^{\prime}=1.2: 1$

${ }^{13} \mathrm{C}$ NMR Spectrum (101 $\mathrm{MHz}, \mathrm{CDCl}_{3}$ )

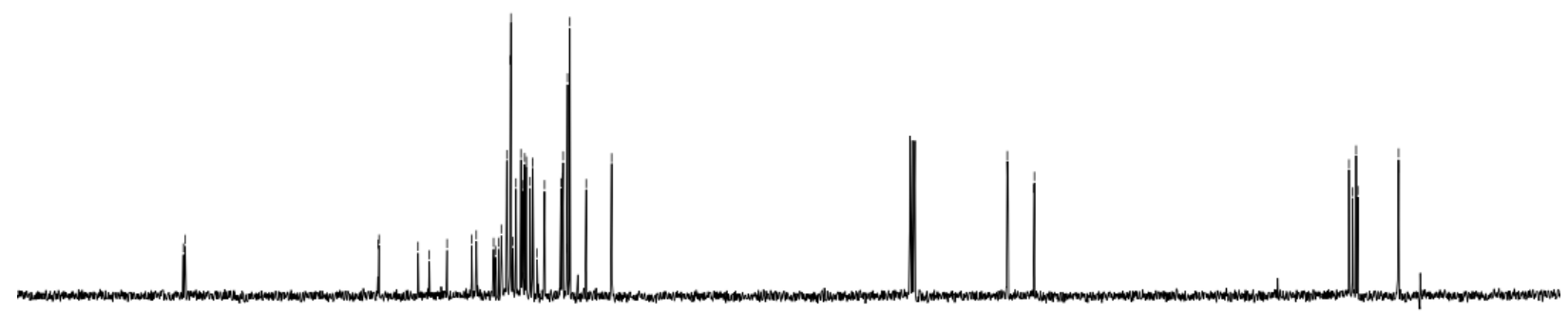



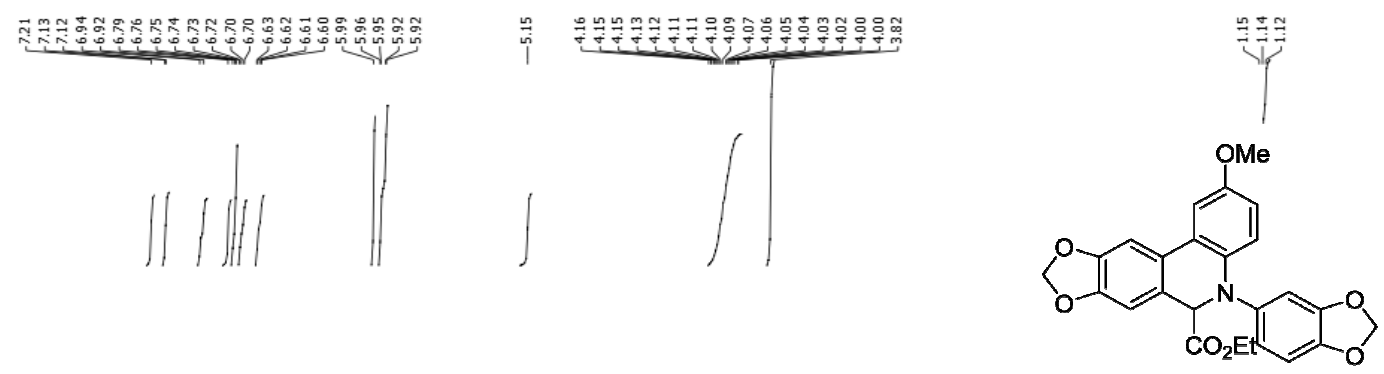

$5 \mathrm{~m}$

${ }^{1} \mathrm{H}$ NMR Spectrum

( $400 \mathrm{MHz}, \mathrm{CDCl}_{3}$ )
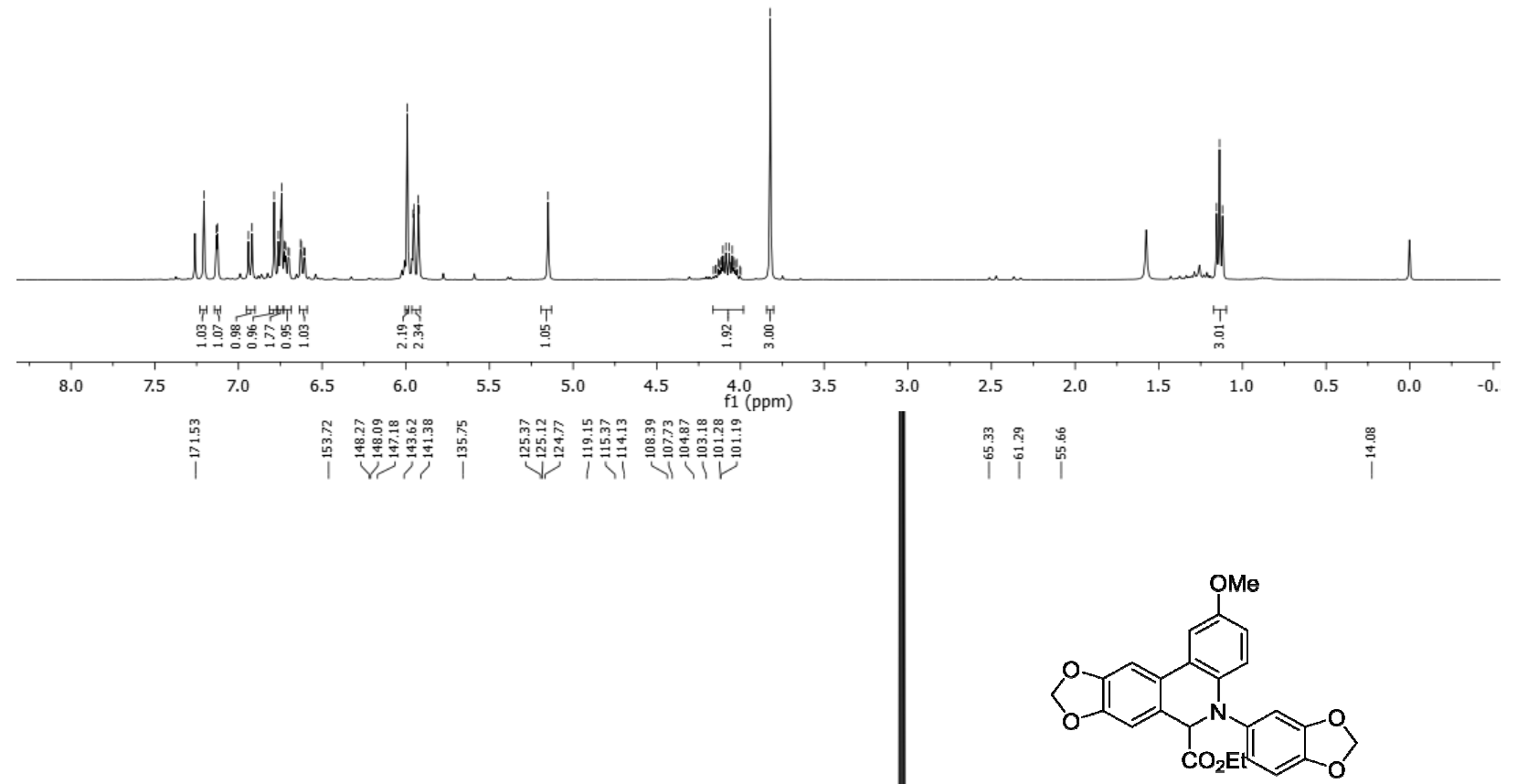

$5 \mathrm{~m}$

${ }^{13} \mathrm{C}$ NMR Spectrum (101 $\mathrm{MHz}, \mathrm{CDCl}_{3}$ )

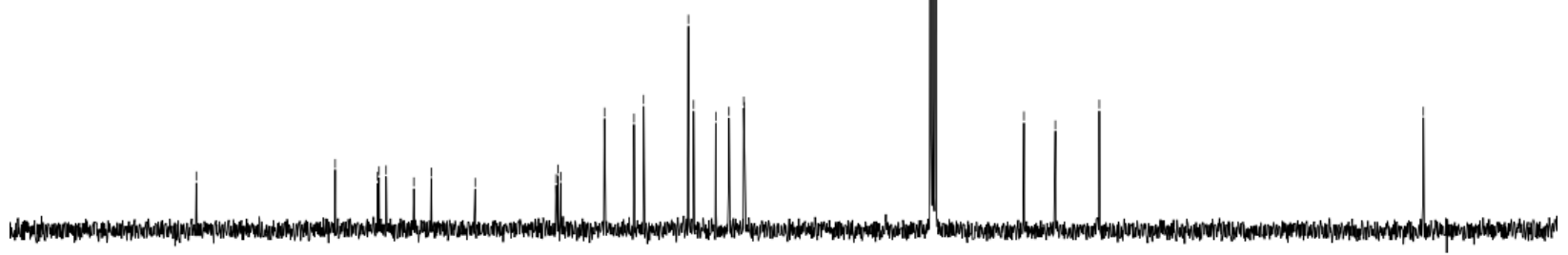

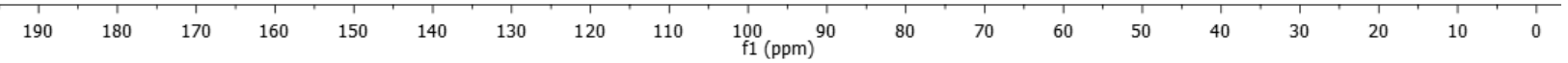



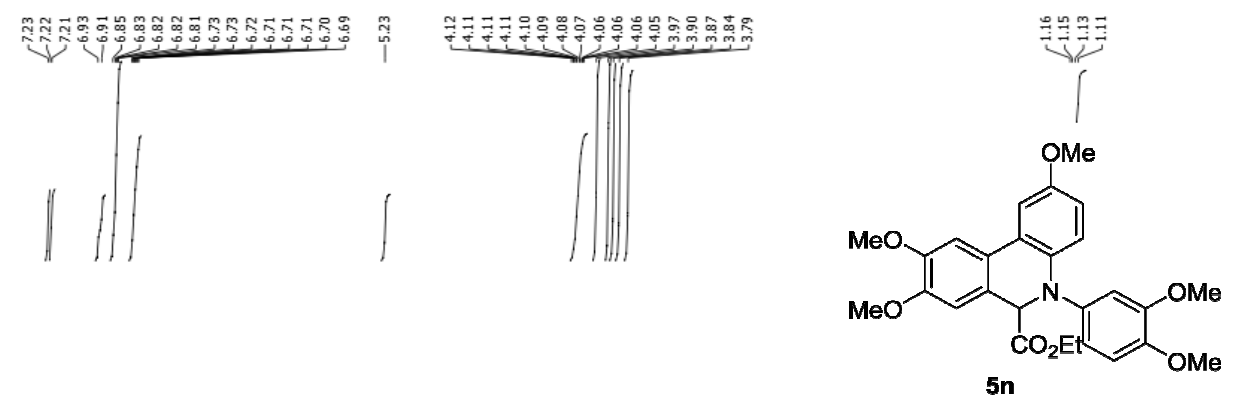

${ }^{1} \mathrm{H}$ NMR Spectrum

$\left(400 \mathrm{MHz}, \mathrm{CDCl}_{3}\right.$ )
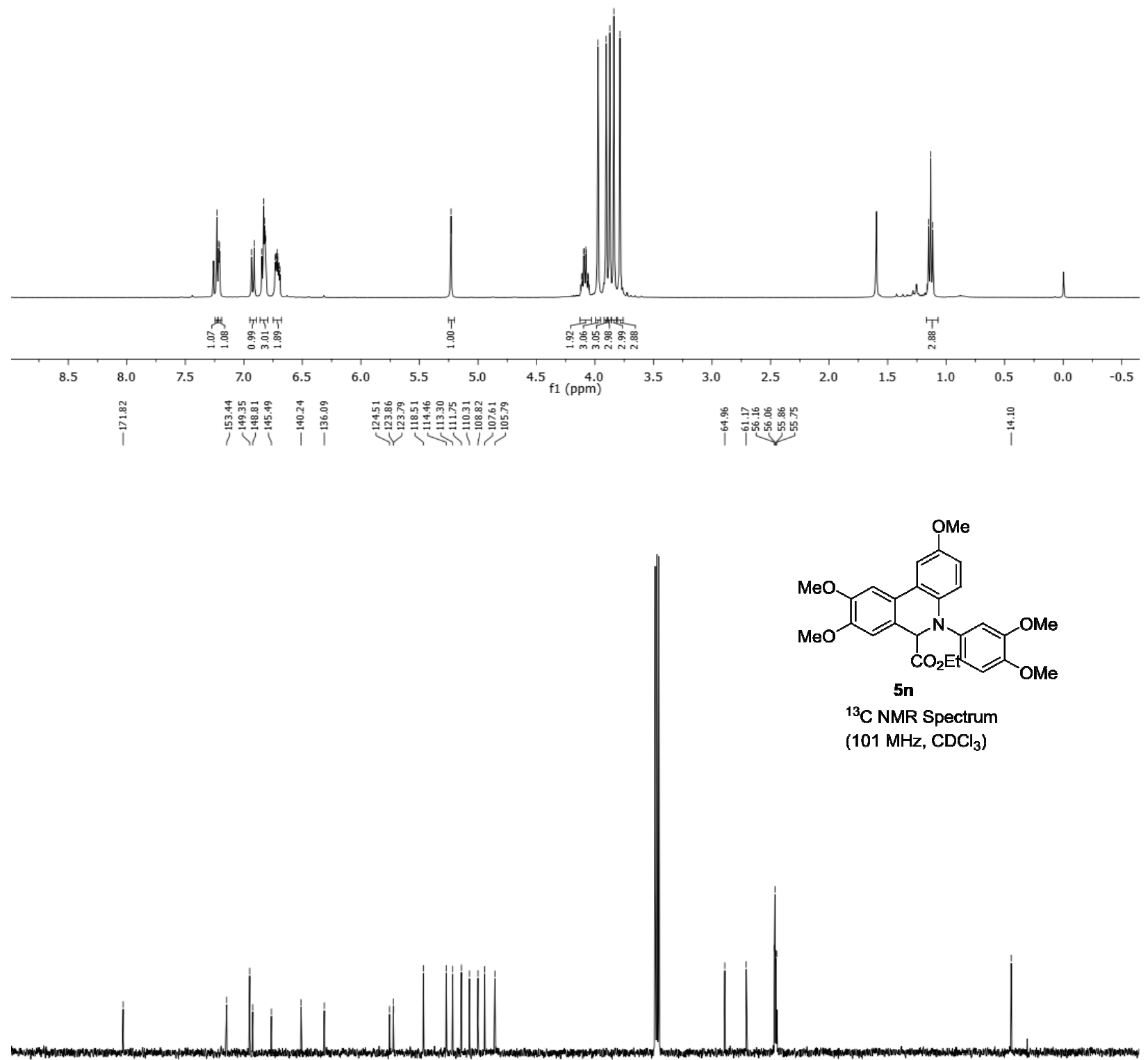

19
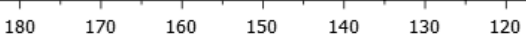

$110 \quad 100 \quad \begin{gathered}90 \\ \text { f1 }\end{gathered}$

80

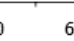




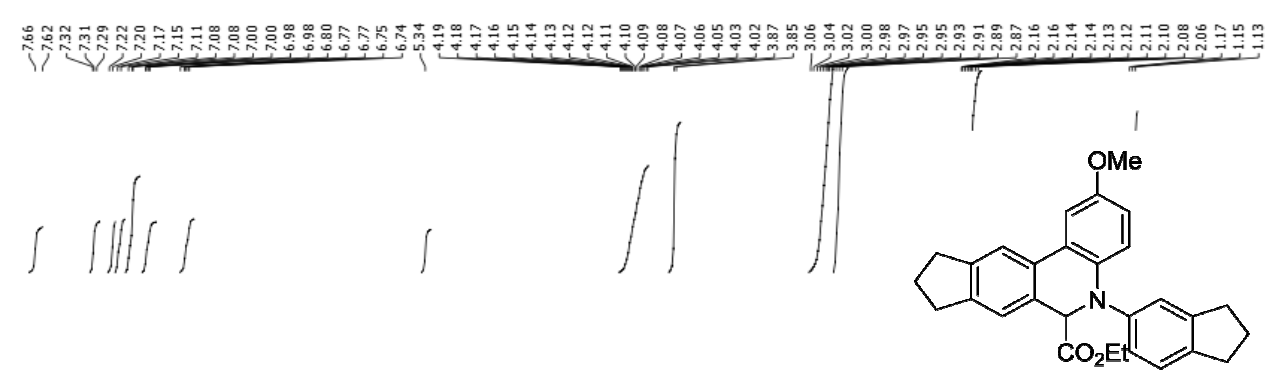

50

${ }^{1} \mathrm{H}$ NMR Spectrum

$\left(400 \mathrm{MHz}, \mathrm{CDCl}_{3}\right)$
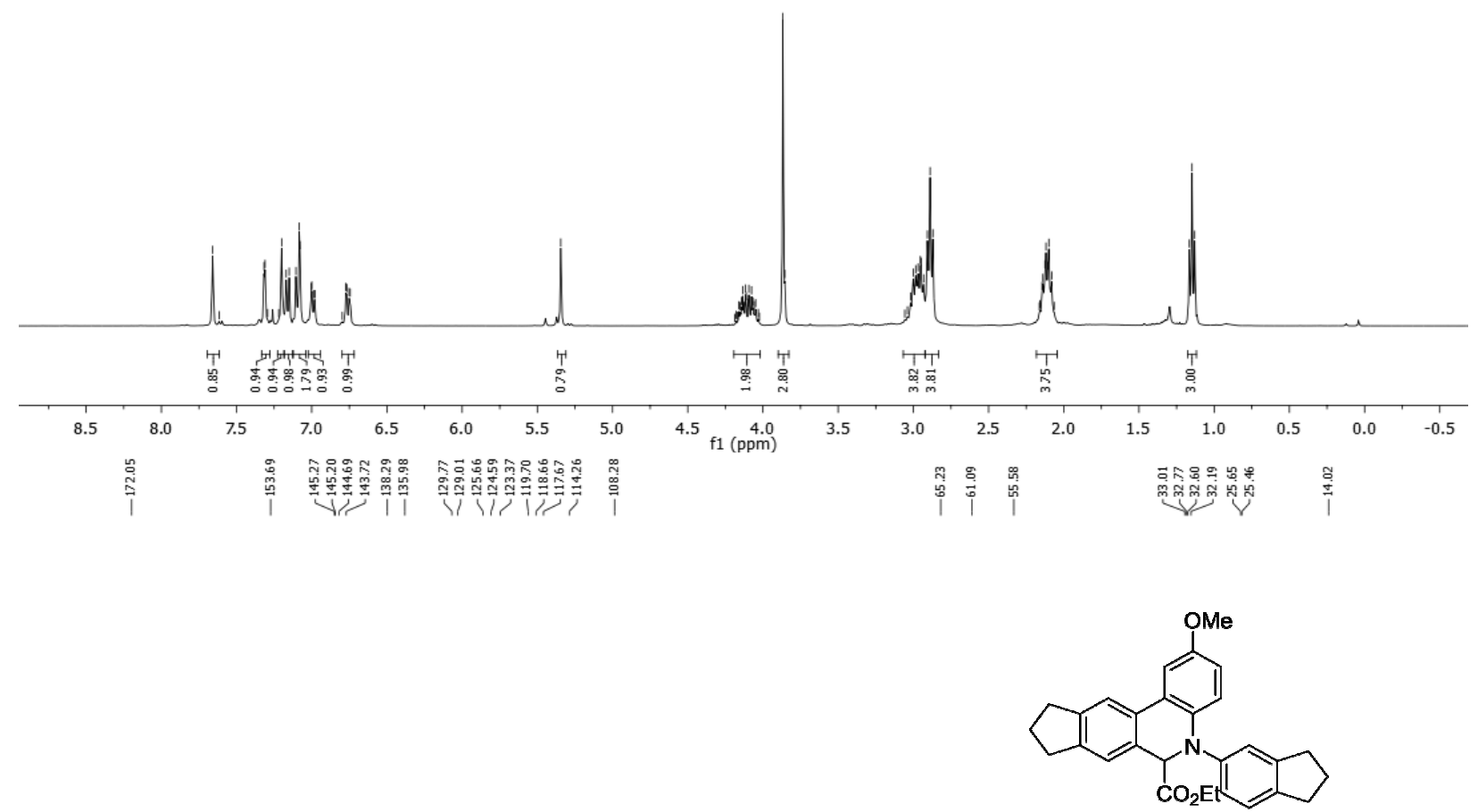

50

${ }^{13} \mathrm{C}$ NMR Spectrum

(101 MHz, $\mathrm{CDCl}_{3}$ )

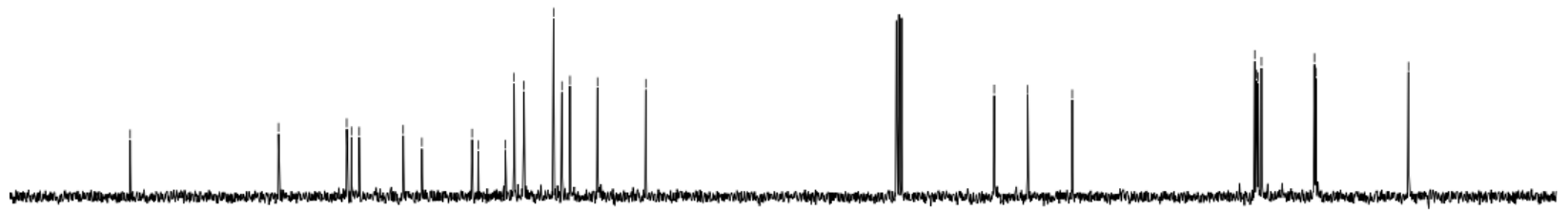

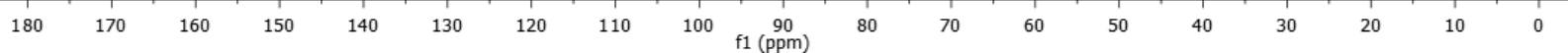




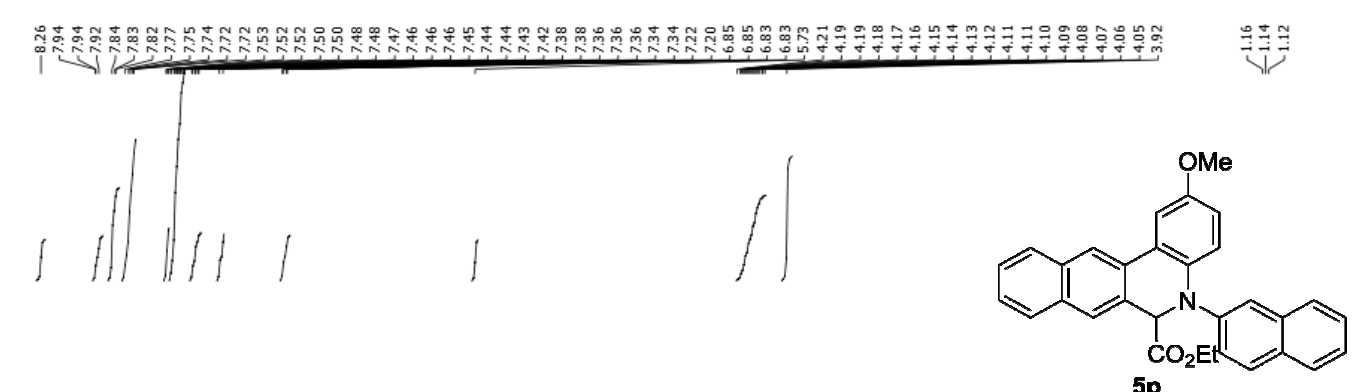

${ }^{1} \mathrm{H}$ NMR Spectrum (400 MHz, $\mathrm{CDCl}_{3}$ )
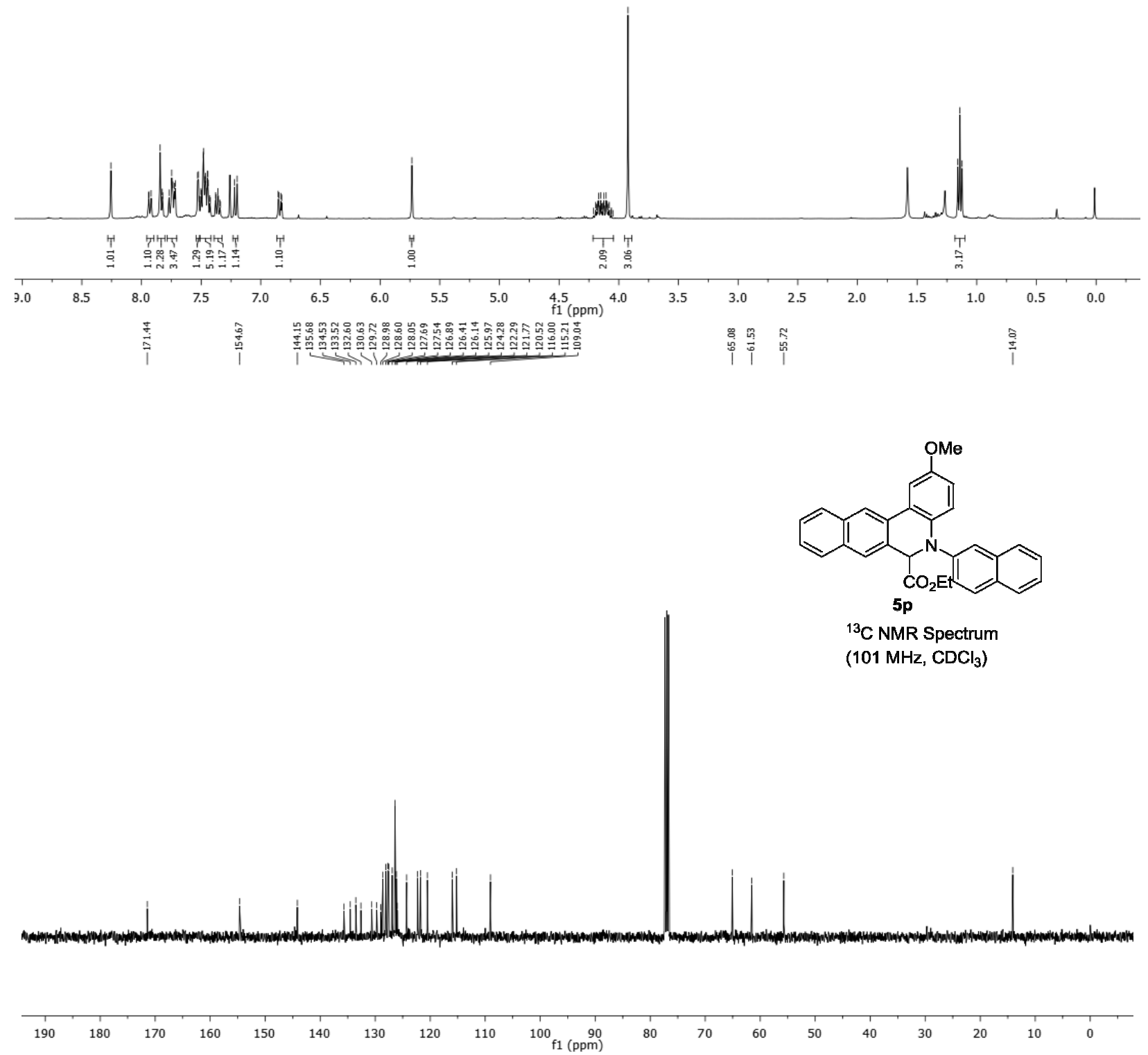

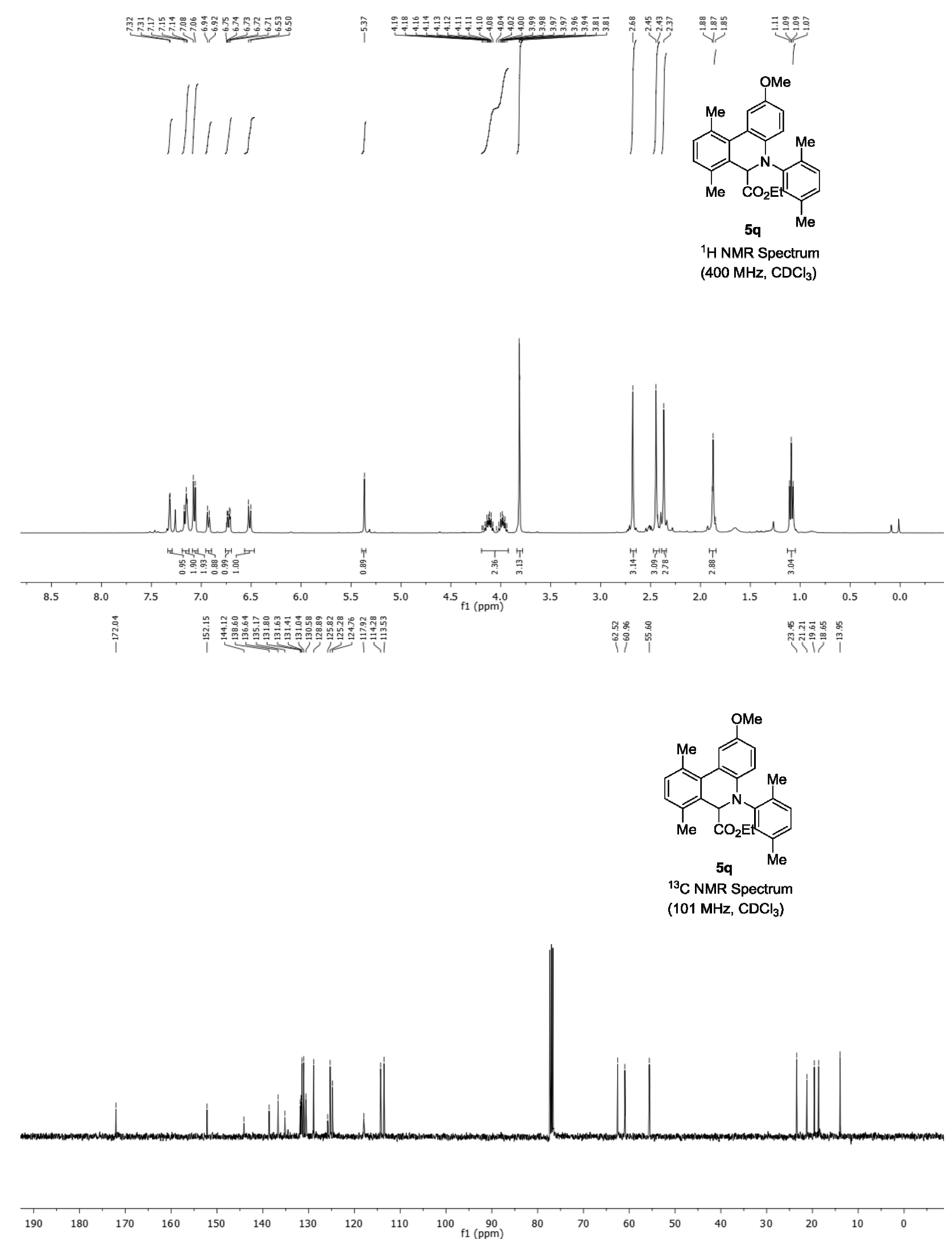

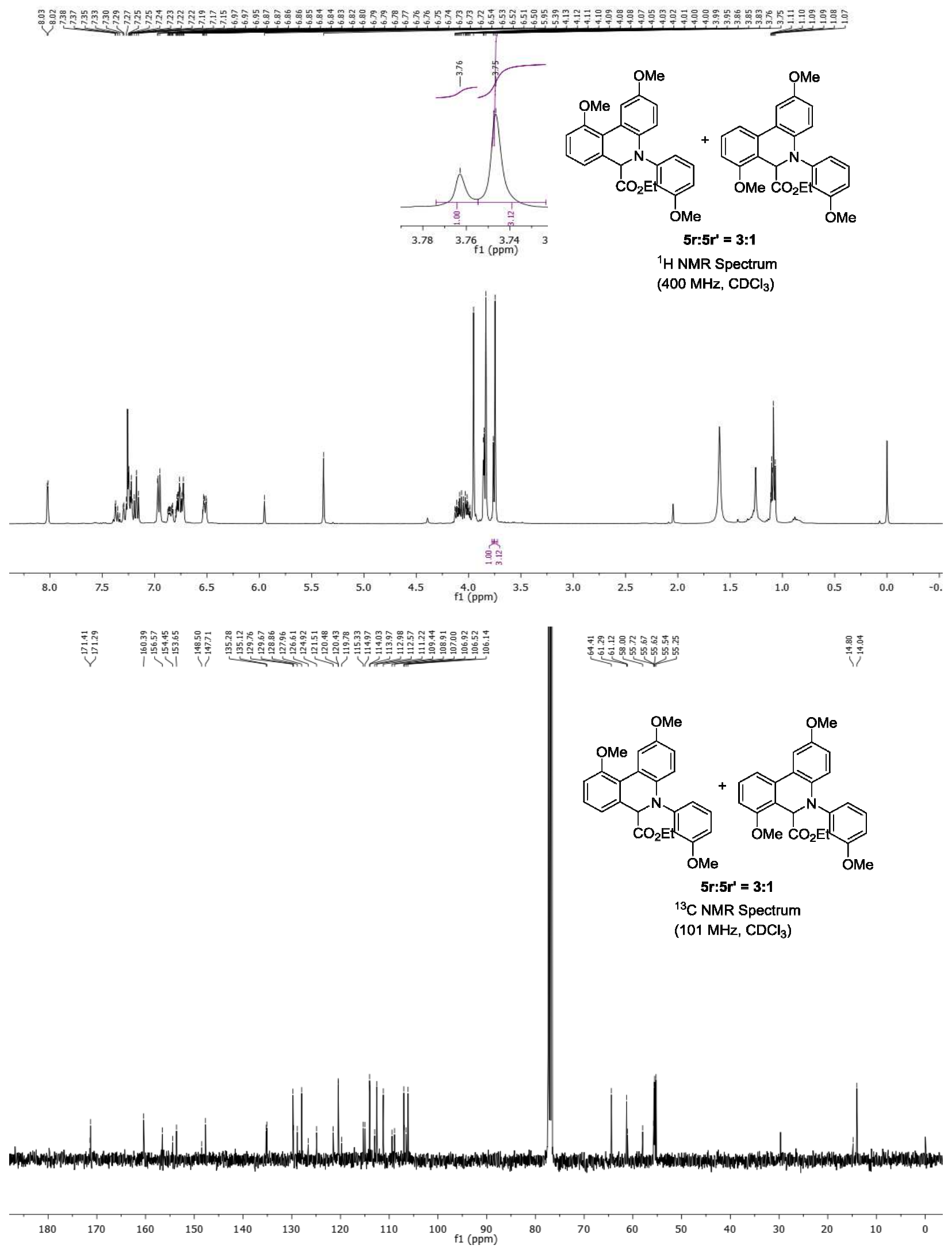


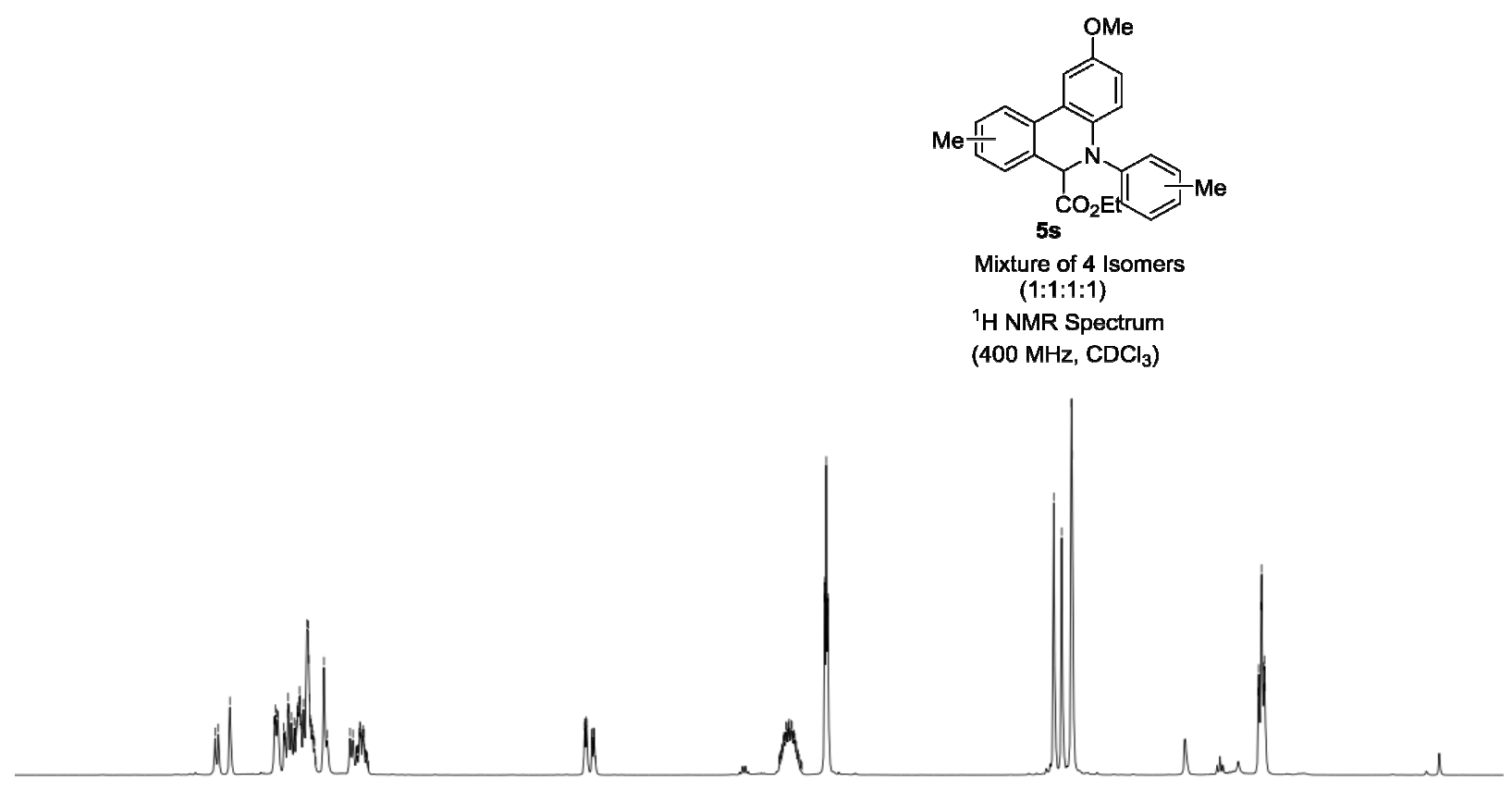

\begin{tabular}{|c|c|c|c|c|c|c|c|c|c|c|c|c|c|c|c|c|}
\hline 8.5 & 8.0 & 7.5 & 7.0 & 6.5 & 6.0 & 5.5 & 5.0 & ${ }_{\mathrm{f} 1}^{4.5}(\mathrm{ppm})^{4.0}$ & 3.5 & 3.0 & 2.5 & 2.0 & 1.5 & 1.0 & 0.5 & 0.0 \\
\hline
\end{tabular}

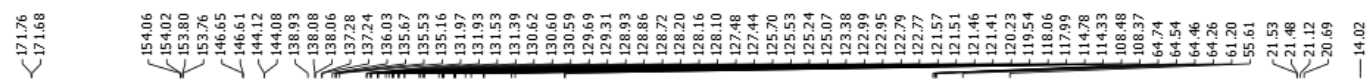

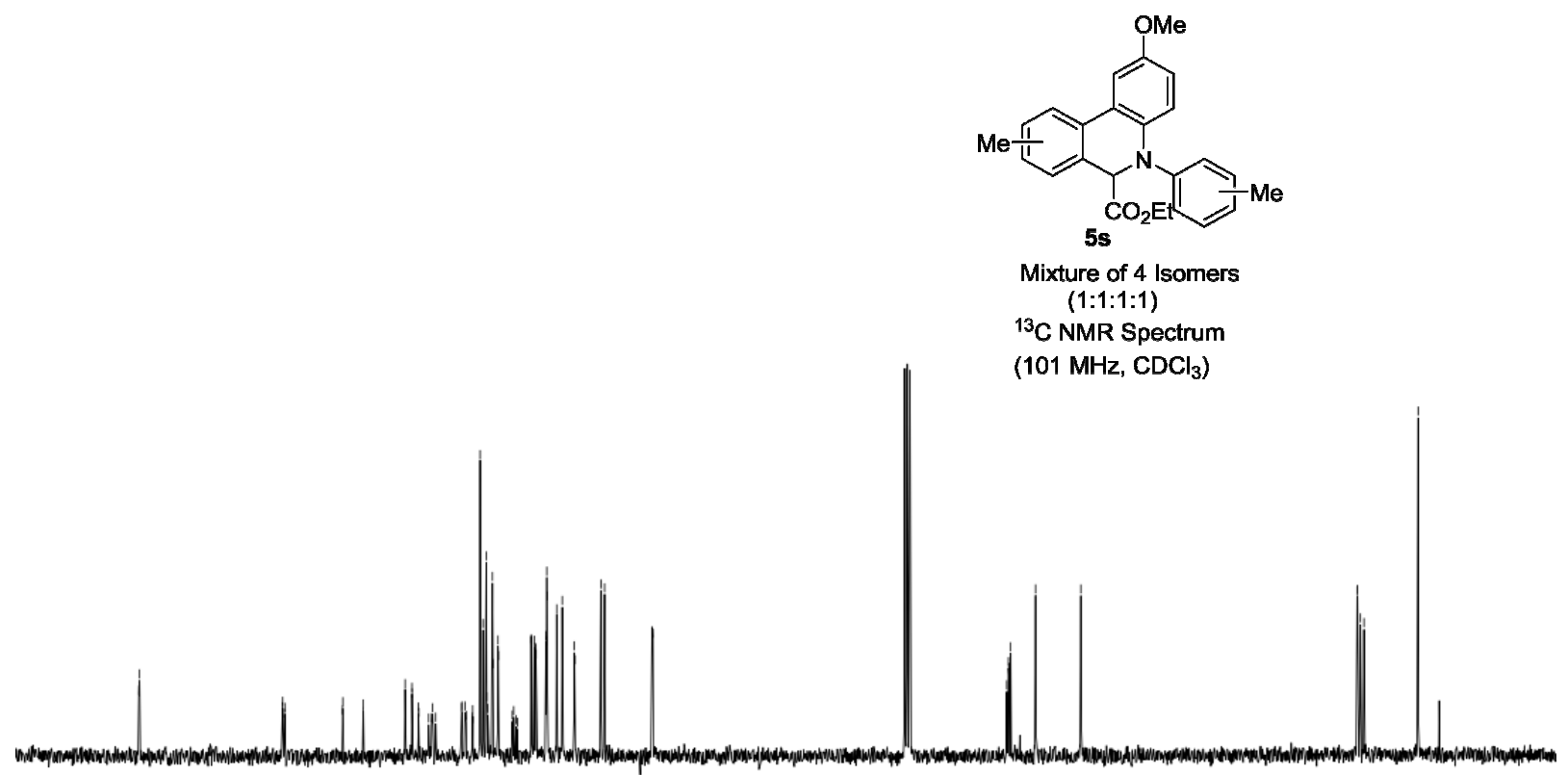

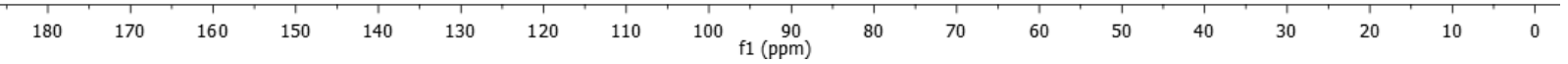



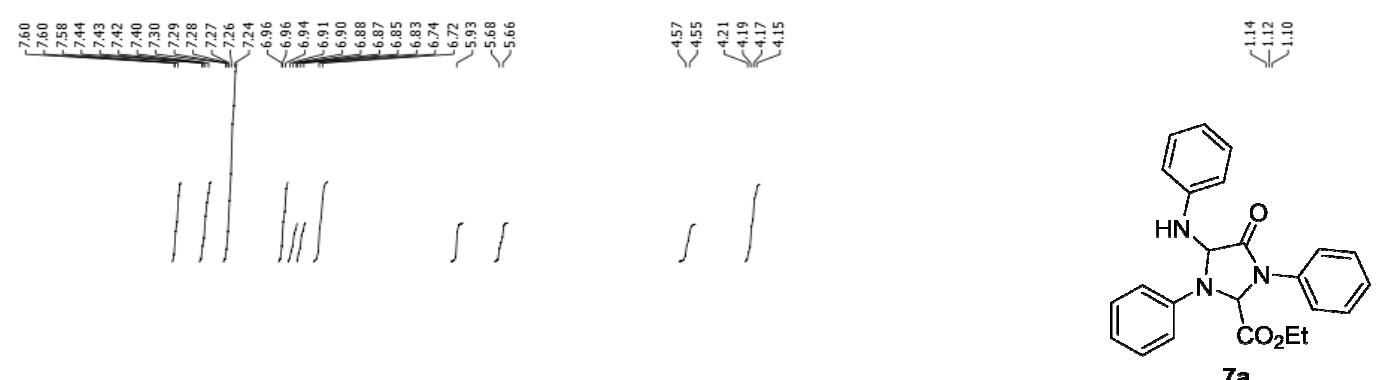

${ }^{1} \mathrm{H} \mathrm{NMR} \mathrm{Spectrum}^{3}$ $\left(400 \mathrm{MHz}, \mathrm{CDCl}_{3}\right.$ )
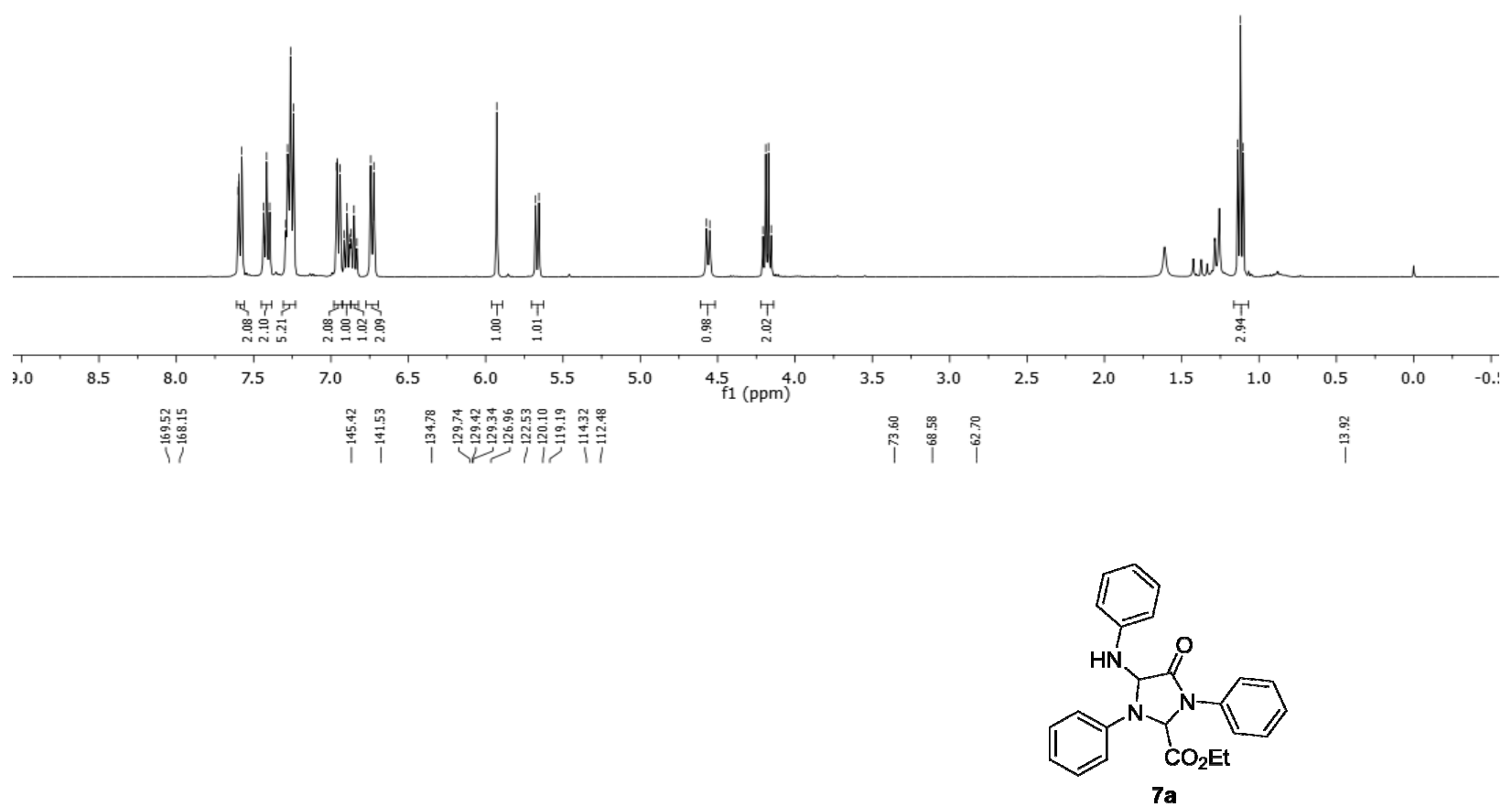

${ }^{13} \mathrm{C}$ NMR Spectrum ${ }^{3}$ (101 MHz, $\mathrm{CDCl}_{3}$ )
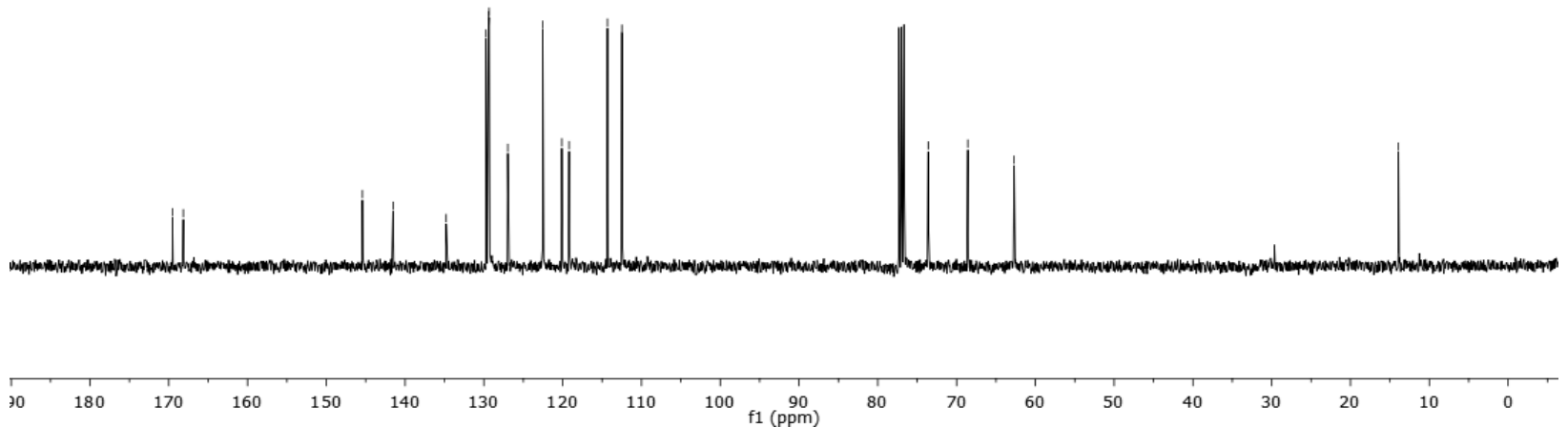

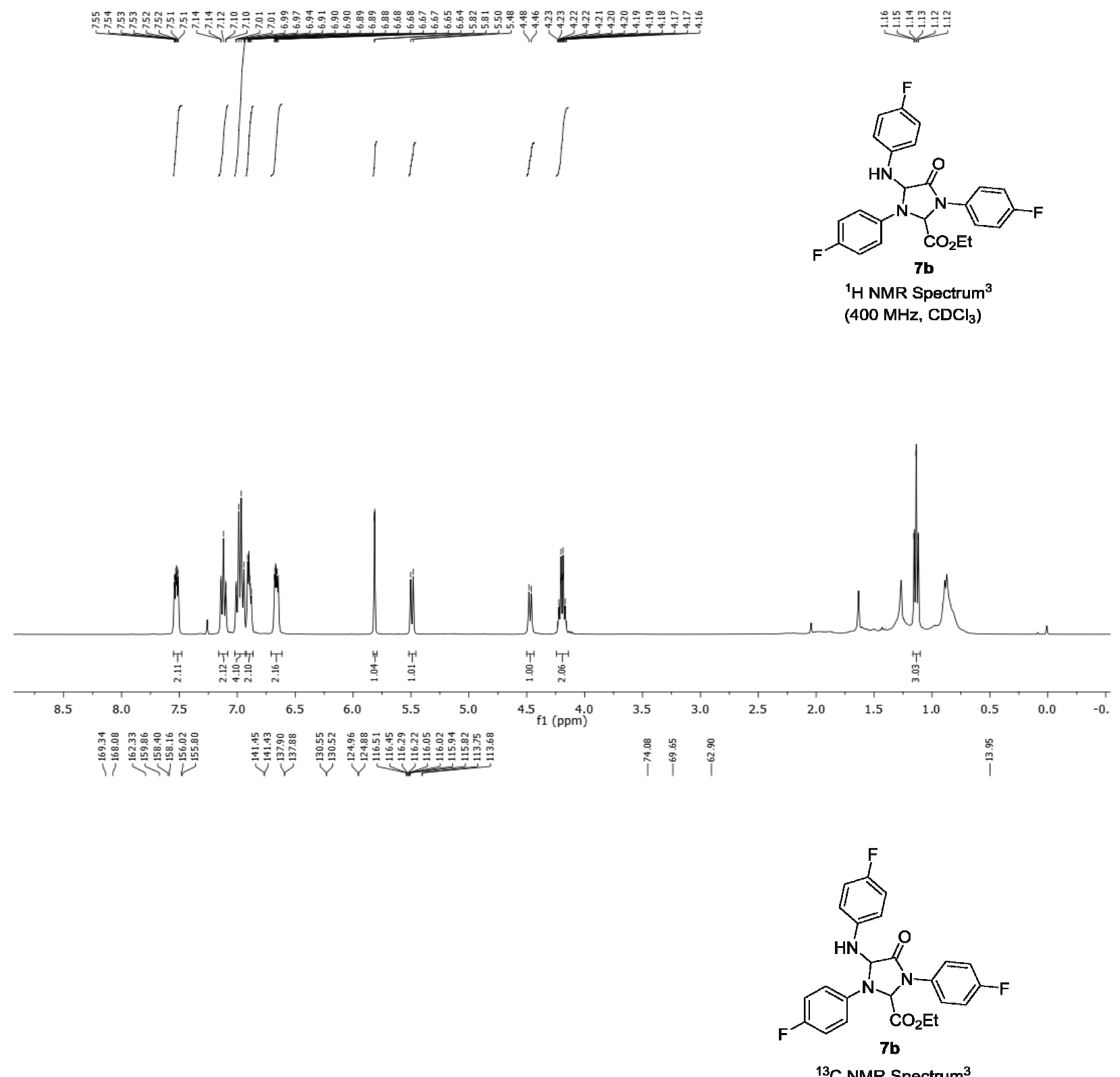

NMR Spectrum (101 MHz, $\mathrm{CDCl}_{3}$ )

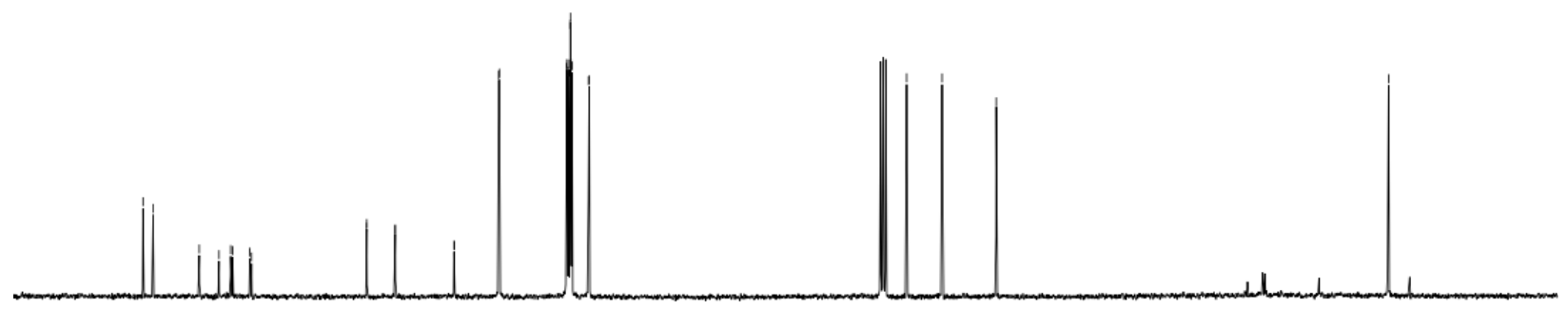



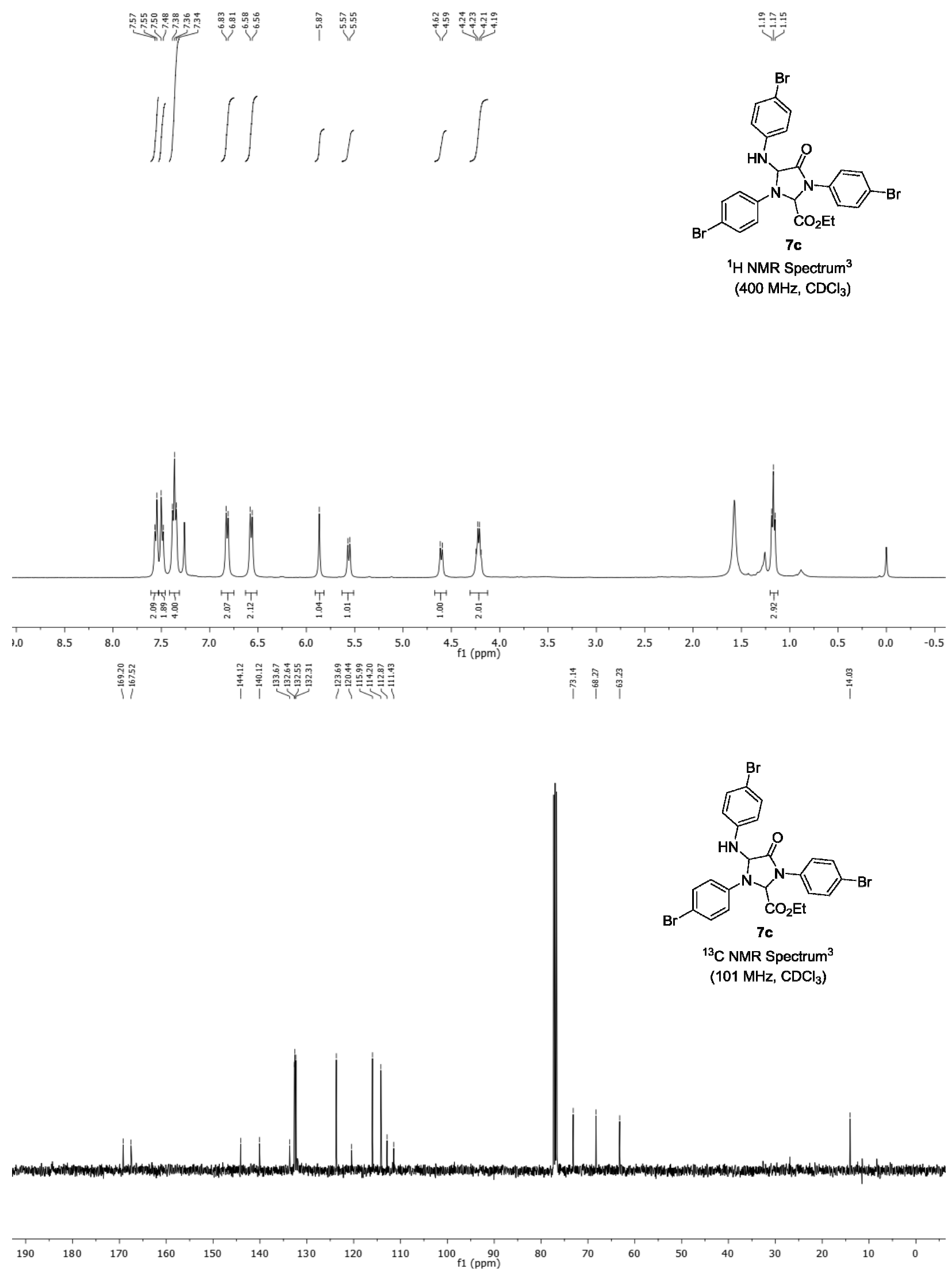

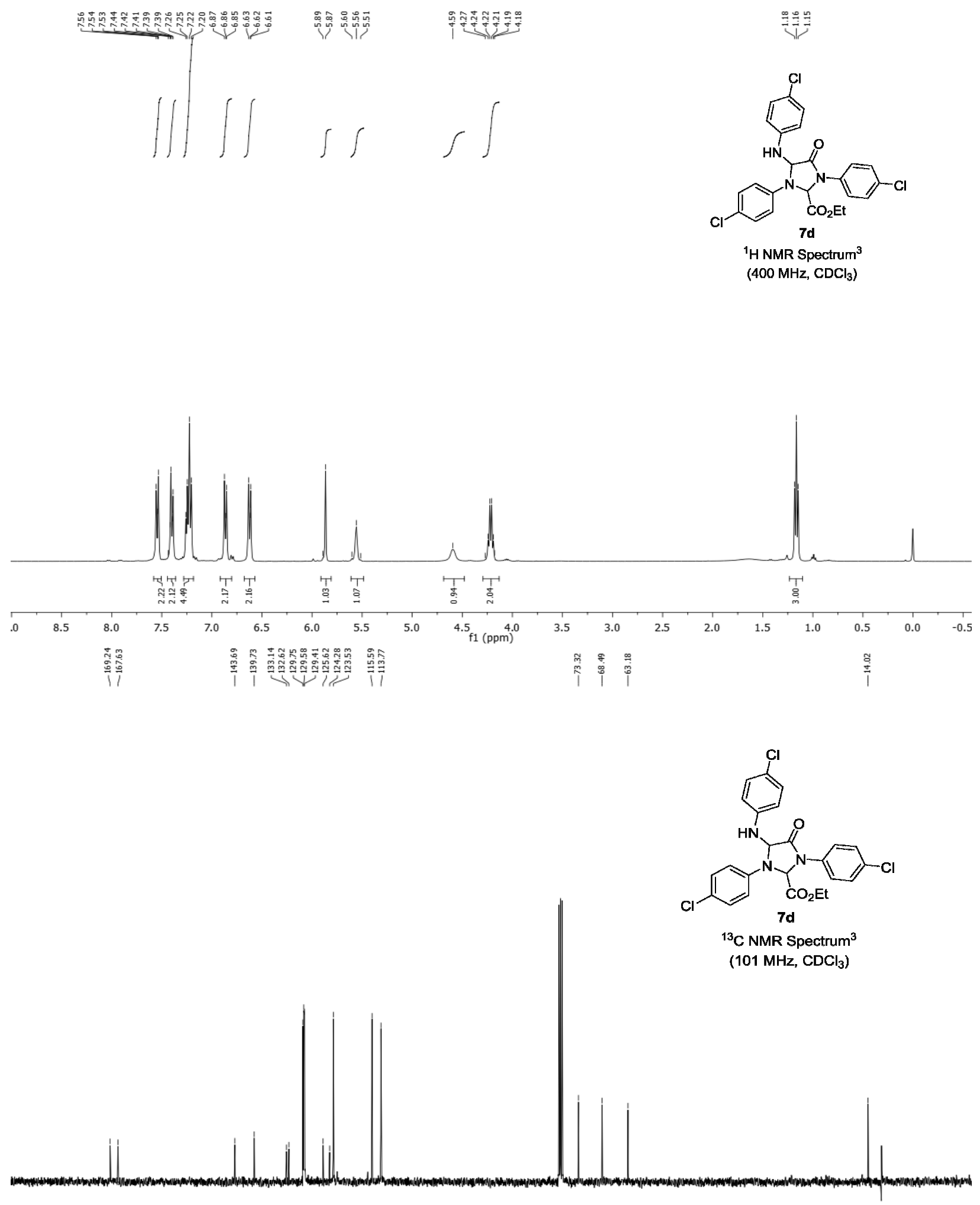

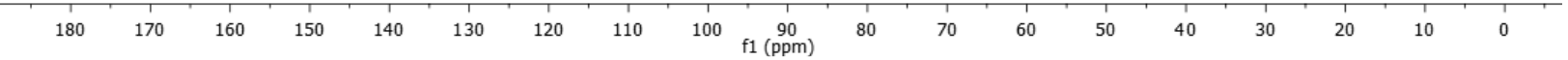



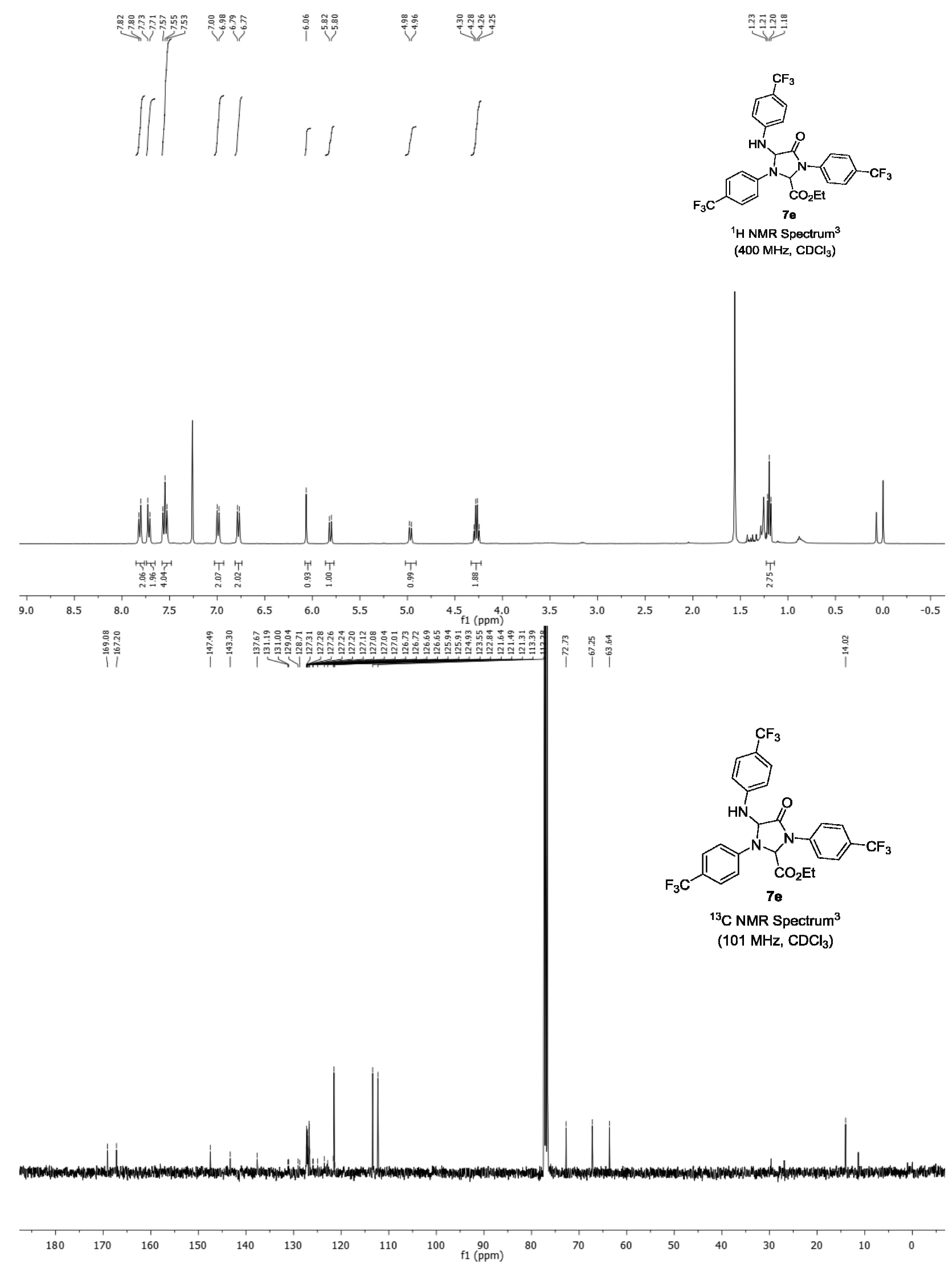

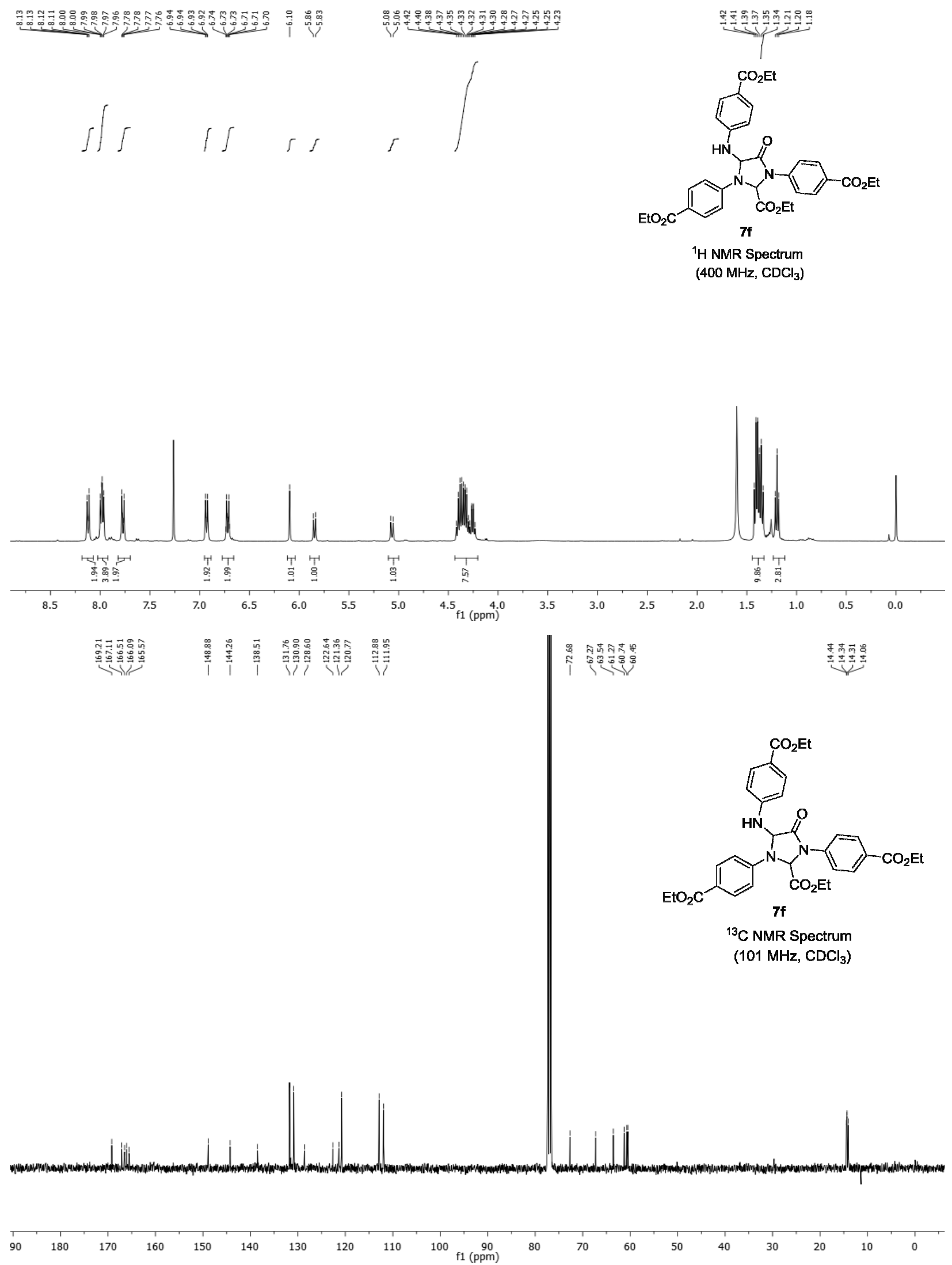

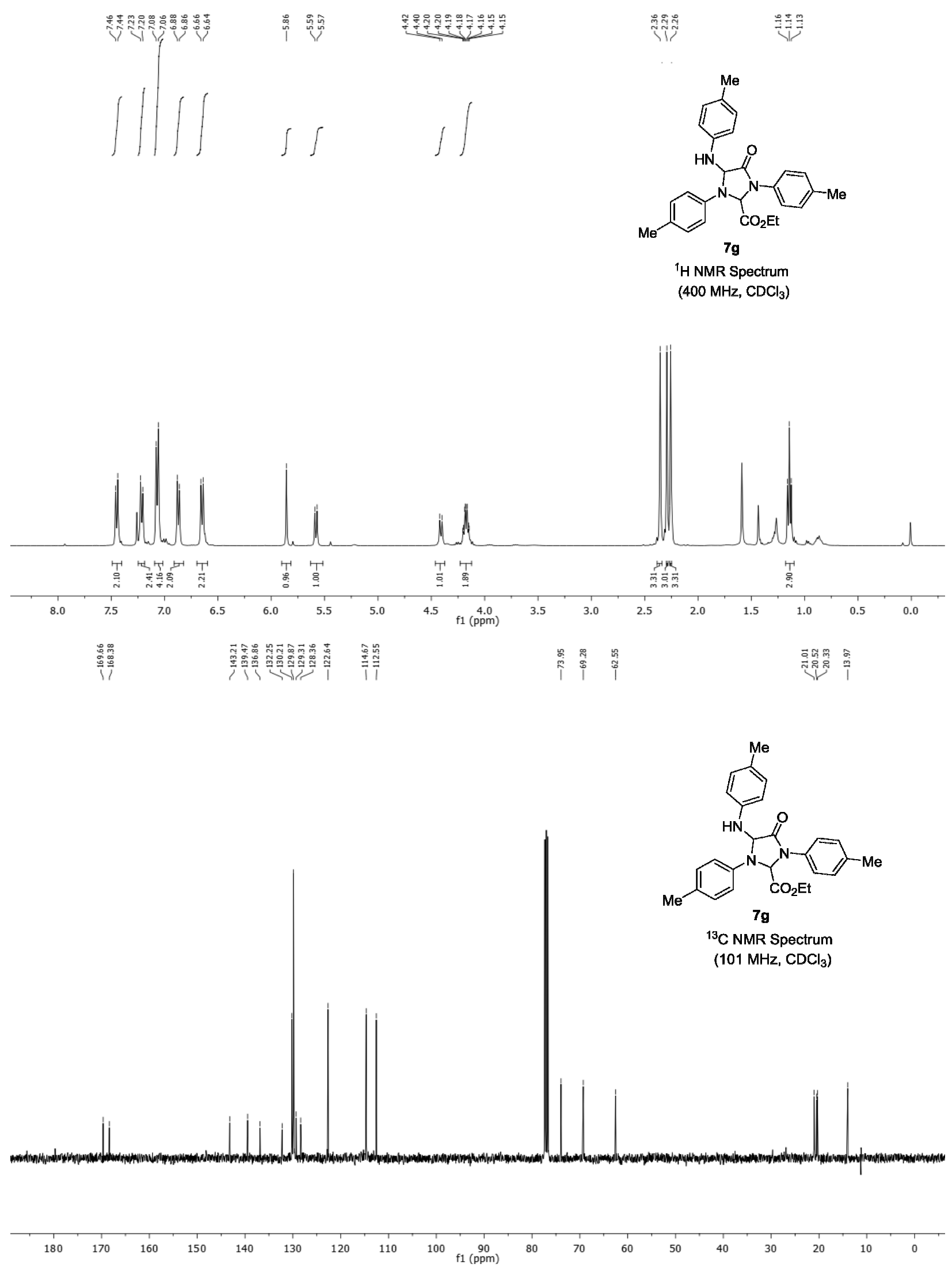


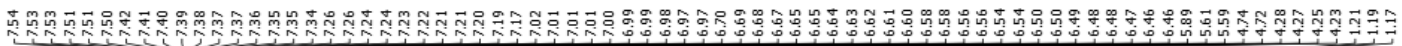
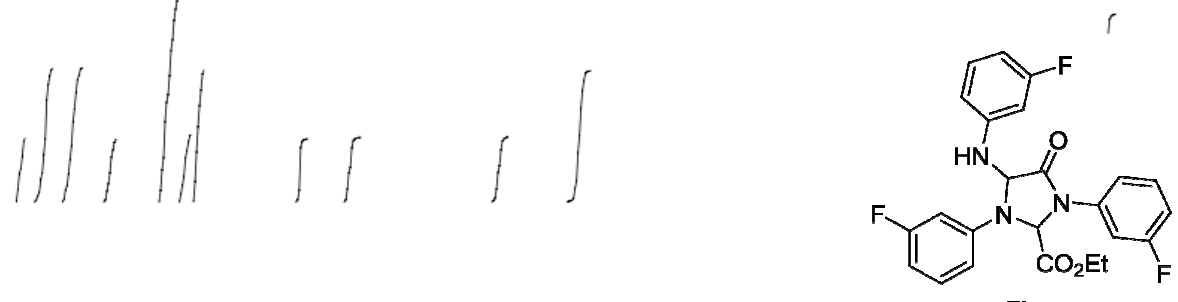

$7 i$

${ }^{1} \mathrm{H}$ NMR Spectrum ${ }^{3}$

(400 $\mathrm{MHz}, \mathrm{CDCl}_{3}$ )
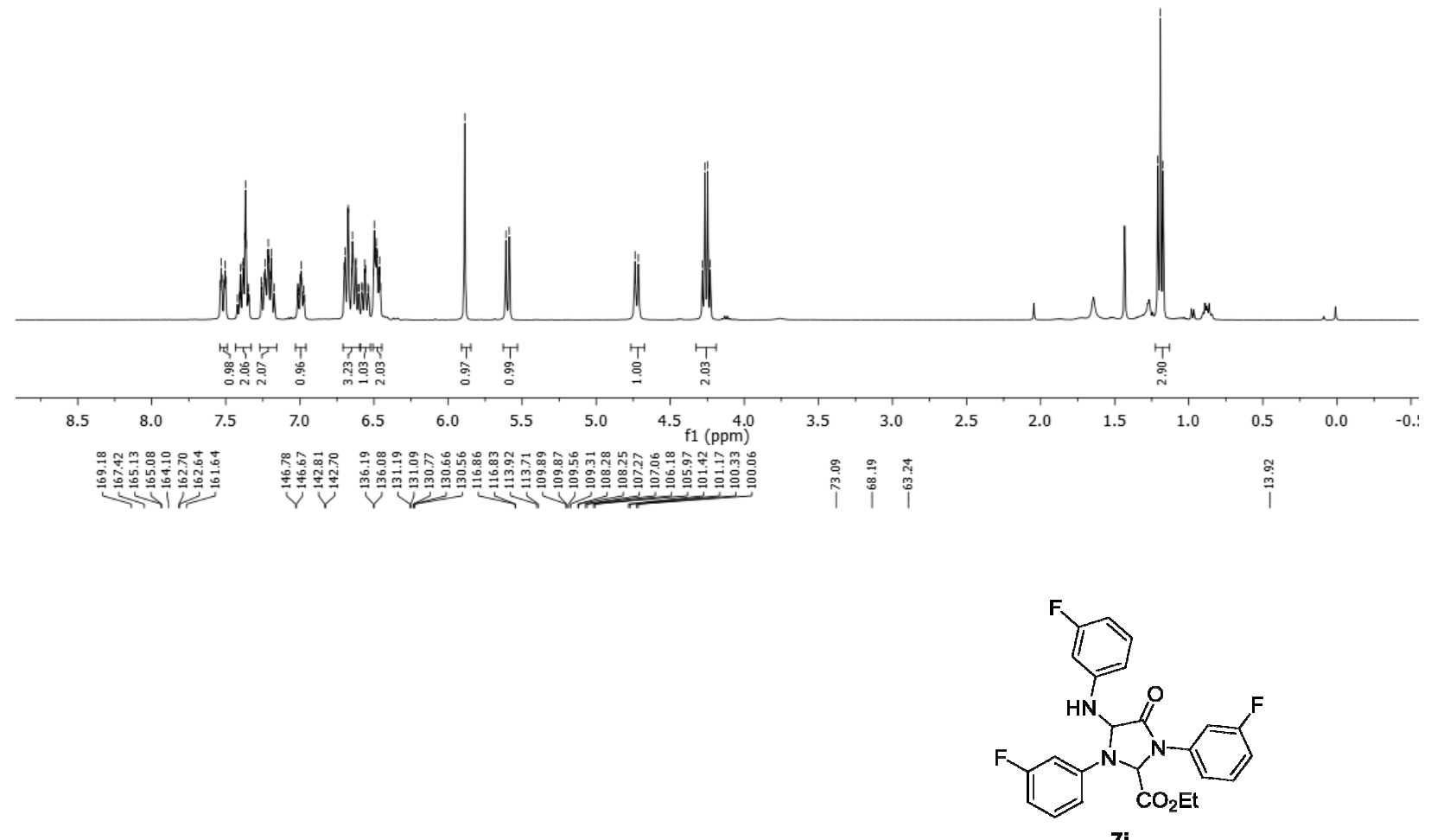

${ }^{13} \mathrm{C}$ NMR Spectrum ${ }^{3}$ (101 MHz, $\mathrm{CDCl}_{3}$ )
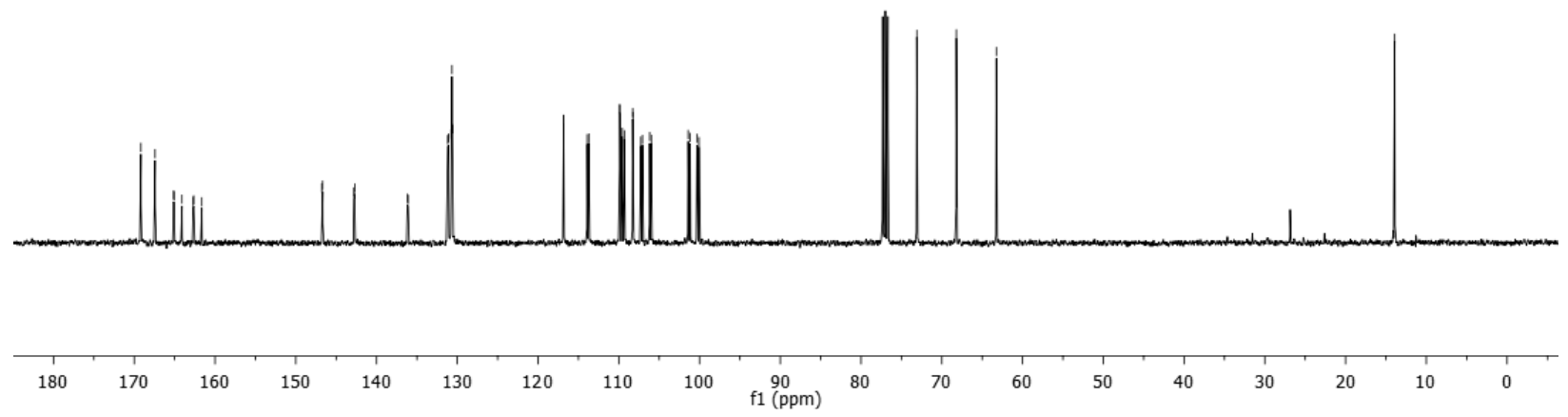

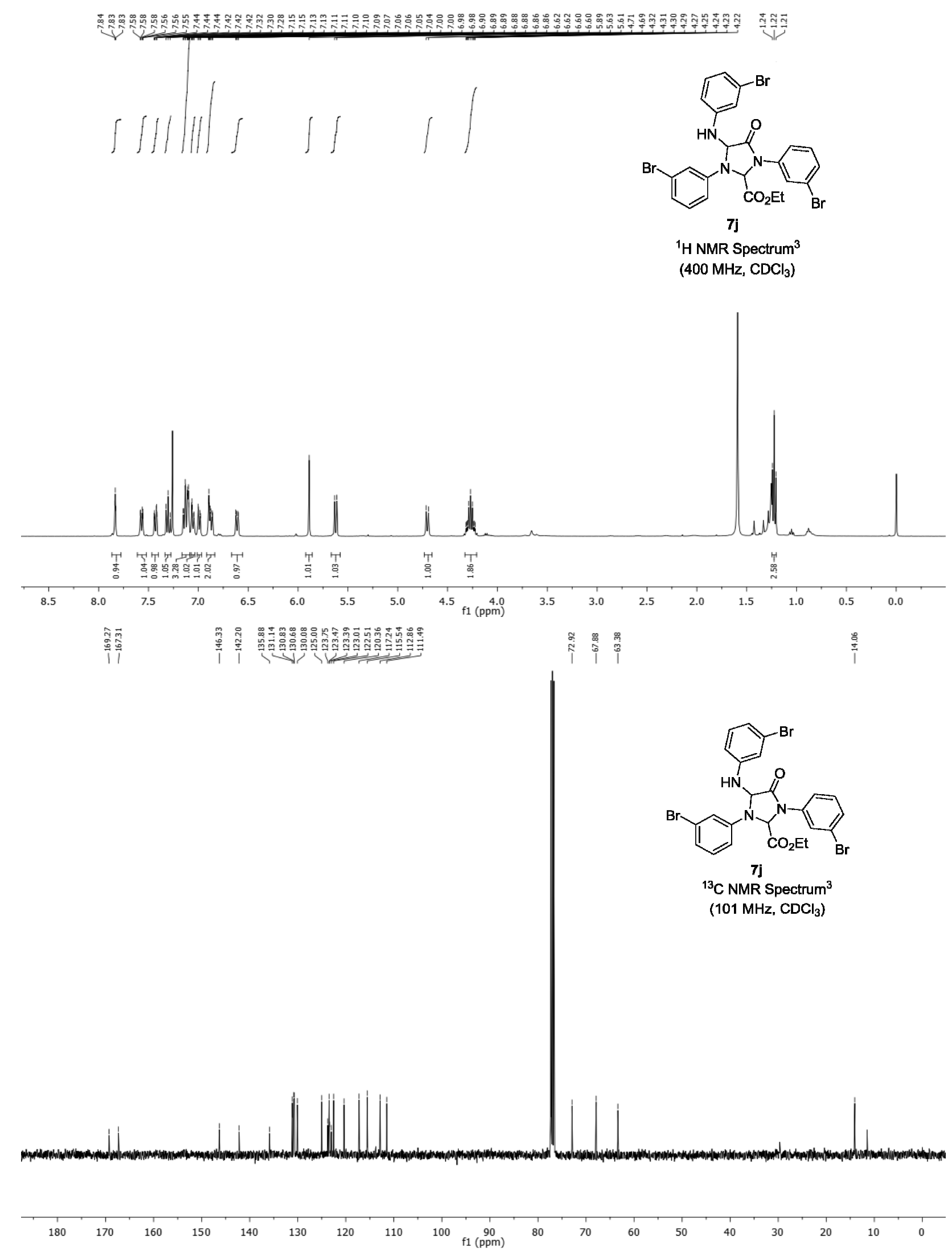

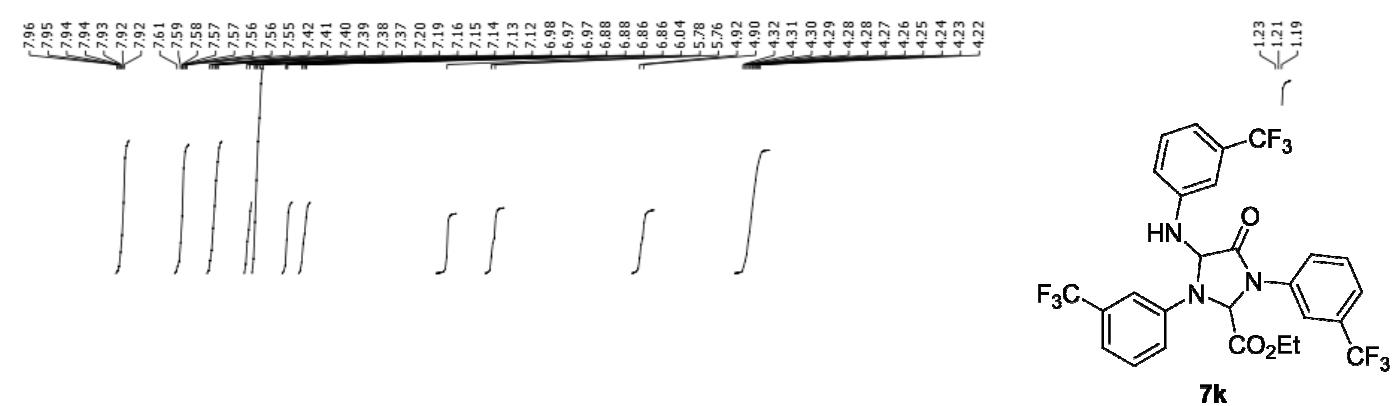

${ }^{1} \mathrm{H}$ NMR Spectrum ${ }^{3}$ $\left(400 \mathrm{MHz}, \mathrm{CDCl}_{3}\right.$ )
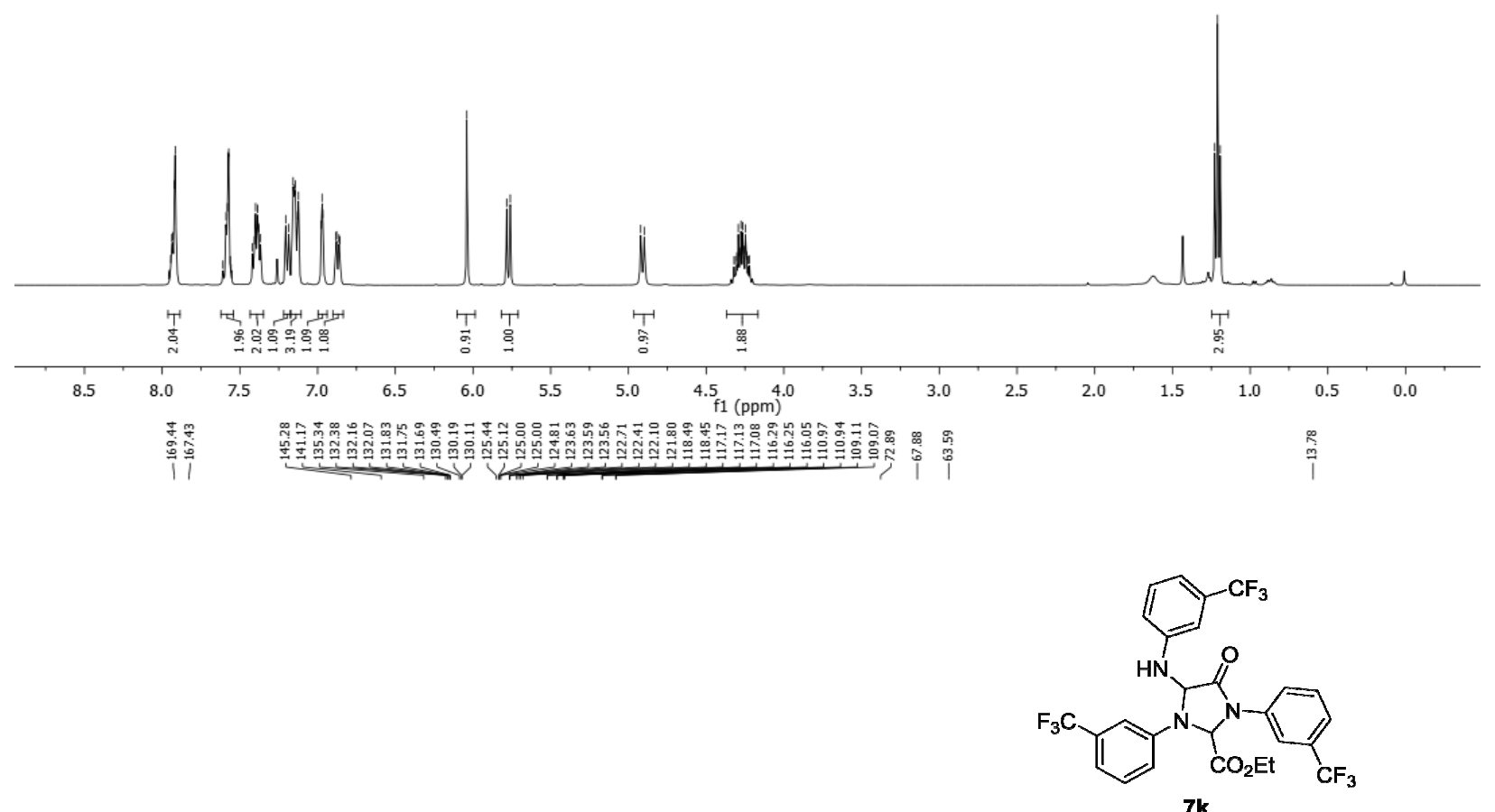

$7 k$

${ }^{13} \mathrm{C}$ NMR Spectrum ${ }^{3}$ (101 MHz, $\mathrm{CDCl}_{3}$ )

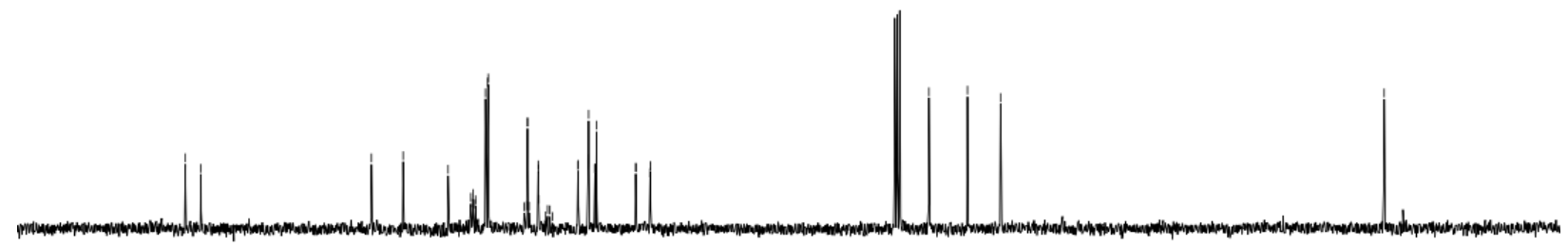

.90

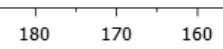

150

140

110

$00 \begin{gathered}90 \\ \mathrm{f} 1(\mathrm{ppm})\end{gathered}$

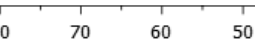

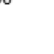

